\title{
Single Ion Trapping for the Enriched Xenon Observatory
}

\author{
Samuel J. Waldman \\ Stanford Linear Accelerator Center \\ Stanford University \\ Stanford, CA 94309
}

SLAC-Report-813

Prepared for the Department of Energy

under contract number DE-AC02-76SF00515

Printed in the United States of America. Available from the National Technical Information Service, U.S. Department of Commerce, 5285 Port Royal Road, Springfield, VA 22161. 
This document, and the material and data contained therein, was developed under sponsorship of the United States Government. Neither the United States nor the Department of Energy, nor the Leland Stanford Junior University, nor their employees, nor their respective contractors, subcontractors, or their employees, makes an warranty, express or implied, or assumes any liability of responsibility for accuracy, completeness or usefulness of any information, apparatus, product or process disclosed, or represents that its use will not infringe privately owned rights. Mention of any product, its manufacturer, or suppliers shall not, nor is it intended to, imply approval, disapproval, or fitness of any particular use. A royalty-free, nonexclusive right to use and disseminate same of whatsoever, is expressly reserved to the United States and the University. 


\title{
SINGLE ION TRAPPING FOR THE ENRICHED XENON OBSERVATORY
}

\author{
A DISSERTATION \\ SUBMITTED TO THE DEPARTMENT OF APPLIED PHYSICS \\ AND THE COMMITTEE ON GRADUATE STUDIES \\ OF STANFORD UNIVERSITY \\ IN PARTIAL FULFILLMENT OF THE REQUIREMENTS \\ FOR THE DEGREE OF \\ DOCTOR OF PHILOSOPHY
}

Samuel J. Waldman

May 2005 
(c) Copyright by Samuel J. Waldman 2005

All Rights Reserved 


\section{Abstract}

In the last decade, a variety of neutrino oscillation experiments have established that there is a mass difference between neutrino flavors, without determining the absolute neutrino mass scale. The Enriched Xenon Observatory for neutrinoless double beta decay (EXO) will search for the rare decays of xenon to determine the absolute value of the neutrino mass. The experiment uses a novel technique to minimize backgrounds, identifying the decay daughter product in real time using single ion spectroscopy. Here, we describe single ion trapping and spectroscopy compatible with the EXO detector. We extend the technique of single ion trapping in ultrahigh vacuum to trapping in xenon gas. With this technique, EXO will achieve a neutrino mass sensitivity of $\left\langle m_{\nu}\right\rangle \simeq .010 \mathrm{eV}$. 


\section{Acknowledgements}

To my friends, family, and co-workers: thank you.

To the members of Rains 14J, the Casa de Alameda de Las Pulgas, and the Institute of Dudes Dudes Dudes (IDDD): you made life outside lab worth living;

To Jason Detwiler and Nikolai Tolich, Gratta Group class of '05: you made life inside lab worth living;

To Jesse Wodin, Matt Green, Thomas Koffas, and everyone else on EXO: thank you for making EXO the best physics experiment in the world;

To Prof. Mara Prentiss and Prof. Giorgio Gratta: you have been my mentors in physics - I hope I live up to your example;

and to my family, Mom, Dad, David and Caolionn: you have been my mentors in life - without you, none of this is possible. 


\section{Contents}

Abstract $\quad$ v

Acknowledgements vii

1 Introduction 1

2 Massive neutrinos $\quad 5$

2.1 Double beta decay . . . . . . . . . . . . . . . . 6

2.2 Present status of $0 \nu \beta \beta$ searches . . . . . . . . . . . . . . 12

2.3 EXO detector design concepts . . . . . . . . . . . . . . 15

2.4 Liquid TPC concept . . . . . . . . . . . . . . . . . . . . . 17

2.5 Expected detector performance . . . . . . . . . . . . . . . 18

3 Laser Induced Fluorescence $\quad 23$

3.1 Ba-II spectroscopy . . . . . . . . . . . . . . . . . 24

3.2 Two level systems . . . . . . . . . . . . . . . . . . . . . . . 24

3.3 Collisonal line broadening . . . . . . . . . . . . . . . . . 27

3.4 Doppler broadening . . . . . . . . . . . . . . . . . . 31

3.5 Optical Bloch Equations . . . . . . . . . . . . . . . . 34

4 The theory of single ion trapping 41

4.1 Ideal traps . . . . . . . . . . . . . . . . . . . . . 41

4.2 The adiabatic approximation . . . . . . . . . . . . . . 47

4.3 Non-ideal traps . . . . . . . . . . . . . . . . . . . . . . . . . . . . . . 49 
5 Trapping with buffer gas $\quad 55$

5.1 Collisions in the literature . . . . . . . . . . . . . . 56

5.2 Ion-ion collisions . . . . . . . . . . . . . . . . . . . . . . . 59

5.3 Brownian motion . . . . . . . . . . . . . . . . 61

5.4 Ion-neutral collisions . . . . . . . . . . . . . . . . . . . . 62

5.4.1 Scattering kinematics . . . . . . . . . . . 63

5.4 .2 Induced dipole interactions . . . . . . . . . . . . . . . . 64

5.5 Scattering in phase space . . . . . . . . . . . . . . 67

6 Experimental results $\quad 71$

6.1 Basic experimental setup . . . . . . . . . . . . . . . 71

6.2 Hyperbolic trap in vacuum . . . . . . . . . . . . . . . . 76

6.2.1 Signal quantization . . . . . . . . . . . . . 76

6.2.2 Ion fluorescence spectrum . . . . . . . . . . . . . . 77

6.3 Trap asymmetry and RF micromotion . . . . . . . . . . . . . . . . 79

6.4 Hyperbolic trap in gas . . . . . . . . . . . . . . . . . . . 83

6.4.1 Buffer gas filling. . . . . . . . . . . . . . 83

6.4.2 Spatial distribution . . . . . . . . . . . . . . . . 84

6.4.3 Buffer gas spectra . . . . . . . . . . . . 87

6.5 Argon buffer gas . . . . . . . . . . . . . . . . . . 90

6.6 Heavy buffer gases . . . . . . . . . . . . . . . . . . . . 91

6.6.1 Krypton unloading .................. 93

6.6.2 Pressure dependence . . . . . . . . . . . . . . . . . . . . 94

6.6.3 RF dependence . . . . . . . . . . . . . . . . . 96

6.6.4 Ion pump filter . . . . . . . . . . . . . . . . . . . 98

6.7 Unloading mechanism . . . . . . . . . . . . . . . . . . . . . 99

7 Diffusion unloading simulation 103

7.1 Simulation configuration . . . . . . . . . . . . . 103

7.2 Comparison to theory . . . . . . . . . . . . . . . . . . . . 104

7.3 Trapped ion diffusion . . . . . . . . . . . . . . . . . 106

7.4 Simulated buffer gas spectra . . . . . . . . . . . . . . 108 
7.4.1 RF independent velocity distribution . . . . . . . . . 108

7.4.2 RF dependent signal amplitude . . . . . . . . . . . . . . 109

7.4.3 Pressure dependent velocity spectra . . . . . . . . . . . . 111

7.5 Simulated decay time constant . . . . . . . . . . . . . . . 113

7.5.1 Temperature dependent unloading . . . . . . . . . . . . 117

8 Conclusion $\quad 119$

$\begin{array}{ll}\text { A Experimental Setup } & 121\end{array}$

A.1 Frequency stabilized laser systems . . . . . . . . . . . . . . 121

A.1.1 External cavity diode lasers . . . . . . . . . . . . . 122

A.1.2 Second harmonic generation . . . . . . . . . . . . . 124

A.1.3 Frequency stabilization . . . . . . . . . . . . . . . . . 124

A.2 Vacuum and gas handling . . . . . . . . . . . 126

A.3 RF quadrupole trap electronics . . . . . . . . . . . . . 128

$\begin{array}{ll}\text { Bibliography } & 131\end{array}$ 


\section{List of Tables}

2.1 Phase space factors for $0 \nu \beta \beta$ decay . . . . . . . . . . . . . . . . . . . 12

$2.20 \nu \beta \beta$ half-life sensitivities . . . . . . . . . . . . . . . . . . . . 13

2.3 Predicted sensitivity to $0 \nu \beta \beta$. . . . . . . . . . . . . . . . 21

3.1 Linewidth contributions . . . . . . . . . . . . . . . 35

4.1 Parameters used with $5 \mathrm{~mm}$ hyperbolic trap . . . . . . . . . . . 50

5.1 Thermal trapping volume . . . . . . . . . . . . . . . 61

5.2 Table of mean free times . . . . . . . . . . . . . . . . . . 67 


\section{List of Figures}

$2.1 \quad$ Atomic mass scheme for $\mathrm{A}=136 \ldots \ldots \ldots \ldots \ldots$

2.2 Double beta decay Feynman diagrams f . . . . . . . . . . . 8

2.3 Majorana mass dependence on the absolute neutrino mass . . . . . 9

2.4 Double beta decay modes . . . . . . . . . . . . . . . . 10

2.5 The Gotthard Xe TPC . . . . . . . . . . . . . . . . . . . . 15

2.6 Conceptual liquid xenon $\mathrm{TPC} \ldots \ldots \ldots \ldots$

3.1 Barium ion levels . . . . . . . . . . . . . . . . . . . . . . . 24

3.2 Two level system . . . . . . . . . . . . . . . . . . . . . . 25

3.3 Lorentzian function . . . . . . . . . . . . . . . . . . . 28

3.4 Interatomic collisions . . . . . . . . . . . . . . . . . . . . . 29

3.5 Lorentzian and Gaussian . . . . . . . . . . . . . . . . . 33

3.6 Modulated absorption spectrum . . . . . . . . . . . . . . 34

3.7 Optical Bloch Equations . . . . . . . . . . . . . . . . . . . . . 39

4.1 Hyperbolic trap . . . . . . . . . . . . . . . . . . . . . . . . 42

4.2 Single ion stability plot . . . . . . . . . . . . . . . . 44

4.3 Trapped ion motion . . . . . . . . . . . . . . . . . 46

4.4 Fourier transform of ion motion . . . . . . . . . . . . . 46

5.1 Trajectories in an induced dipole potential . . . . . . . . 66

6.1 Basic experiment setup . . . . . . . . . . . . . . . . . . . . 72

6.2 Microscope view of the trap . . . . . . . . . . . . . . . 72

6.3 RF electronics schematic . . . . . . . . . . . . . . . . . . 73 
6.4 Millikan "ion drop" experiment . . . . . . . . . . . . . . . . . . 78

6.5 UHV resonance spectrum . . . . . . . . . . . . . . . . 80

6.6 Manifestations of trap asymmetry . . . . . . . . . . . . . 82

6.7 Helium buffer spatial distribution . . . . . . . . . . . . . . 85

6.8 Helium buffer gas fluorescence signal . . . . . . . . . . . . . . 86

6.9 Helium buffer gas (II) . . . . . . . . . . . . . . . . . . . . . . 87

6.10 Pressure dependent helium spectra . . . . . . . . . . . . . . 88

6.11 Voight spectrum in helium . . . . . . . . . . . . . . . . 89

6.12 Spectra width vs. RF voltage . . . . . . . . . . . . . . 89

6.13 Argon doppler spectra . . . . . . . . . . . . . . . . . . . . 91

6.14 Xenon unloading I . . . . . . . . . . . . . . . . . . . . . 92

6.15 Xenon unloading II . . . . . . . . . . . . . . . . . 93

6.16 Krypton unloading . . . . . . . . . . . . . . . . 94

6.17 Xenon step unloading . . . . . . . . . . . . . . . 95

6.18 Xenon step unloading in helium . . . . . . . . . . . . . . . . . . 97

6.19 Unloading RF dependence . . . . . . . . . . . . . . . . . . . 98

7.1 Simulated trapped ion behaviour in buffer gases . . . . . . . . . 105

7.2 Simulated time series in helium buffer . . . . . . . . . . . . 106

7.3 Simulated time series in xenon buffer . . . . . . . . . . . . 107

7.4 Histogram of envelope . . . . . . . . . . . . . . . . . 107

7.5 Simulated velocity spectra vs. RF voltage . . . . . . . . . . . 109

7.6 Velocity $1 / e$ width vs. RF voltage . . . . . . . . . . . . . . 110

7.7 Ion radius vs. RF voltage . . . . . . . . . . . . . . . . . . 110

7.8 Simulated fluorescence vs. RF voltage . . . . . . . . . . . . . . 111

7.9 Simulated velocity vs. pressure . . . . . . . . . . . . . . 112

7.10 Simulated fading time constants in xenon . . . . . . . . . . . . . 114

7.11 Simulated time constant pressure dependence . . . . . . . . . . . . 115

7.12 Simulated spatial distribution vs. buffer gas temperature . . . . . . . 117

A.1 External cavity diode laser schematic . . . . . . . . . . . . . . 122

A.2 External cavity diode laser gain curve . . . . . . . . . . . . . 123 
A.3 Second harmonic generation . . . . . . . . . . . . . . . 125

A.4 Vacuum and gas systems . . . . . . . . . . . . . . . . 127

A.5 RF electronics . . . . . . . . . . . . . . . . . . . 129 
xviii 


\section{Chapter 1}

\section{Introduction}

In the last ten years, the results from a variety of experiments have revolutionized neutrino physics. The combined evidence from SuperK [1], SNO [2], KamLAND [3] and other neutrino oscillation experiments indicates that neutrinos have finite mass. This discovery offers exciting possibilities to explore physics beyond the standard model, particularly by measuring the absolute value of the neutrino mass which cannot be determined by neutrino oscillation measurements.

The Enriched Xenon Observatory for neutrinoless double beta decay, EXO, will directly measure the neutrino mass by observing rare decays of ${ }^{136} \mathrm{Xe}$. In the standard model version of double beta decay, a nucleus decays by emitting two electrons and two neutrinos. In the neutrino-less version of double beta decay, the nucleus decays with the emission of two electrons and no neutrinos; a single neutrino participates in both weak vertices. If observed, neutrinoless double beta decay $(0 \nu \beta \beta)$ would have profound implications for many area of physics ranging from cosmology and astrophysics to particle physics and string theory.

Two conditions must be satisfied for $0 \nu \beta \beta$ to be occur: the neutrino must have a finite mass and the neutrino must be its own anti-particle. If these conditions are met, the rate at which an atom decays via $0 \nu \beta \beta$ depends on the mass of the neutrino. However, because double beta decay is a second order weak process, the decay mode has a very long half-life. Due to the low rate, double beta decay experiments must have extremely low backgrounds; for example, the current best limit on a $0 \nu \beta \beta$ decay 
half-life is $T_{1 / 2}>1.9 \times 10^{25}$ yrs, measured for ${ }^{76} \mathrm{Ge}$ [4]. Traditional double beta decay experiments rely on electron calorimetry and tracking to determine the decay rate, and future experiments are no different. In most proposed experiments, the backgrounds are minimized by using a source material that also functions as the electron calorimeter, by employing ultra-low background techniques to minimize backgrounds from radioactivity, and by using a detector with high energy resolution. In order to improve the neutrino mass sensitivity, future experiments must substantially increase the source mass by one to two orders of magnitude over past experiments while reducing backgrounds. To maintain a reasonable detector size, a high density source must be used, reducing the effectiveness of electron tracking in eliminating backgrounds.

The EXO detector uses a large xenon Time Projection Chamber (TPC) as the best solution to increase detector mass, to increase the energy resolution and to reduce backgrounds. In the TPC, ${ }^{136} \mathrm{Xe}$ serves as the double beta decay source and the electron calorimeter, combining very low background with high energy resolution. This detector design has a large fiducial volume free from all materials except the source xenon, inherently reducing radioactive backgrounds by isolating the active volume from outside influence.

Unique among proposed double beta decay experiments, EXO detects the double beta decay daughter atom in quasi-real time using single ion laser spectroscopy. The use of single ion spectroscopy for background reduction was first proposed by M.K. Moe in 1991 [5] who observed that ${ }^{136} \mathrm{Xe}$ double beta decays to ${ }^{136} \mathrm{Ba}$, and barium ions (Ba-II) have been studied in single ion trapping experiments for more than two decades [6]. In the proposed EXO design, ions are extracted from candidate decay sites in a large xenon TPC and taken to a spectroscopic cell for analysis. Using this technique, EXO will have a background free neutrino mass sensitivity of $<0.010 \mathrm{eV}$ using up to 10 tons of isotopically enriched ${ }^{136} \mathrm{Xe}$. We discuss the EXO experiment in detail in the following chapter.

This thesis represents the first study of Ba-II spectroscopy for use in the detection of double beta decay. The majority of this thesis is concerned with the trapping and spectroscopy of barium ions in high pressure buffer gas. Specifically, we examine the dynamics of barium trapping in xenon for use with the EXO detector. We find 
that barium can only be trapped with low xenon partial pressures due to the effects of diffusion unloading. This phenomenon, poorly studied prior to this work, is a general mechanism of ion trap unloading as a result of collisions between trapped ions and heavy buffer gases. After describing the EXO experiment in Chapter 2, we discuss Ba-II spectroscopy in Chapter 3, ion trapping in Chapter 4, and buffer gas collisions in Chapter 5. Chapter 6 details the results of experiments with Ba-II in a variety of buffer gases from helium to xenon, including the phenomenology of diffusion unloading. Chapter 7 presents results of simulations which qualitatively describe the experimental data. We conclude with implications for the EXO experimental program in Chapter 8. 


\section{Chapter 2}

\section{Massive neutrinos}

Despite the increasing precision of neutrino oscillation measurements, only crude limits exist on the absolute magnitude of neutrino masses. Indeed, theoretical models span a large range of scenarios, from the degenerate case where the mass differences among flavors are small with respect to the absolute mass scale, to the hierarchical, where mass differences are of the same order as the mass themselves [7, 8, 9]. Although the neutrino mass scale is unknown, the present data on oscillations naturally leads to masses in the range $0.01<m_{\nu}<1 \mathrm{eV}$, as shown recently in Ref. [10] and others. In particular, the atmospheric neutrino oscillation results allow one to establish a model independent lower limit of $55 \mathrm{meV}$ for the mass of at least one of the neutrinos, thus defining the relevant mass scale. In general the measurement of the absolute value of neutrino masses should clearly be considered one of the most important frontiers of modern particle and astro physics.

Conceptually, the simplest way to search for the neutrino mass is based on the kinematic studies of the particles produced in weak decays such as tritium beta decay,

$$
{ }^{3} \mathrm{H} \rightarrow{ }^{3} \mathrm{He}+\mathrm{e}^{-}+\bar{\nu}_{e} .
$$

The best current limit on the $\nu_{e}$ mass using tritium is $m_{\nu_{e}} \leq 2.2 \mathrm{eV}$ as measured by the Mainz collaboration [11]. The design of a next generation spectrometer is being pursued by the KATRIN collaboration [12], with an expected sensitivity to the 
directly measured electron neutrino mass of $m_{\nu_{e}} \approx 0.35 \mathrm{eV}$.

Another conceptually simple, but not directly competitive, neutrino mass determination is based on the time of flight method using neutrinos from a nearby supernova. Large underground detectors like Super-Kamiokande, SNO and KamLAND [1, 2, 3] are particularly well equipped to perform such a measurement that, nonetheless, requires a supernova explosion in the right region of the universe. The most recent nearby supernova, SN1987a, produced enough neutrino detections to limit the neutrino mass to $\sim 23 \mathrm{eV}$. Such supernovae are expected to occur about once per century, limiting the technique's usefulness.

\subsection{Double beta decay}

Double beta decay is the transition between a nucleus of charge $\mathrm{Z}$ and mass number $\mathrm{A}$ (with both $\mathrm{A}$ and $\mathrm{Z}$ even) and one of same mass and charge $\mathrm{Z}+2$. Here we will limit ourselves to a review of results and problems, while a more complete treatment is given in several review articles $[13,14,15]$.

In its basic standard model form, double beta decay $(2 \nu \beta \beta)$ can be regarded as two $\beta^{-}$decays happening simultaneously, such that the process

$$
(Z, A) \rightarrow(Z+2, A)+e_{1}^{-}+e_{2}^{-}+\bar{\nu}_{e 1}+\bar{\nu}_{e 2}
$$

produces two leptons and two anti-leptons in the final state. While this second-order process is a standard manifestation of nuclear physics, its rate is expected to be proportional to $\left(G_{F} \cos \theta_{C}\right)^{4}$, where $G_{F}$ is the Fermi coupling constant and $\theta_{C}$ the Cabibbo angle. Hence, double beta decay would be an unobservably small correction to regular beta decay if it was not for a peculiarity in the nuclear mass function that energetically disallows the regular beta decay for some isotopes. As shown in Fig. 2.1 for mass $A=136$, nuclei with an odd number of protons and neutrons (odd-odd) and nuclei with an even numbers (even-even) are arranged on separate mass parabolae because of the different value of the pairing term in the nuclear binding energy. In this case, the direct $\beta$ transition $\left({ }^{136} \mathrm{Xe} \rightarrow{ }^{136} \mathrm{Cs}\right)$ is energetically forbidden, and the 


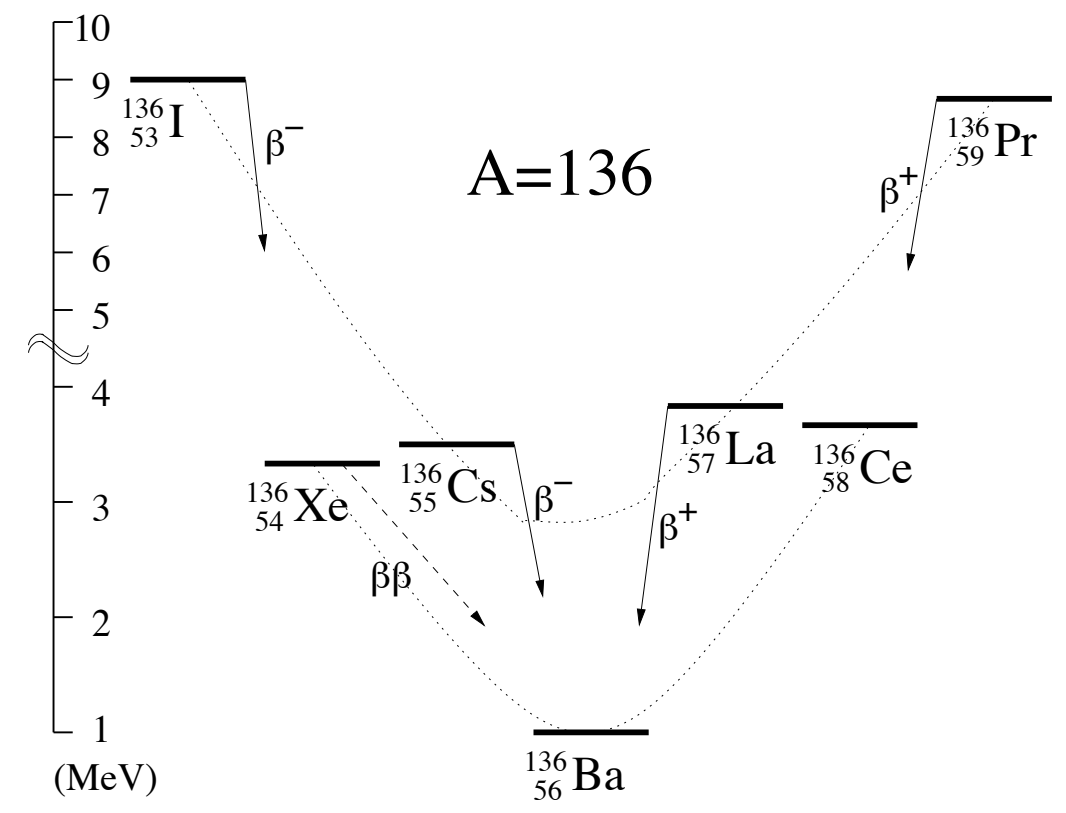

Figure 2.1: Simplified atomic mass scheme for nuclei with $A=136$. The parabolae connecting the odd-odd and even-even nuclei are shown. While ${ }^{136} \mathrm{Xe}$ is stable to ordinary beta decay, it can decay into ${ }^{136} \mathrm{Ba}$ by double-beta decay.

direct double beta decay process can be observed. Although this situation is common, only for 11 nuclei is the total available lepton energy $(Q)$ greater than $2 \mathrm{MeV}$ so that the decay can be observed in favorable conditions. $2 \nu \beta \beta$ decay has been observed in 11 different isotopes and the half life measured can be used to verify nuclear structure models in the presence of second order weak processes. Measurements agree within the range of theoretical predictions.

If lepton number is not conserved and neutrinos have finite mass, then a different kind of double beta decay can occur with no neutrinos in the final state $(0 \nu \beta \beta)$ as illustrated in Fig. 2.2. This process requires the neutrinos to be Majorana particles, since the anti-neutrino emitted at $A$ must be identical to the neutrino absorbed at $B$. Furthermore, since Standard Model weak interactions only couple to left-handed states, the neutrino must have a finite mass so that it is not a helicity eigenstate.

The Majorana nature of the neutrino has been the subject of many articles in the literature. Various reasonable extensions of the Standard Model predict the existence 

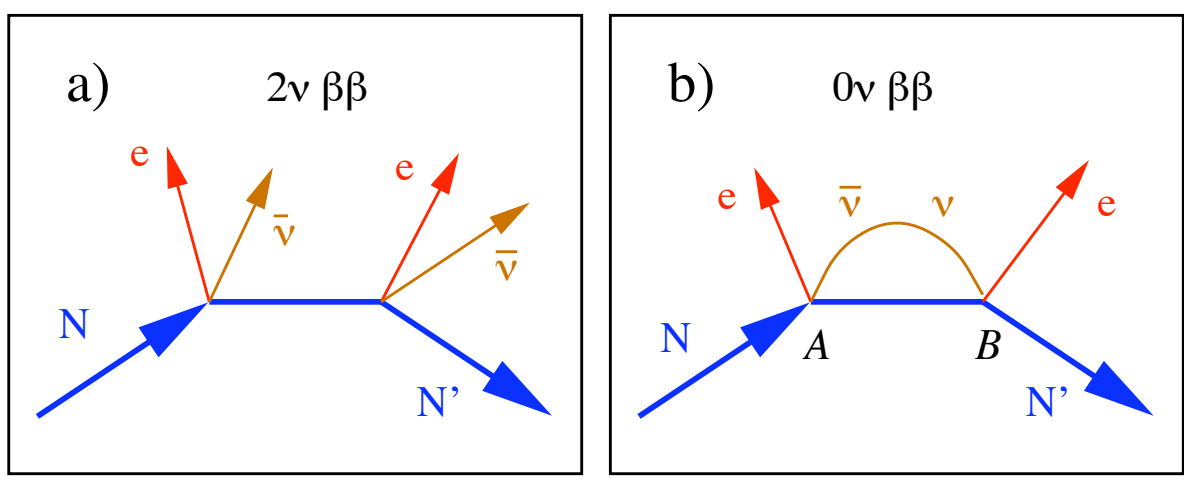

Figure 2.2: Feynman diagrams for a) two neutrino and (b) zero neutrino double beta decay.

of neutrino mass and most such extensions lead to the expectation that massive neutrinos are Majorana particles. Consequently, the phenomenon of mixing, analogous to the Cabibbo-Kobayashi-Maskawa mixing among quarks, is expected to govern their weak interactions. Ref. [16] presents a more complete review of the current literature. For our purposes, it suffices to say that a massive neutrino must have a Majorana mass component unless an unknown symmetry prohibits lepton number violation. Many extensions to the Standard Model, in particular the "See-Saw mechanism" (c.f. $\S 1.4$ of Ref. [17]), require neutrinos to be Majorana particles in order to explain their relative lightness.

Assuming that neutrinos are in fact Majorana particles, the neutrino oscillation data limits the possible Majorana neutrino mass depending on the assumed mass spectrum. The neutrino oscillation data provides two mass differences: the solar neutrino mass splitting $\Delta m_{12}^{2}=7.1 \times 10^{-5} \mathrm{eV}^{2}$, and the atmospheric neutrino mass splitting $\Delta m_{23}^{2}=2.0 \times 10^{-3} \mathrm{eV}^{2}$. These splittings limit the possible distributions of the neutrino masses $m_{1}, m_{2}$, and $m_{3}$ to the "Normal", "Inverted" and "Degenerate" scenarios. In the Normal scenario, the two lightest neutrinos have masses on the order of $\sqrt{\Delta m_{12}^{2}}$ and are much lighter than the third neutrino; ie. $m_{1} \sim m_{2} \ll m_{3}$. In the Inverted scenario, there is one light neutrino seperated from two heavy neutrinos by $\sqrt{\Delta m_{12}^{2}}, m_{1} \ll m_{2} \sim m_{3}$. Finally, in the Degenerate scenario all three neutrinos have the same mass, $m_{1} \sim m_{2} \sim m_{3} \gg \sqrt{\Delta m_{12}^{2}}$, and the neutrino mass value is 
limited only by the tritium beta decay measurements. The effect of these hierarchies on the mass sensitivity of double beta decay is shown in Fig. 2.3. Note that the

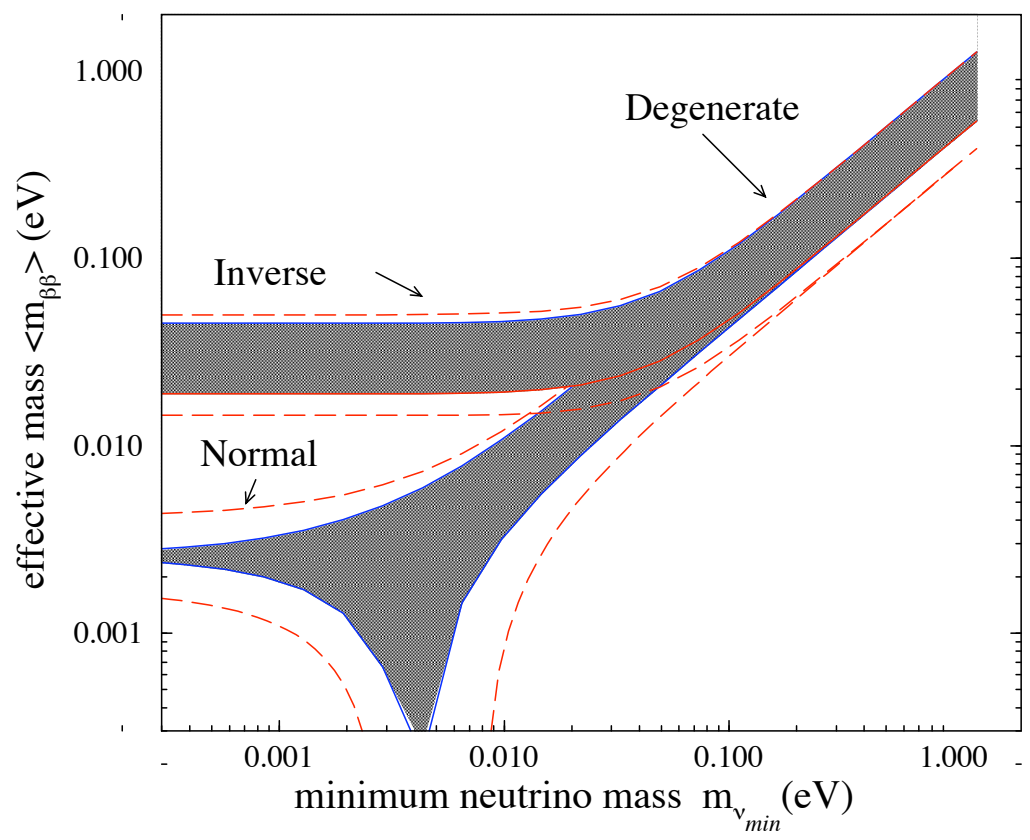

Figure 2.3: Dependence of the effective Majorana neutrino mass $\left\langle m_{\beta \beta}\right\rangle$ on the absolute mass of the lightest neutrino adapted from Ref. [18]. The solid region indicates the range of $0 \nu \beta \beta$ mass related to the unknown Majorana phases, while the dashed lines include the $1 \sigma$ errors on the oscillation parameters.

$0 \nu \beta \beta$ sensitivity to the neutrino mass depends strongly on the Majorana phases for the smallest masses. Cancellations in the Majorana phases can reduce $\left\langle m_{\beta \beta}\right\rangle$ to zero at low masses. For the heavier masses observed in the degenerate case, the lack of knowledge of the Majorana phases is reflected in the range of neutrino masses which give rise to a given $\left\langle m_{\beta \beta}\right\rangle$.

If the lepton number non-conservation is due to spontaneous symmetry breaking, double beta decay could occur as

$$
(Z, A) \rightarrow(Z+2, A)+e_{1}^{-}+e_{2}^{-}+\chi
$$

where the particle $\chi$ (Majoron) is the Goldstone boson of the lepton number symmetry 


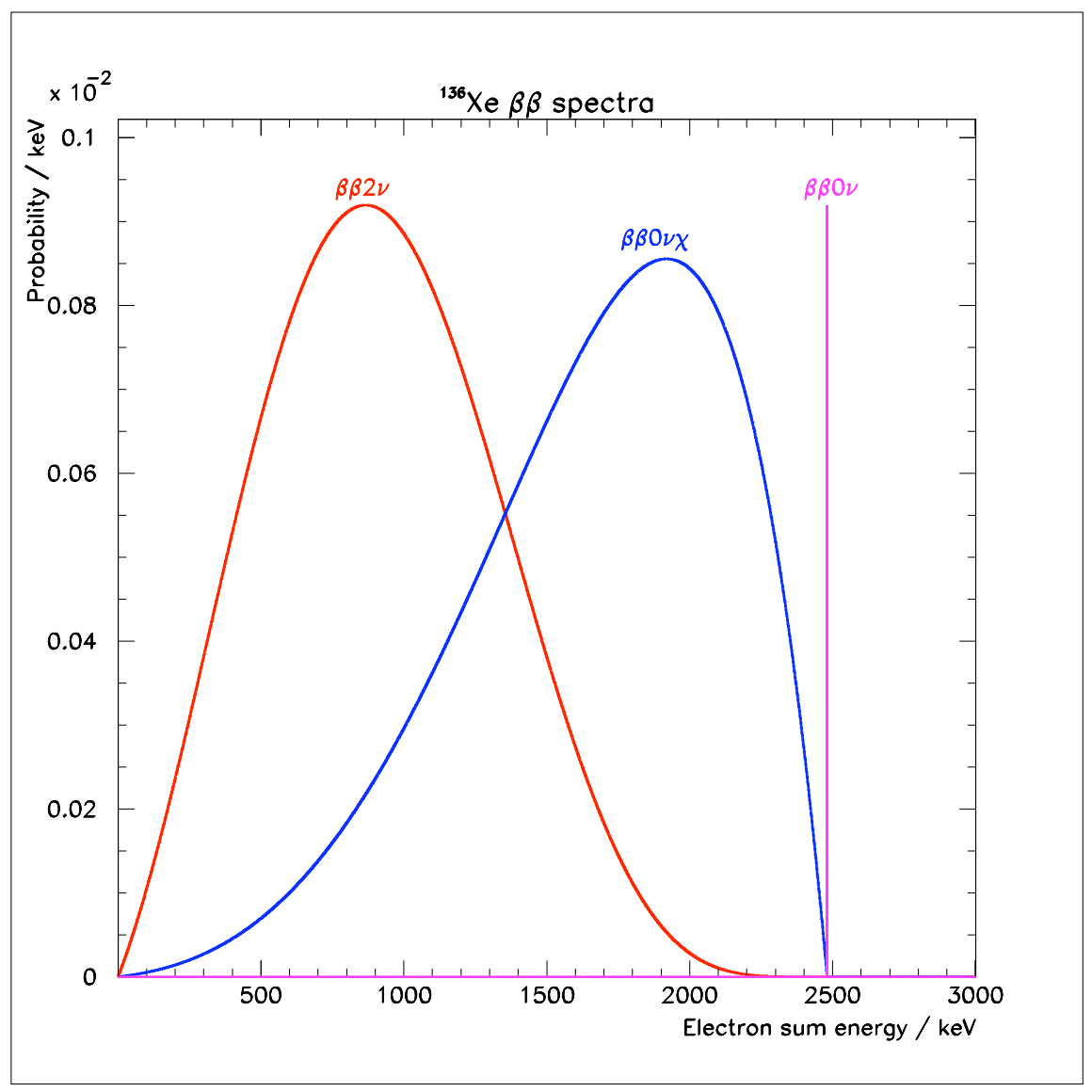

Figure 2.4: The two neutrino, zero neutrino, and Majoran double beta decay modes. The only method to distinguish the modes is via kinematic measurement.

breaking. In all models, $\chi$ is a weakly interacting particle that cannot be detected. Similarly, new, exotic particles can be replaced for the neutrino in Fig. 2.2(b) and produce $0 \nu \beta \beta[19,20]$. Regardless of the mechanism, it is in general true that the observation of $0 \nu \beta \beta$ always implies the discovery of previously unknown physics. Indeed, the connection between neutrino masses or, in general, new physics beyond the "standard model" and the existence of $0 \nu \beta \beta$ drives the interest in this process, and by extension, for EXO. Note that the only way to distinguish the various decay processes is through the electron energy spectrum, as shown in Fig. 2.4.

If the $0 \nu \beta \beta$ decay process occurs, then the effective Majorana neutrino mass $\left\langle m_{\nu}\right\rangle$ 
is related to the decay half-life $T_{1 / 2}^{0 \nu \beta \beta}$ as

$$
\left\langle m_{\nu}\right\rangle^{2}=\left(T_{1 / 2}^{0 \nu \beta \beta} G^{0 \nu \beta \beta}\left(E_{0}, Z\right)\left|M_{G T}^{0 \nu \beta \beta}-\frac{g_{V}^{2}}{g_{A}^{2}} M_{f}^{0 \nu \beta \beta}\right|^{2}\right)^{-1}
$$

where $G^{0 \nu \beta \beta}\left(E_{0}, Z\right)$ is a known phase space factor depending on the end-point energy $E_{0}$ and the nuclear charge $Z . M_{G T}^{0 \nu \beta \beta}$ and $M_{F}^{0 \nu \beta \beta}$ are the Gamow-Teller and Fermi nuclear matrix elements for the process which must be numerically calculated. We have defined

$$
\left\langle m_{\nu}\right\rangle=\sum_{i} m_{i} U_{e i}^{2}
$$

with the complex lepton sector mixing matrix, $U$, and the masses of the individual Majorana neutrinos, $m_{i}$. Consequently, $0 \nu \beta \beta$ decay is sensitive to the masses of all the neutrino flavors, providing there are no phase cancellations among the Majarona mixing angles.

Several characteristics of Eqs. $2.4 \& 2.5$ merit particular attention. Since the mixing matrix enters Eq. 2.5 as a simple square and not as an absolute square, the so-called Majorana phases can cause cancellations in the incoherent summation. The phases are responsible for the range in sensitivities in Fig. 2.3. From an experimental perspective, Eq. 2.4 demonstrates that the sensitivity to neutrino mass improves only with the square root of the half-life sensitivity.

Difficulties in the nuclear models used to calculate the matrix elements gives some uncertainty to the value of $\left\langle m_{\nu}\right\rangle$ derived for a particular $T_{1 / 2}^{0 \nu \beta \beta}$. In order to evaluate the matrix elements, the wave function of the nucleus before and after the double beta decay must be calculated, as well as an operator connecting the two states. For the lightest of the decay candidates, ${ }^{48} \mathrm{Ca}$, the configuration of 48 nucleons must be calculated, a technically challenging task. Two models are used to calculate matrix elements: the Nuclear Shell Model (NSM) [21] and the Quasi-Random Phase Approximation (QRPA) [22]. Historically, the matrix elements calculations differ by $\sim 10$, in large part because knowledge of the $2 \nu \beta \beta$ matrix elements does not directly contribute to calculations of the $0 \nu \beta \beta$ matrix elements. However, Rodin et al. [22] have recently developed a technique to reduce the major source of uncertainty in the 


\begin{tabular}{|l|c|c|c|c|}
\hline \hline$\beta \beta$ decay candidate & $\mathrm{Q}(\mathrm{MeV})$ & Isot. Abund. $(\%)$ & $1 / G^{2 \nu \beta \beta}(\mathrm{yr})$ & $1 / G^{0 \nu \beta \beta}(\mathrm{yr})$ \\
\hline${ }^{48} \mathrm{Ca} \rightarrow{ }^{48} \mathrm{Ti}$ & 4.271 & 0.187 & $2.52 \times 10^{16}$ & $4.10 \times 10^{24}$ \\
${ }^{76} \mathrm{Ge} \rightarrow{ }^{76} \mathrm{Se}$ & 2.040 & 7.8 & $7.66 \times 10^{18}$ & $4.09 \times 10^{25}$ \\
${ }^{82} \mathrm{Se} \rightarrow{ }^{82} \mathrm{Kr}$ & 2.995 & 9.2 & $2.30 \times 10^{17}$ & $9.27 \times 10^{24}$ \\
${ }^{96} \mathrm{Zr} \rightarrow{ }^{96} \mathrm{Mo}$ & 3.350 & 2.8 & $5.19 \times 10^{16}$ & $4.46 \times 10^{24}$ \\
${ }^{100} \mathrm{Mo} \rightarrow{ }^{100} \mathrm{Ru}$ & 3.034 & 9.6 & $1.06 \times 10^{17}$ & $5.70 \times 10^{24}$ \\
${ }^{110} \mathrm{Pd} \rightarrow{ }^{110} \mathrm{Cd}$ & 2.013 & 11.8 & $2.51 \times 10^{18}$ & $1.86 \times 10^{25}$ \\
${ }^{116} \mathrm{Cd} \rightarrow{ }^{116} \mathrm{Sn}$ & 2.802 & 7.5 & $1.25 \times 10^{17}$ & $5.28 \times 10^{24}$ \\
${ }^{124} \mathrm{Sn} \rightarrow{ }^{124} \mathrm{Te}$ & 2.228 & 5.64 & $5.93 \times 10^{17}$ & $9.48 \times 10^{24}$ \\
${ }^{130} \mathrm{Te} \rightarrow{ }^{130} \mathrm{Xe}$ & 2.533 & 34.5 & $2.08 \times 10^{17}$ & $5.89 \times 10^{24}$ \\
${ }^{136} \mathrm{Xe} \rightarrow{ }^{136} \mathrm{Ba}$ & 2.479 & 8.9 & $2.07 \times 10^{17}$ & $5.52 \times 10^{24}$ \\
${ }^{150} \mathrm{Nd} \rightarrow{ }^{150} \mathrm{Sm}$ & 3.367 & 5.6 & $8.41 \times 10^{15}$ & $1.25 \times 10^{24}$ \\
\hline \hline
\end{tabular}

Table 2.1: Double beta decay phase space factors for isotopes with $Q>2 \mathrm{MeV}$. For ${ }^{48} \mathrm{Ca}$ and ${ }^{96} \mathrm{Zr}, \beta$ decay is kinematically allowed but spin suppressed.

calculation of the $0 \nu \beta \beta$ mode by comparing QRPA calculations to measured $2 \nu \beta \beta$ decay rates.

It is important to realize that a positive measurement of $0 \nu \beta \beta$ or $\chi \beta \beta$ has substantial impact on all of particle physics and hence requires a high degree of confidence in the data. A meaningful experiment must use a technique capable of producing solid results with negligible effects due to background.

\subsection{Present status of $0 \nu \beta \beta$ searches}

Several past experiments have searched for $0 \nu \beta \beta$ decay. To date, no generally accepted positive result has been reported. The phase-space factor $G^{0 \nu \beta \beta}$ is a steep function of the end-point energy $\left(G^{0 \nu \beta \beta} \simeq\left(E_{0}^{5} / 30-2 E_{0}^{2} / 3+E_{0}-2 / 5\right)\right.$ in the PrimakoffRosen approximation [17]), and in practice only isotopes with the largest $Q$ values can be used. Also constraining experiments are the facts that $0 \nu \beta \beta$ candidates must have reasonable natural isotopic abundance and the experiments are generally performed in relatively deep underground sites to reduce cosmic-ray backgrounds. In Table 2.1, we list the decay candidates with $Q>2 \mathrm{MeV}$ along with their isotopic abundance and phase space factors for $0 \nu \beta \beta$ and $2 \nu \beta \beta$ decays. 


\begin{tabular}{|l|c|c|}
\hline \hline$\beta \beta$ decay candidate & $T_{1 / 2}^{0 \nu \beta \beta}(\mathrm{yr})$ & Reference \\
\hline${ }^{48} \mathrm{Ca}$ & $>9.5 \times 10^{21}(76 \% \mathrm{CL})$ & {$[23]$} \\
${ }^{76} \mathrm{Ge}^{*}$ & $>1.9 \times 10^{25}(90 \% \mathrm{CL})$ & {$[4]$} \\
${ }^{82} \mathrm{Se}$ & $>1.9 \times 10^{23}(90 \% \mathrm{CL})$ & {$[24]$} \\
${ }^{100} \mathrm{Mo}$ & $>3.5 \times 10^{23}(90 \% \mathrm{CL})$ & {$[24]$} \\
${ }^{116} \mathrm{Cd}$ & $>1.7 \times 10^{23}(90 \% \mathrm{CL})$ & {$[25]$} \\
${ }^{130} \mathrm{Te}^{*}$ & $>1.8 \times 10^{24}(90 \% \mathrm{CL})$ & {$[26]$} \\
${ }^{136} \mathrm{Xe}^{*}$ & $>1.2 \times 10^{21}(90 \% \mathrm{CL})$ & {$[27]$} \\
${ }^{150} \mathrm{Nd}$ & $>1.2 \times 10^{21}(90 \% \mathrm{CL})$ & {$[28]$} \\
\hline \hline
\end{tabular}

Table 2.2: Recent half-life results for the most sensitive $0 \nu \beta \beta$ experiments. Isotopes marked with ${ }^{*}$ use the source as the electron calorimeter and are discussed further in the text.

In Table 2.2, we show the best sensitivities reached to date for $0 \nu \beta \beta$ decay. Although several experiments have been performed in which the $0 \nu \beta \beta$ source is external to the detecting apparatus, there are clear advantages in schemes where the source also serves as the electron detector. Indeed the experiments showing the best sensitivity all utilize this principle.

$0 \nu \beta \beta$ decay in ${ }^{76} \mathrm{Ge}$ has been studied using very large volume germanium diode ionization counters. In this case the detector and the source are made of the same material and the event tagging is purely calorimetric. The germanium detectors have been built out of isotopically enriched ${ }^{76} \mathrm{Ge}$, improving the sensitivity by increasing the ratio of source mass to detector mass. The isotopic separation, the clean conditions required for large, diode-quality crystals, and the high energy help provide low intrinsic detector background. However, germanium offers no proven technique to reduce the remaining backgrounds to a level adequate for $0 \nu \beta \beta$ searches. The HeidelbergMoscow experiment at Gran Sasso [32] has achieved the highest $m_{\nu}$ sensitivity with detectors totaling $11 \mathrm{~kg}\left(86 \%{ }^{76} \mathrm{Ge}\right)$ taking data for $14 \mathrm{yrs}$. Total exposure for the most recently reported results is $71.7 \mathrm{~kg}$ y. Typical energy resolutions of $\mathrm{FWHM} \simeq 3$ $\mathrm{keV}$ at $2 \mathrm{MeV}$ are achieved, the best figure obtained in any detector. The excellent energy resolution is particularly useful in discriminating between the $0 \nu \beta \beta$ (monoenergetic) and $2 \nu \beta \beta$ (energy continuum) spectra. A previous paper, Ref. [4] reports 
the total background observed in the detector as $0.3 \mathrm{~kg}^{-1} \mathrm{yr}^{-1} \mathrm{FWHM}^{-1}$. The halflife limit of $5.7 \times 10^{25}$ yrs gives a neutrino mass limit of $\left\langle m_{\nu}\right\rangle<0.2(0.6) \mathrm{eV}$ using the "Quasi-Particle Random Phase Approximation" (QRPA) [29, 30] ("Nuclear Shell Model" or NSM [31]) for the nuclear matrix element calculations.

A subset of the Heidelberg-Moscow collaboration has reported a positive $0 \nu \beta \beta$ signal at the $2039 \mathrm{keV}$ endpoint of the ${ }^{76} \mathrm{Ge}$ decay and claimed to have $4.2 \sigma$ significance [32]. Unfortunately, the data requires substantial background simulation and removal and does not satisfy the criterion of "solid results with negligible background" required for a discovery of the magnitude of $0 \nu \beta \beta$. Many members of the neutrino physics community, including members of the original Heidelberg-Moscow collaboration, have published a note [18] refuting aspects of the methodology employed in the original $0 \nu \beta \beta$ claim published in 2001 [4]. Although the new analysis published in 2004 with improved statistics addresses some of these issues [32], the result is still quite controversial.

${ }^{130} \mathrm{Te}$ has the advantage of a rather large isotopic fraction in natural tellurium $(34.5 \%)$. However the realization of the particle detector is more exotic than for the other nuclei. A tellurium electron calorimeter detects the temperature rise from deposited electron energy in a $\mathrm{TeO}_{2}$ crystal. Since heat capacity is proportional to $T^{3}$, this is only possible at very low temperature; in cryogenic conditions even the very small energy deposited by the electrons causes a detectable temperature increase. In principle the ultimate energy resolution of such detectors is superior to germanium diodes, although this has not yet been practically demonstrated in large crystals. The present limit [26] (Table 2) was obtained with 62 crystals of tellurium oxide, totaling $40.7 \mathrm{~kg}$, running at a temperature of $8 \mathrm{mK}$. The detectors have a resolution FWHM $\sim 8 \mathrm{keV}$ at $2.5 \mathrm{MeV}$ and logged $10.85 \mathrm{~kg}$ y of ${ }^{130} \mathrm{Te}$ in the Gran Sasso Laboratory [33, 26]. The background reported is $1.44 \pm 0.08 \mathrm{~kg}^{-1} \mathrm{yr}^{-1} \mathrm{FWHM}^{-1}$. The resulting half-life limit of $T_{1 / 2}>1.8 \times 10^{24}$ yrs corresponds to a neutrino mass limit of $\left\langle m_{\nu}\right\rangle<0.2(1.1) \mathrm{eV}$ in the QRPA (NSM) approximation.

The best sensitivity for ${ }^{136} \mathrm{Xe}$ was achieved in a $5 \mathrm{~atm}$ TPC containing $5.3 \mathrm{~kg}$ of Xe enriched to $63 \%{ }^{136} \mathrm{Xe}$, operated inside the Gotthard road tunnel in Switzerland [34]. A sketch of this detector is shown in Fig. 2.5. The total running period was $1.5 \mathrm{yr}$ and 


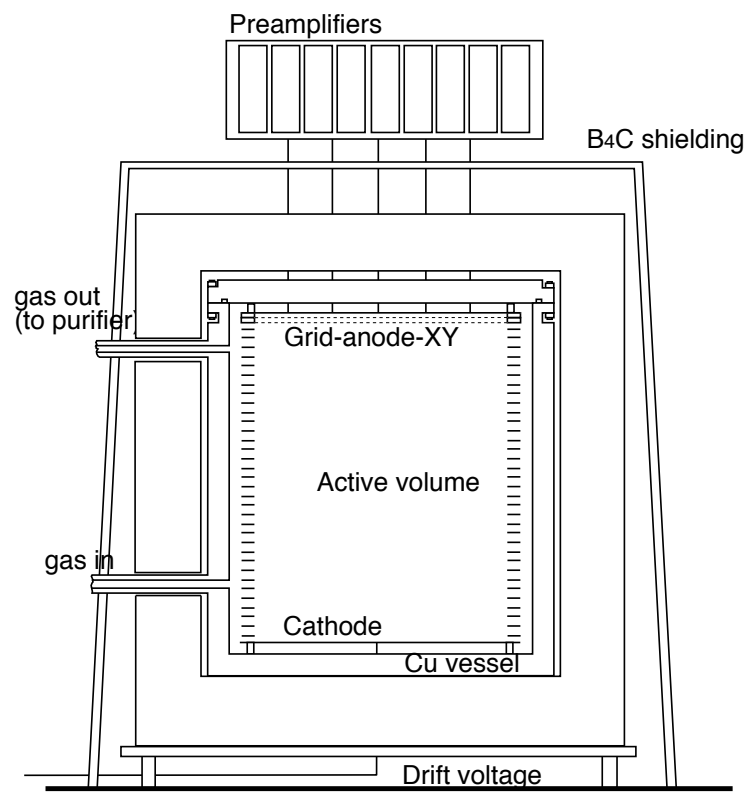

Figure 2.5: Schematic of the Gotthard TPC discussed in the text. The TPC used 5.3 $\mathrm{kG}$ of ${ }^{136} \mathrm{Xe}$ at $5 \mathrm{~atm}$.

the energy resolution was FWHM $=6.6 \%$ at $1.592 \mathrm{MeV}(105 \mathrm{keV})$. The background was $2.5 \mathrm{~kg}^{-1} \mathrm{yr}^{-1} \mathrm{FWHM}^{-1}$. The superior tracking capability of this technique was vital to reduce backgrounds. The resulting half-life limit of $T_{1 / 2}>4.4 \times 10^{23} \mathrm{yr}$ corresponds to a neutrino mass limit of $\left\langle m_{\nu}\right\rangle<2.2(5.2) \mathrm{eV}$ in the QRPA (NSM) approximation.

\subsection{EXO detector design concepts}

The most sensitive searches for $0 \nu \beta \beta$ to date have used $\sim 10 \mathrm{~kg}$ of nearly-pure isotopic species; hence further substantial progress can only be achieved through an ambitious program where tons of an appropriate isotope are monitored for a relatively long time. Indeed, it is legitimate to ask whether backgrounds can be reduced to a small enough level such that full use can be made of the large mass of active material. This point is essential: in a background-free experiment the sensitivity to the half-life $T_{1 / 2}^{0 \nu \beta \beta}$ is directly proportional to the number of nuclei, $N$ and exposure time t, so that the 
neutrino mass sensitivity scales as:

$$
\left\langle m_{\nu}\right\rangle \propto 1 / \sqrt{T_{1 / 2}^{0 \nu \beta \beta}} \propto 1 / \sqrt{N t}
$$

However, unless a qualitatively new technique is used, the small background observed in current experiments will increase with the mass and exposure time to become the limiting factor. If we crudely assume that backgrounds increase linearly with $N t$, our measurement sensitivity to $T_{1 / 2}^{0 \nu \beta \beta}$ becomes limited by the statistical fluctuations in the background, $T_{1 / 2}^{0 \nu \beta \beta} \propto \sqrt{N t} / N t$. For this case, the neutrino mass sensitivity scales as

$$
\left\langle m_{\nu}\right\rangle \propto 1 / \sqrt{T_{1 / 2}^{0 \nu \beta \beta}} \propto 1 /(N t)^{1 / 4}
$$

Note that this analysis represents an extreme case since there will be an improvement in some backgrounds as the detector self-shielding increases. A more realistic sensitivity lies between the optimistic -1/2 exponent of Eq. 2.6 and the pessimistic $-1 / 4$ exponent of Eq. 2.7. Nonetheless, in order to achieve the maximum sensitivity for a given source mass, qualitatively new methods to reduce the backgrounds must be found.

Within this context, ${ }^{136} \mathrm{Xe}$ is an ideal isotope for a multi-ton double beta decay experiment since:

- it is one of the easiest isotopes to enrich;

- it represents a good ionization detecting medium with high energy resolution;

- it exhibits substantial scintillation that can be used to tag the event time;

- it has no long lived isotopes to activate;

- it can be easily and continuously purified with a variety of different techniques;

- and, most importantly, it produces a final state, ${ }^{136} \mathrm{Ba}$, that can be identified using optical spectroscopy.

The additional technique of Ba tagging will improve the already low specific background of Xe TPCs, minimizing the $0 \nu \beta \beta$ decay background and maximizing the 
$\left\langle m_{\nu}\right\rangle$ sensitivity.

The isotopic enrichment of ${ }^{136} \mathrm{Xe}$ deserves extra consideration, since the enriched source material will be the single most expensive component of all next generation $0 \nu \beta \beta$ experiments. ${ }^{76} \mathrm{Ge}$ and ${ }^{136} \mathrm{Xe}$ both have $<10 \%$ natural abundance and will require substantial enrichment for use in an experiment. With $34 \%$ natural abundance, ${ }^{130} \mathrm{Te}$ can be used in its natural state. Xenon enrichment is particularly simple and benefits from two serendipitous properties: xenon is an inert gas at the operating temperature and pressure of ultra-centrifuges, and ${ }^{136} \mathrm{Xe}$ is the heaviest isotope of xenon, allowing the enrichment to be done in a single pass through a cascade of centrifuges. In contrast, germanium enrichment requires extensive chemistry to create a gas for use in the centrifuges, reducing the enrichment efficiency and adding cost. The EXO collaboration has already enriched a $200 \mathrm{~kg}$ sample of xenon to $80 \%{ }^{136} \mathrm{Xe}$ to be used in a prototype detector.

\subsection{Liquid TPC concept}

The high density of liquid xenon makes makes a liquid phase detector particularly attractive for an underground experiment. At the modest cryogenic temperature of $160 \mathrm{~K}$, the density of liquid xenon is $3 \mathrm{~g} / \mathrm{cm}^{3}$; an entire 10 ton detector requires a volume of only $\sim 3 \mathrm{~m}^{3}$. Consequently, our research program is focused on developing the technologies required to implement such a detector. The major disadvantage of a liquid TPC is the difficulty of performing barium spectroscopy in liquid, in part because of uncertainty about the barium ionization state in the liquid and in part because the barium resonance lines broaden, reducing the signal and requiring substantially larger lasers than gas or vacuum spectroscopy. To avoid these issues, we propose extracting the single barium ions from the liquid to an external spectroscopic analysis cell.

A conceptual scheme for realizing a full scale ( $>1$ ton) liquid xenon TPC is shown in Fig. 2.6. This particular concept consists of a toroidal cryostat for the xenon, although a rectangular version would also be possible. The inner wall at large radius functions as a cathode, and the inner wall at small radius is the anode, 
segmented in both azimuth $(\phi)$ and in height (the z direction). In a high voltage field, ionization produced by $\beta$ electrons drifts to the anodes where the charge is collected and measured to determine the event energy. The event's position information is provided by the anode segmentation (for reconstruction of $\phi$ and $z$ ), and by the measured drift time (for reconstruction of the radial position $\mathrm{r}$ ). The bottom of the vessel is covered with closely spaced, Large Area Avalanche Photo Diodes (LAAPDs) to collect scintillation light. The signals from the LAAPDs provides a timing signal for the electron drift time measurement as well as additional calorimetric information to supplement the ionization signal and improve the calorimetric resolution [36].

In this concept, a robotic arm equipped with an electrostatic ion-extraction probe extracts the barium ion from the location of a candidate ionization event. The probe delivers the ion to an ion trap, located as close as possible to the liquid xenon, for spectroscopic identification. The integration of ion trapping and spectroscopy with the liquid xenon TPC is one of the greatest development challenges facing the EXO collaboration, and the main topic of this thesis. Specifically, we seek to answer the age-old question, "what is the maximum xenon pressure compatible with single ion trapping and detection?"

\subsection{Expected detector performance}

The experiment will be located in an underground site in order to provide shielding against cosmic radiation. For the purposes of these estimates, we consider as a minimum over burden the 2000 meter water equivalent (mwe) shielding of DOE's Waste Isolation Pilot Plant (WIPP). The overburden reduces the the cosmic ray muon flux to $4.09 \pm .4 \times 10^{-3} \mathrm{~m}^{-2} \mathrm{~s}^{-1}[35]$, corresponding to $\leq 0.01 \mathrm{~Hz}$ through the detector. Muons have a very distinctive signature and should be rejected based on a number of features; they will not generate a substantial signal in the detector.

Some background will be caused by by muon spallation in the structures (rock and other materials) outside the detector. These fast neutrons can enter the detector produce spallation reactions on the xenon, carbon and hydrogen nuclei contained in the TPC. While the original muon and the neutron trail may go undetected the 


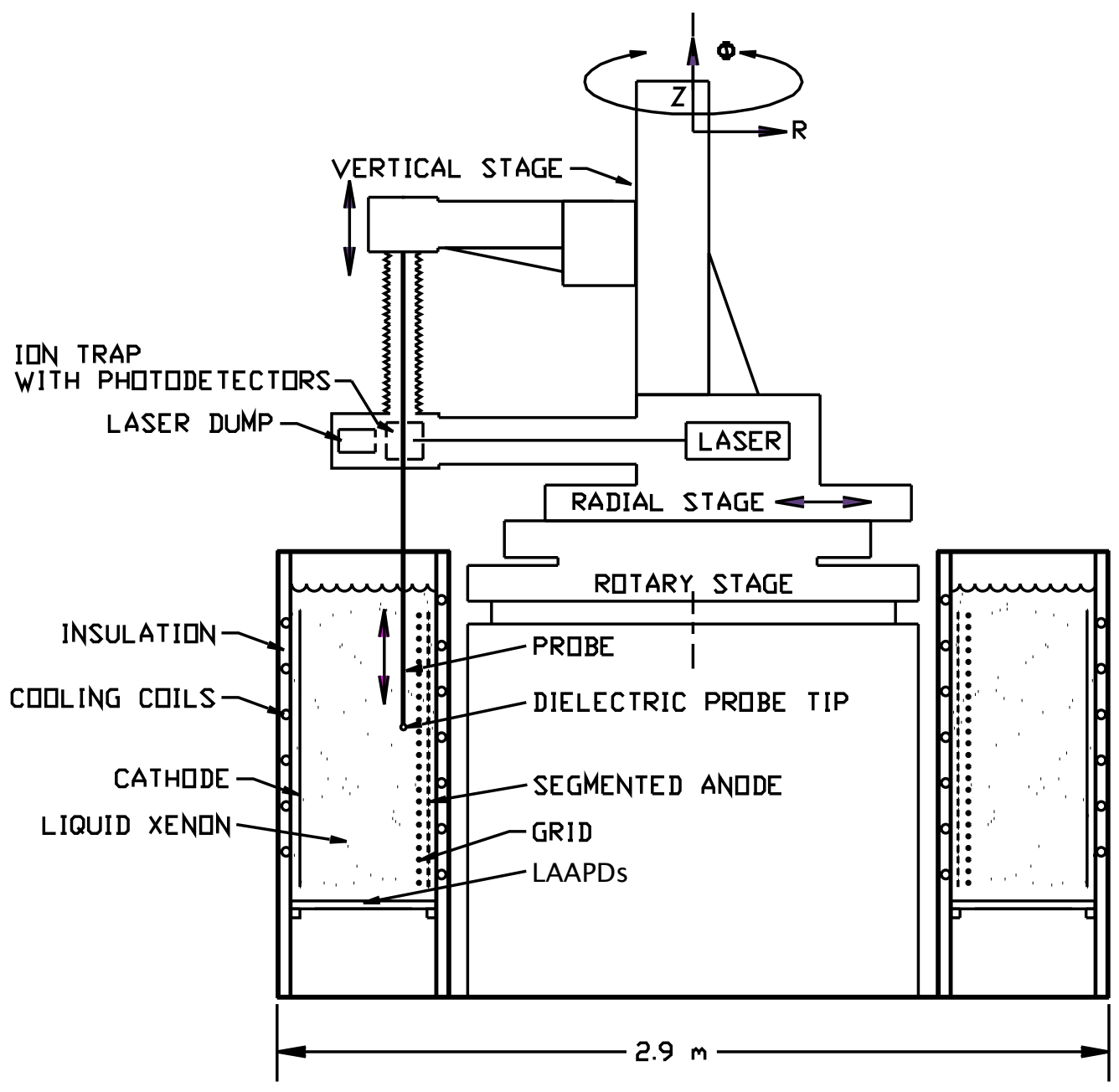

Figure 2.6: Conceptual design of a liquid xenon detector with barium extraction. 
spallation processes provide very distinct short, high-ionization tracks that are easy to distinguish and reject using a TPC's tracking capabilities.

More serious is the background from natural radioactivity, either produced outside the TPC (mainly by the rock or concrete), or inside (mainly ${ }^{222} \mathrm{Rn}$ and, possibly, ${ }^{85} \mathrm{Kr}$ and $\left.{ }^{42} \mathrm{Ar}\right)$. Preliminary estimates indicate that external shielding alone should be sufficient for the experiment, for instance a $25 \mathrm{~cm}$ thick lead enclosure. Substantial additional shielding is provided by a layer of cryogenic refrigerant (such as HFE7000, manufactured by the 3M company) between the shielding and detector which combines excellent thermal properties with high density and low radioactivity.

For the following estimates we conservatively assume that the TPC itself (without Ba-tagging system) will have the same specific rate of mis-identified background as the Gotthard experiment, and we rely on barium tagging to keep the backgrounds from radioactivity to a negligible level. This hypothesis is conservative since the larger volume of our TPC provides better self-shielding for the externally produced radiation than observed at Gotthard. Internal backgrounds will be limited by careful choice of construction materials (essentially only copper, acrylic, Teflon and polyamide films) and by removing contaminants from the xenon. We assume the energy resolution to be no worse than the $\mathrm{E}=1.6 \%$ obtained by Conti et al. [36] by correlating the ionization and scintillation spectrum. Since the full scale detector will have substantially improved scintillation light collection this assumption underestimates the probable resolution. Consequently, we also give an "asymptotic sensitivity" that will be obtained with a more aggressive resolution of $1 \%$ and a very long data-taking with a 10 ton detector (100 ton yr exposure). From the size and geometry of the chamber we obtain an efficiency for fully contained events of $70 \%$. We note that there will be no residual $\mathrm{Ba}+$ in the detector since barium is a reactive metal with a negligible vapor pressure at room temperature. Any barium produced will drift in the electric field to the cathode walls where it will form a chemical bond and disappear.

It is interesting to note that for very large quantities of material the $2 \nu \beta \beta$ mode dominates the $0 \nu \beta \beta$ decay background. As already remarked this "background" is obviously not suppressed by the laser tagging methods, and, since its only distinctive feature is the electron energy spectrum (see Fig. 2.4), energy resolution is the only 


\begin{tabular}{|l|l|l|l|l|l|l|l|}
\hline $\begin{array}{l}\text { Isotope and } \\
\text { Reference }\end{array}$ & $\begin{array}{l}\text { Total } \\
\text { Mass } \\
(\mathrm{kG})\end{array}$ & $\begin{array}{l}\text { Enrich. } \\
\text { grade } \\
(\%)\end{array}$ & $\begin{array}{l}\text { Det. } \\
\text { Eff. } \\
(\%)\end{array}$ & $\begin{array}{l}\text { Meas. } \\
\text { time } \\
(\mathrm{yr})\end{array}$ & $\begin{array}{l}\text { Bkgnd } \\
(\text { cts. })\end{array}$ & $T_{1 / 2}^{0 \nu \beta \beta}(\mathrm{yr})$ & $\begin{array}{l}\left\langle m_{\nu}\right\rangle \\
\text { QRPA(NSM) } \\
\mathrm{eV}\end{array}$ \\
\hline${ }^{76} \mathrm{Ge}[4]$ & 11 & 86 & 75 & 2.6 & 0.3 & $5.7 \times 10^{25}$ & $0.2(0.6)$ \\
${ }^{130} \mathrm{Te}[26]$ & 40.7 & 34.5 & & 0.26 & 15.6 & $4.4 \times 10^{23}$ & $0.2(1.1)$ \\
${ }^{136} \mathrm{Xe}[34]$ & 5.3 & 63 & & 1.5 & 1.3 & $4.4 \times 10^{23}$ & $2.2(5.2)$ \\
\hline $\begin{array}{l}{ }^{136} \mathrm{Xe} \\
\sigma_{E} / E=1.6 \% \\
{ }^{136} \mathrm{Xe} \\
\sigma_{E} / E=1.0 \%\end{array}$ & 1000 & 80 & 70 & 5 & 0.5 & $2.0 \times 10^{27}$ & $0.050(0.068)$ \\
\hline
\end{tabular}

Table 2.3: Predicted sensitivity of the EXO detector to the $0 \nu \beta \beta$ half-life, compared to the current best sensitivities.

effective tool to suppress this background.

While the $2 \nu \beta \beta$ mode is interesting in its own right, a reasonable resolution on the total energy measurement removes it from the $0 \nu \beta \beta$ mode sample and allows a background-free measurement. We select events in the interval $I_{+}=\left[Q_{\beta \beta}, Q_{\beta \beta}+\right.$ $\left.2 \sigma_{E}\right]$ where $\sigma_{E}=1.6 \%$ in the first phase and $\sigma_{E}=1.0 \%$ in the latter phase. The asymmetric interval is chosen to maximize the $0 \nu \beta \beta$ mode relative to $2 \nu \beta \beta$, which has a steeply decreasing spectrum in this region. We then compute the number of $2 \nu \beta \beta$ decay events left within the $0 \nu \beta \beta$ sample for each case. For the first phase we have 0.5 remaining $2 \nu \beta \beta$ events, while $0.72 \nu \beta \beta$ events remain in the $100 \mathrm{yr}$ ton data sample. These backgrounds are statistically subtracted using the $2 \nu \beta \beta$ rate prediction from other regions of the energy spectrum. Although this procedure relies on a good understanding of the resolution function, we conservatively estimate our total number of $0 \nu \beta \beta$ events using the relatively short half-life predicted in [37]. The result in terms of sensitivities to the $0 \nu \beta \beta$ half-life and neutrino masses are shown in Table 2.3 together with the present best data. The loss in efficiency due to the asymmetric cut and to the tails beyond $2 \sigma$ are taken into account appropriately.

With the aggressive 100 ton yr measurement, the EXO experiment will be sensitive to $\sim .010 \mathrm{meV}$ Majorana neutrino mass. Within this context, the purpose of this thesis is to study the trapping and spectroscopy of Ba-II in the presence of xenon gas. 


\section{Chapter 3}

\section{Laser Induced Fluorescence}

EXO uses Laser Induced Fluorescence (LIF) spectroscopy as a diagnostic tool to observe and counted trapped barium ions. The interaction of electromagnetic radiation with atomic systems is an enormously rich and diverse field that is much too broad to cover in detail here. The material summarized in this chapter and further discussions can be found in many standard textbooks. For instance, the interaction between laser radiation and atoms is discussed in the framework of time-dependent perturbation theory in the standard quantum mechanics textbooks by Liboff [38] and Sakurai [39]. Several textbooks focused on lasers contain relevant sections, such as those by Yariv [40] and Svelto [41]. For spectroscopy, the text by Cowan [42] is indispensable. Finally, for a theoretical perspective, the work of Cohen-Tannoudji, e.g. Ref. [43], should be consulted.

In keeping with the "tool" status of LIF as used in the EXO barium trap, this chapter will briefly survey the theory of barium ion fluorescence. The discussion will start with two level systems, followed by collisional and Doppler broadening. Ba-II includes a meta-stable third level which significantly complicates the spectroscopy relative to two-level systems. In order to describe the full multi-level phenomenology, the Optical Bloch Equations (OBE) will be presented in the final section. 


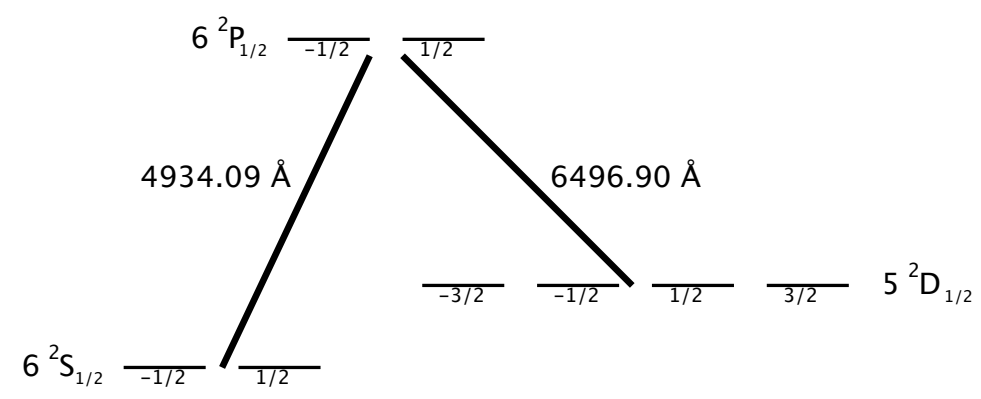

Figure 3.1: The electronic levels of Ba-II. The magnetic sublevels of each state are shown along with the associated laser wavelengths. The metastable $5^{2} D_{3 / 2}$ state has an $\sim 80$ second lifetime.

\subsection{Ba-II spectroscopy}

Singly ionized barium, Ba-II, has been very well studied in ion traps as a candidate system for optical clocks $[6,44,45]$. Ba-II has a single electron outside of a closed shell of inner electrons and behaves very much like the hydrogenic alkali earth elements of the first column of the periodic table. The lowest three electronic levels of Ba-II are shown in Fig. 3.1, complete with the magnetic fine state sub-levels and associated transition wavelengths in vacuum. An excited ion in the $6^{2} P_{1 / 2}$ state will transition to the metastable $5^{2} D_{3 / 2}$ state with $25 \%$ probability. The metastable state has a $79.8 \pm 4.6 \mathrm{~s}$ lifetime [45], so fluorescence experiments require both an excitation laser at $493 \mathrm{~nm}$ and a "clean-up" or repump laser at $650 \mathrm{~nm}$ to prevent optical pumping into the metastable "dark" state. It should be noted that These properties apply to barium ions in vacuum and may not necessarily apply to barium ions in gas.

\subsection{Two level systems}

Consider an atom with two electronic states, $|1\rangle$ and $|2\rangle$, with energies, $E_{1}$ and $E_{2}$, as shown in Fig. 3.2. Three possible interactions can occur between the atom and a photon of energy, $\hbar \omega_{12}=E_{2}-E_{1}$. First, an atom in the excited state, $|2\rangle$, can spontaneously decay to the ground state, $|1\rangle$, emitting a photon into $4 \pi$ as shown in 
Fig. 3.2(A). Second, an atom in the ground state can absorb a photon and transition to the excited state as shown in Fig. 3.2(B). Finally, in the presence of one or more incident photons of $\hbar \omega_{12}$, an excited atom can undergo stimulated emission and emit a photon into the same mode as the incident photon(s) as shown in Fig. 3.2(C). The stimulated emission photon is indistinguishable from the incident photons. In LIF experiments, a laser source pumps the atom to the excited state so that spontaneous emission into $4 \pi$ can occur and be observed as in Fig. 3.2(A). If the pumping laser is too powerful, then stimulated emission dominates and the re-emitted photons cannot be seen.

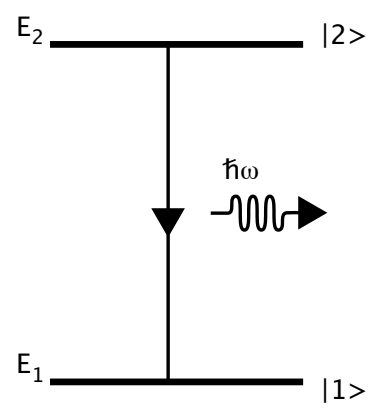

(A)

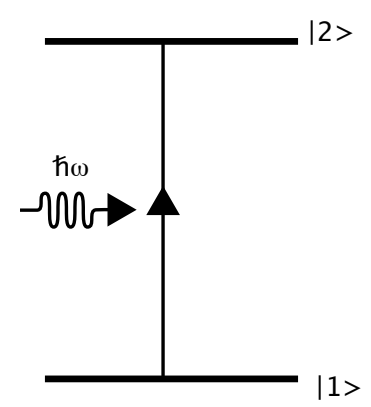

(B)

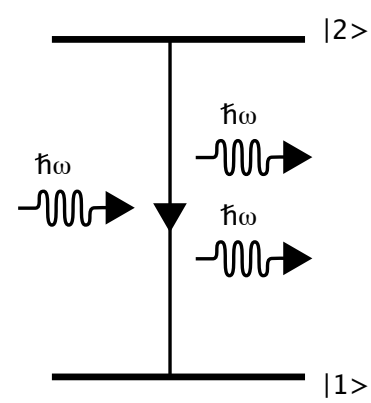

(C)

Figure 3.2: A two level system with $E_{2}>E_{1}$ interacting with a photon of energy $E=\hbar \omega_{12}=E_{2}-E_{1}$. (A) Spontaneous emission, (B) absorption, and (C) stimulated emission.

We are most interested in the behavior of an atom exposed to the harmonic perturbation of a coherent, monochromatic electromagnetic wave such as that produced by a laser. Standard quantum mechanics textbooks treat this situation in some detail, so we restrict ourselves to summarizing the approximations and results. The semi-classical approach used here follows Liboff $\$ 13.9$ [38]. Given an atom and an electromagnetic vector potential $\vec{A}$, the complete Hamiltonian is given by,

$$
\begin{aligned}
\mathbf{H} & =\frac{1}{2 m}\left[\vec{p}-\frac{e}{c} \vec{A}(r, t)\right]^{2}+V(r) ; \\
\mathbf{H}_{0}=\frac{\vec{p}^{2}}{2 m}+V ; \quad \mathbf{H}^{\prime} & =-\frac{e}{2 m c}[\vec{p} \cdot \vec{A}+\vec{A} \cdot \vec{p}] ; \quad \mathbf{H}^{\prime \prime}=\frac{e^{2}}{2 m c^{2}} \vec{A}^{2} ;
\end{aligned}
$$


where $V(r)$ is the electric potential. $\mathbf{H}_{0}$ is the Hamiltonian of the unperturbed electron, $\mathbf{H}^{\prime}$ is treated as a perturbation, and $\mathbf{H}^{\prime \prime}$ is neglected in the weak field limit. For an incident plane wave of frequency, $\omega$, propagation vector, $\vec{k}$, and polarization, $\boldsymbol{\epsilon}$, the vector potential is $A(r, t)=\boldsymbol{\epsilon} A_{0} \cos (\vec{k} \cdot \vec{r}-\omega t)$. In the steady state, the electromagnetic perturbation couples between the atomic energy levels $|1\rangle$ and $|2\rangle, E_{2}>E_{1}$ :

$$
\left\langle 2\left|\mathbf{H}_{ \pm}\right| 1\right\rangle=-\frac{e}{m}\left(\frac{2 \pi \hbar}{\omega}\right)^{1 / 2}\left\langle 2\left|\boldsymbol{\epsilon} \cdot \vec{p} e^{ \pm i \vec{k} \cdot \vec{r}}\right| 1\right\rangle
$$

Here, $\mathbf{H}_{ \pm}$, are the $\vec{p} \cdot \vec{A}$ and the $\vec{A} \cdot \vec{p}$ portions of $\mathbf{H}^{\prime}$ responsible for absorption and stimulated emission, respectively. The harmonic perturbation allows transitions between energy levels via the operator, $\boldsymbol{\epsilon} \cdot \vec{p} e^{ \pm i \vec{k} \cdot \vec{r}}$. In the dipole approximation, the term $e^{i \vec{k} \cdot \vec{r}}=1+i \vec{k} \cdot \vec{r}+\ldots$ is approximated by the leading term. This leads to the final result,

$$
\left\langle 2\left|\mathbf{H}_{+}\right| 1\right\rangle=\left\langle 1\left|\mathbf{H}_{-}\right| 2\right\rangle=i e(2 \pi \hbar \omega)^{1 / 2}\langle 2|\boldsymbol{\epsilon} \cdot \vec{r}| 1\rangle,
$$

with an associated transition rate

$$
w_{1 \rightarrow 2}=\frac{2 \pi}{\hbar} \frac{e^{2}}{m_{e}^{2} c^{2}}\left|A_{0}\right|^{2}|\langle 2|\epsilon \cdot \vec{r}| 1\rangle|^{2} \delta\left(E_{2}-E_{1}-\hbar \omega\right) .
$$

The transition rate in Eq. 3.5 reflects several fundamental aspects of atom-photon interactions. First, the excitation probability from $2 \rightarrow 1$ is the same as the probability to transition from $1 \rightarrow 2$. In matrix representation,

$$
H_{1,2}=H_{2,1}^{*}=i e(2 \pi \hbar \omega)^{1 / 2}\langle 2|\boldsymbol{\epsilon} \cdot \vec{r}| 1\rangle
$$

Second, the operator $\vec{r}$ is a rank one spherical tensor $Y_{l}^{m}(\theta, \phi)$ with $l=1$ and $m=$ $-1,0,1$. In order for the matrix element, $\langle 2|\vec{r}| 1\rangle$, to be non-zero, the states must satisfy the dipole selection rules,

$$
\begin{aligned}
\Delta l & =l^{\prime}-l= \pm 1 \\
\Delta m & =m^{\prime}-m=0, \pm 1 .
\end{aligned}
$$


Note that these selection rules are not absolute since they depend on the dipole approximation. Other transitions do occur, but with substantially lower probability. Third, the transition rate is proportional to the incident laser power, $\left|A_{0}\right|^{2}$. Finally, the $\delta$-function in Eq. 3.5 ensures energy conservation when a photon is absorbed or emitted by requiring the photon energy to be equal to the energy splitting.

\subsection{Collisonal line broadening}

The $\delta$-function of Eq. 3.5 assumes infinitely narrow energy levels, an unphysical supposition. In reality, the excited state has a finite spontaneous decay lifetime, $\tau_{s p}$, and Lorentzian frequency response. To see why the response is Lorentzian, consider the atom as a classical dipole oscillator with exponential decay rate, $\Gamma_{s p}=1 / \tau_{s p}$. In the time domain, the amplitude for an oscillator of frequency $\omega_{0}$ is

$$
E(t)=E_{0} \cos \omega_{0} t e^{-\Gamma_{s p} t} .
$$

We take the Fourier transform of $E(t)$ to find the frequency response,

$$
\begin{aligned}
\bar{E}(\omega) & =\int_{0}^{\infty} E_{0} \cos \omega_{0} t e^{-\Gamma_{s p} t} e^{-i \omega t} d t \\
& =\frac{E_{0}}{2}\left[\frac{1}{\Gamma_{s p}-i\left(\omega_{0}-\omega\right)}+\frac{1}{\Gamma_{s p}-i\left(\omega_{0}+\omega\right)}\right] .
\end{aligned}
$$

In the rotating wave approximation, $\omega \sim \omega_{0}$, and the second term may be neglected. The frequency response is then

$$
\bar{E}(\omega)=\frac{E_{0} / 2}{\Gamma_{s p}-i\left(\omega_{0}-\omega\right)},
$$

with the normalized Lorentzian power spectrum

$$
g(\omega) \propto|E(\omega)|^{2} \equiv \frac{\Gamma_{s p} / \pi}{\left(\omega-\omega_{0}\right)^{2}+\Gamma_{s p}^{2}},
$$




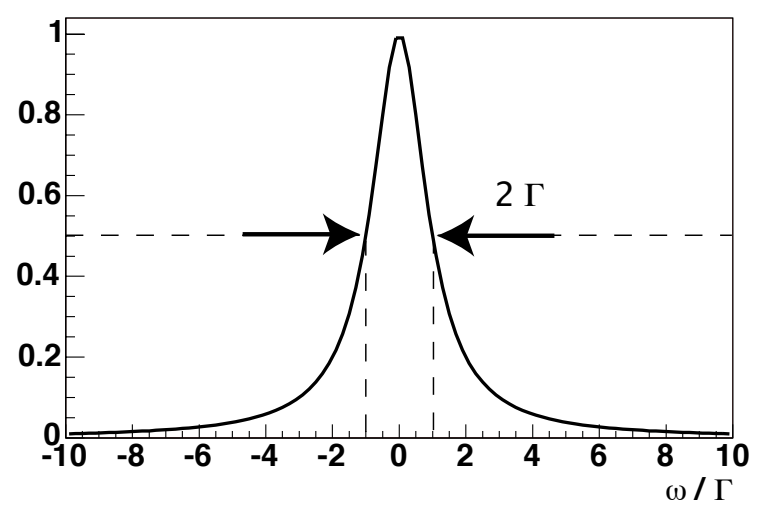

Figure 3.3: The Lorentzian frequency response of a damped harmonic oscillator. The FWHM is $2 \Gamma_{s p}$.

where $\int g(\omega) d \omega=1$. The power spectrum has a Full-Width, Half-Maximum (FWHM) of $2 \Gamma_{s p}$ and is shown in Fig. 3.3. Although this derivation is based upon a classical oscillator model, more complete quantum derivations yield the same fundamental result: an oscillator with exponential time dependence has a Lorentzian frequency response.

One line-broadening mechanism of particular interest for EXO is collisional broadening. Bellert et al. [46] discuss the interaction potential between atoms and noble gases in detail. In this section, we present a simplified discussion sufficient to introduce collisional broadening. At long range, the dominant interaction between Ba-II and a noble buffer gas atom is the monopole-induced dipole interaction potential,

$$
V_{i}(\vec{r}) \approx-\frac{\alpha_{g} e^{2}}{2 \vec{r}^{4}}+A_{i} e^{-b_{i} r}
$$

where $\alpha_{g}$ is the polarizability of the background gas, $\vec{r}$ is the distance between the two atoms, and $i$ indexes the electronic state of the Ba-II ion [46, 47]. The exponential term, $A_{i} e^{-b_{i} R}$, is a standard parameterization of the short range electronic repulsion which depends on the ion's electronic state. Although higher order terms are neglected in Eq. 3.12, Bellert et al. show that the terms give rise to a difference in the interaction depending on the ionic state and must be included to quantitatively model 


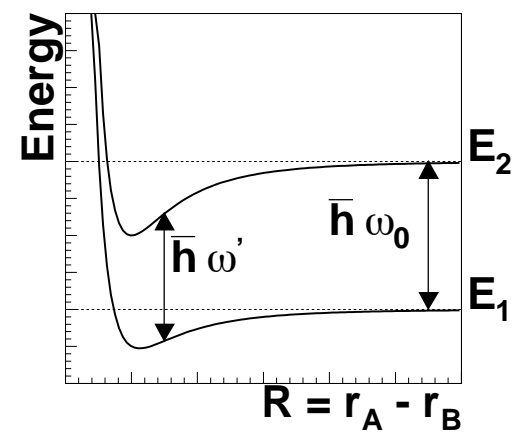

(a) Interaction potential

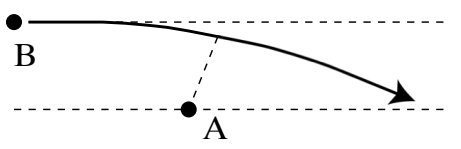

(b) Collision

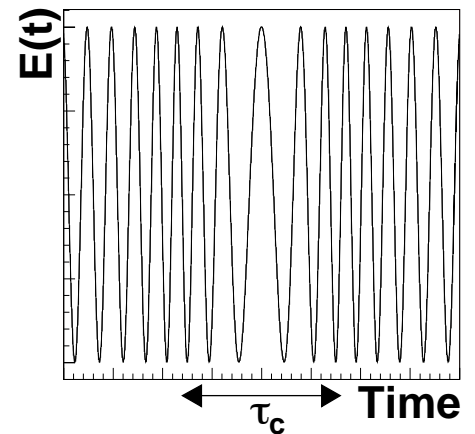

(c) Frequency chirp

Figure 3.4: Effect of a collision on an atomic oscillator.

the available data. For our purposes, a detailed model of the interaction potential is unnecessary; it suffices to recognize that a) at long ranges, the potential varies as $\alpha_{g} / r^{4}$ and b) the potential depends on the electronic state of the ion.

The radial dependence of the interaction potential is schematically plotted for both the ground and excited states of an ion in Fig. 3.4(a). In addition to exerting a force on the particles and changing their trajectory as shown in Fig. 3.4(b), the collision changes the energy separation of the electron levels from $\hbar \omega_{0}$ to $\hbar \omega^{\prime}$, resulting in a frequency chirp for the duration of the collision, $\tau_{c}$, as shown in Fig. 3.4(c).

To estimate the effect of the frequency chirp, consider the phase change during a collision with constant velocity, $v$, closest approach, $R_{0}$, and ignore any changes in trajectory. Then for the potential of Eq. 3.12, the change in oscillator phase is

$$
\begin{aligned}
\Delta \phi\left(R_{0}\right) & =\int_{-\infty}^{\infty} \Delta \omega d t \\
& \approx \frac{1}{v \hbar} \int_{-\infty}^{\infty} \frac{\alpha_{g} e^{2}}{2 \vec{r}^{4}}+A_{i} e^{-b_{i} r} d r
\end{aligned}
$$

For values of $\Delta \phi>1$, the phase after the collision is essentially unrelated to the phase before the collision. We define a depolarization cross-section, $\sigma_{\phi}$, for collisions which randomize the phase. Consider a mean time between collisions of $t_{c}=1 / n \sigma_{\phi} \bar{v}$, where $n$ is the density of background gas and $\bar{v}$ is the average relative velocity. Assuming the duration of the collisions is much shorter than the time between collisions, $\tau_{c} \ll$ 
$t_{c}$, the collisions can be considered as discrete perturbations. Since the collisions happen at constant average rate, the distribution of the interval between collisions has an exponential dependence. This situation is similar to the exponential interval distribution found for spontaneous decay, and the derivation leading to Eq. 3.11 applies. Consequently, the linewidth of the excited state is no longer determined by the spontaneous emission rate alone but also by the collision rate, giving a linewidth

$$
\Gamma=\Gamma_{s p}+n \sigma_{\phi} \bar{v}=\Gamma_{s p}+\Gamma_{c}
$$

The unperturbed power spectrum of Eq. 3.11 is modified to,

$$
g_{c}(\omega)=\frac{\left(\Gamma+\Gamma_{c}\right) / \pi}{\left(\omega-\omega_{0}+\Delta \omega\right)^{2}+\left(\Gamma+\Gamma_{c}\right)^{2}},
$$

where $\Delta \omega$ is the line shift. The line shift results from the cumulative effect of many long-range collisions which slightly shifts the atomic level splitting from the vacuum value.

A primary goal of this research is to measure the pressure broadening of Ba-II in Xe to pressures relevant for the EXO detector. Unfortunately, some difficulty lies in predicting the cross section required to dephase the atomic dipole and hence the magnitude of the pressure broadening. Rather than calculate the cross-sections from first principles, we can use measurements of similar systems found in the literature for an order of magnitude estimate. Here we list the results from experiments with similar atomic species (Cs, Ba-I, Ba-II) and from similar background gases (Ar, Xe). Cesium has the same electronic configuration as Ba-II, so we expect the collision broadening to be similar to that of Cs-Xe collisions, for which the broadening is $\Gamma_{c} \sim 11 \mathrm{MHz} /$ torr and the level shift is $-8 \mathrm{MHz} /$ torr [48]. Neutral barium's electronic configuration also shares features with Ba-II. Ehrlacher et al. measure a broadening rate coefficient for neutral $\mathrm{Ba}$ in $\mathrm{Ar}, k_{b r}$, of $2.35 \times 10^{-9} \mathrm{~cm}^{3} / \mathrm{s}$ at $810 \mathrm{~K}$ [49]. At room temperature, this broadening rate is equivalent to $\Gamma_{c} \sim 18.6 \mathrm{MHz} /$ torr and the corresponding level shift is $-5.9 \mathrm{MHz} /$ torr [49]. The only measurement we found in the literature of Ba-II broadening is due to Gallagher, who used the zero-field crossing technique to measure 
$\mathrm{Ba}-\mathrm{II}$ in $\mathrm{Ar}$ at $413 \mathrm{~K}$ and 1 torr. He placed a limit on the depolarization cross section

of $\sigma_{B a A r}=0.0 \pm 0.25 \times 10^{-14} \mathrm{~cm}^{2}$ [50]. At room temperature, this cross section places a limit on the collisional broadening of $0 \pm 87 \mathrm{MHz} /$ torr. Taking the range of these empirical data and adjusting for the high polarizability of Xe, we guess that the collisional broadening of Ba-II in Xe could be as low as $10 \mathrm{MHz} /$ torr and no higher than $100 \mathrm{MHz} /$ torr.

\subsection{Doppler broadening}

Both the spontaneous emission rate and collisional broadening act equally on all atoms at all times and are categorized homogeneous broadening. In contrast, inhomogeneous broadening is an increase in the linewidth which results from a time or position dependence of the fluorescence rate across an ensemble of ions. For instance, Doppler broadening is an inhomogeneous broadening mechanism originating from the motion of the observed ions. For non-relativistic speeds, an atom traveling at velocity, $v$, (anti)parallel to an electromagnetic wave of frequency, $f$, "sees" a frequency in the atomic rest frame shifted by,

$$
\Delta f=\frac{v}{c} f .
$$

Of course, if the atom moves at constant velocity, then the absorption line center is shifted but not broadened by the Doppler shift. Similarly, an atom moving perpendicular to the electromagnetic field experiences no Doppler shift at all. Two types of measurements give rise to inhomogeneous Doppler broadening. An instantaneous measurement of an ensemble of atoms, each of which is moving at a different velocity, yields a Doppler broadened spectrum corresponding to the velocity distribution of the atoms. Conversely, a long measurement of a single atom moving at a variety of velocities also yields a Doppler broadened spectrum reflecting the atom's motion.

There are two major contributions to Doppler broadening in trapped Ba-II spectroscopy; thermal broadening and micromotion broadening. Thermal broadening is common to many experiments, and results from measurements of atoms in thermal equilibrium with the environment. Ions colliding with a buffer gas are in thermal 
equilibrium and have a Maxwell-Boltzmann velocity distribution,

$$
P(\vec{v}) d^{3} v=\left(\frac{m}{2 \pi k T}\right)^{3 / 2} \exp \left(-\frac{m \vec{v}^{2}}{2 k T}\right) d^{3} \vec{v}
$$

where $P(\vec{v}) d^{3} \vec{v}$ is the probability that an atom has a velocity between $\vec{v}$ and $\vec{v}+d^{3} \vec{v}$. The distribution is a Gaussian with a $1 / e$ half-width, $\sigma_{\omega_{D}}=\sqrt{k T / m}$. For barium, the width of the thermal velocity distribution at $300 \mathrm{~K}$ is $\sigma_{\omega_{D}}=135 \mathrm{~m} / \mathrm{s}$. Note that $\vec{v}$ is a vector quantity with $\vec{v}^{2}=v_{x}^{2}+v_{y}^{2}+v_{z}^{2}$, where $x, y, z$ are three orthogonal axes. Since the laser absorption is measured along a single axis, we are most interested in the distribution along one axis,

$$
P\left(v_{x}\right) d v_{x}=\iint P(\vec{v}) d v_{y} d v_{z}=\sqrt{\frac{m}{2 \pi k T}} \exp \left(-\frac{m v_{x}^{2}}{2 k T}\right) .
$$

Combining the Doppler shift of Eq. 3.16 with Eq. 3.18, we calculate a Doppler frequency profile for thermal atoms,

$$
P(\Delta f) d \Delta f=\sqrt{\frac{m c^{2}}{2 \pi k T f^{2}}} \exp \left(-\frac{m c^{2} \Delta f^{2}}{2 k T f^{2}}\right),
$$

which yields a Doppler broadened linewidth,

$$
\sigma \omega_{D}=\sqrt{\frac{k T}{m \lambda^{2}}}
$$

where $\lambda=c / f$ is the wavelength of the electromagnetic wave. This Gaussian Doppler line differs substantially from the Lorentzian line defined in Eq. 3.11; the two lineshapes are shown in Fig. 3.5 with equal widths. In particular, the Lorentzian distribution has substantial "wings" at low and high frequency that are absent in the Gaussian distribution. As we will show in later sections, ions confined in an RF trap in the presence of a buffer gas do not have a perfect thermal velocity distribution, and in fact, measurements of the Doppler broadened profile reveal the trapped ion kinematics.

In addition, ions in an RF trap experience a unique form of Doppler broadening 


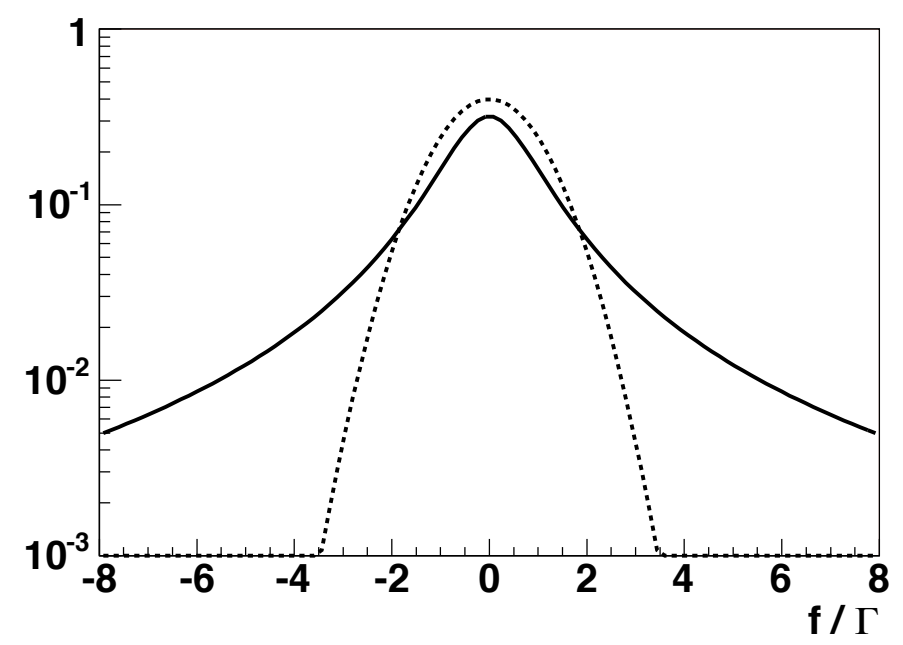

Figure 3.5: The Lorentzian (solid line) and Gaussian (dashed line) distributions plotted together with $\sigma=\Gamma=1$. The crossover between the distributions occurs at $f / \Gamma \sim 1.85$.

resulting from the ion's micromotion. The details of the ion motion will be discussed in great detail in the following chapters. For the purposes of spectroscopy, we will now assume without proof that the ion oscillates at frequency $\Omega$ with maximum velocity $v_{0}$. Here, we follow the derivation of Blümel, et al. [51]. In the rest frame of the ion, the spatial oscillation corresponds to a frequency modulation of the electromagnetic wave such that the Lorentzian with line center $\omega_{0}$ develops sidebands at the frequencies $\omega_{n}=\omega_{0} \pm n \Omega$ where $n=0,1,2, \ldots$ The absorption at frequency, $\Delta \omega=\omega-\omega_{0}$, is given by the sum of the sideband absorption,

$$
I(\Delta \omega)=\sum_{n=-\infty}^{\infty} \frac{J_{n}^{2}(\beta)}{(\Delta \omega+n \Omega)^{2}+\Gamma^{2}},
$$

where the $J_{n}$ are Bessel functions with modulation depth

$$
\beta=\frac{k \cdot v_{0}}{\Omega} .
$$

Fig. 3.6 shows the effect of the frequency modulation on the absorption profile. The 


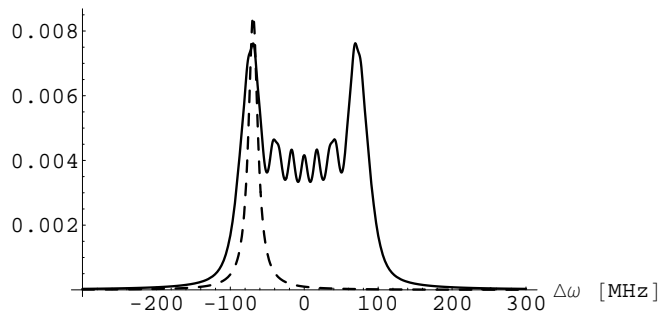

(a) $\beta=10$

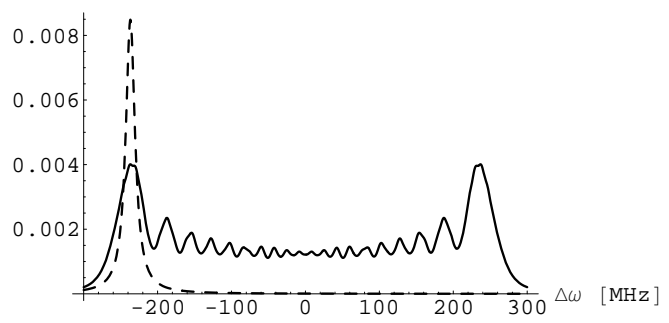

(b) $\beta=30$

Figure 3.6: The absorption spectrum for a sinusoidally oscillating atom with $\beta=10$ and $\beta=30$. The unmodulated spectrum is shown with the dashed line, shifted to coincide with the peak of the modulated spectrum, which is shown with the solid line. $\Omega=8.5 \mathrm{MHz}$.

dashed line is the original Lorentzian distribution with $\Gamma=15 \mathrm{MHz}$, similar to the BaII linewidth, and the solid line is the frequency modulated distribution with $\Omega=8.5$ $\mathrm{MHz}$, the trap oscillation frequency. The modulation spectrum is shown with (a) $\beta=10$ and (b) $\beta=30$. Notice that the frequency modulation splits and shifts the peak signal from the vacuum value, broadening the line and reducing the peak intensity. The modulation depth can be measured from the shift of the resonance peak from the unmodulated value.

The combined effect of these broadening mechanisms on Ba-II spectroscopy requires numerical analysis using the Optical Bloch Equations discussed in §3.5. In the interest of comparing the relative impact of the broadening mechanisms, we tabulate the numeric values of spontaneous emission, collisional broadening, thermal broadening, and modulation for both the red and blue transitions in Table 3.1. Together, the principal effect of these broadening mechanisms on EXO single ion spectroscopy is to shift the peak signal and reduce the available signal.

\subsection{Optical Bloch Equations}

The two level phenomenology developed in $\S 3.2$ does not directly apply to Ba-II spectroscopy because of the metastable $D$-state shown in Fig. 3.1. To describe the 


\begin{tabular}{l|cc}
\hline Source & Blue width & Red width \\
\hline \hline Spontaneous emission & $15.2 \mathrm{MHz}$ & $5.28 \mathrm{MHz}$ \\
Collisional broadening $(1 \mathrm{torr})$ & $\simeq 50 \mathrm{MHz} \simeq \simeq 50 \mathrm{MHz}$ \\
Collisional broadening $(1 \mathrm{~atm})$ & $\simeq 3800 \mathrm{MHz} \simeq 3800 \mathrm{MHz}$ \\
Thermal Doppler width $(300 \mathrm{~K})$ & $280 \mathrm{MHz}$ & $210 \mathrm{MHz}$ \\
Modulated width $(\beta=10, \Omega=8.5 \mathrm{MHz})$ & $85 \mathrm{MHz}$ & $75 \mathrm{MHz}$ \\
Modulated width $(\beta=30, \Omega=8.5 \mathrm{MHz})$ & $252 \mathrm{MHz}$ & $242 \mathrm{MHz}$
\end{tabular}

Table 3.1: Contributions to the blue and red absorption linewidths.

phenomenology of Ba-II absorption and fluorescence, at least the three electronic states, $6^{2} S_{1 / 2}, 6^{2} P_{1 / 2}$ and $5^{2} D_{3 / 2}$, must be considered. For completeness, all 8 magnetic sublevels and 4 laser polarizations (two each for the red and blue lasers) should be included in calculations of the population and coherences of all 8 states. In this section, we will summarize the mathematical machinery of density matrices and the Optical Bloch Equations (OBE) used to calculate multi-level atomic systems.

The hardware and mathematics required to work with three level atoms are more complicated than those presented previously for two level systems. However, the requirement that two lasers be resonant in order to generate a fluorescence signal significantly improves the Ba-II tagging, eliminating the possibility that an atom other than barium produces a positive signal. In addition, there are several techniques that take advantage of barium's multi-level electronic structure to reduce signal to noise. The major source of noise in LIF measurements is scattered photons from the input lasers, so we choose our techniques to keep the number of background photons small and constant during the measurement. The simplest technique is to compare the signal when both lasers are on and off resonance. Slightly more complicated is to compare the number of blue photons while the red laser is turned on and off by using a blue-pass filter. With the red laser off, the ions are optically pumped into the metastable $5 D_{3 / 2}$ and the ions go dark until the red laser is turned back on. In this case, the signal to noise ratio is determined by the number of background blue photons. The most complicated technique eliminates the blue background by "shelving" the ions in the metastable $5 D_{3 / 2}$ using an appropriate blue laser pulse, then pumping the ion back to the $6 S_{1 / 2}$ using a red laser pulse. When relaxing to 
the ground state, the atom emits a blue photon while the blue laser is off, allowing a measurement of the ion state with near zero background.

To develop an appropriate theory, we summarize the density matrix formulation of quantum mechanics using the derivation in Liboff [38]. We begin by defining the density matrix operator, $\rho$, for a system in state $\psi$ as

$$
\rho_{i j}=\langle i \mid \psi\rangle^{*}\langle j \mid \psi\rangle=a_{i}^{*} a_{j},
$$

where the $|i, j\rangle$ are the electronic states of the system and $a_{i}$ are the coefficients of the projection of $\psi$ onto the basis states. The expectation value of an operator $A$ is given by

$$
\langle A\rangle=\sum_{i} \sum_{j}\langle\psi \mid i\rangle\langle i|A| j\rangle\langle j \mid \psi\rangle=\operatorname{Tr} \rho A .
$$

The probability of measuring the system in the state $|i\rangle$ is

$$
P_{i}=|\langle\psi \mid i\rangle|^{2}=\rho_{i i},
$$

and the equation of motion for the density matrix is,

$$
i \hbar \frac{\partial \rho}{\partial t}=[H, \rho] .
$$

As expected, the time evolution of the density matrix depends on the Hamiltonian, $H$, of the system. If the Hamiltonian is diagonal, there is no coupling between the states $|i\rangle$ and the system is stationary; the probabilities are constant. Off diagonal elements couple the levels and allow the atomic population to spread among the states.

In order to simplify the mathematics, we are free to transform the state vector, $\left|\psi_{i}\right\rangle$, by a unitary matrix, $U_{i j}$, such that $\left|\psi_{j}^{\prime}\right\rangle=\sum_{i} U_{i j}\left|\psi_{i}\right\rangle$. Dropping the subscripts for simplicity, we observe that in the rotated frame, $\rho^{\prime}=U \cdot \rho \cdot U^{-1}$. To maintain the validity of the equation of motion, Eq. 3.26, the Hamiltonian must be transformed as well,

$$
H^{\prime}=U H U^{-1}-i \hbar U \cdot \frac{\partial U^{-1}}{\partial t} .
$$

In practice, we choose the rotations, $U$, which transform the Hamiltonian, $H^{\prime}$, into a 
time-independent form.

We now consider the Hamiltonian for Ba-II, taking the $6^{2} S_{1 / 2}$ ground state, the $6^{2} P_{1 / 2}$ excited state, and the $5^{2} D_{3 / 2}$ metastable state to be the levels, $|0\rangle,|1\rangle,|2\rangle$, respectively, which we arrange in a state vector,

$$
\psi=\left(\begin{array}{c}
|0\rangle \\
|1\rangle \\
|2\rangle
\end{array}\right)
$$

Restoring the time-dependence to the off-diagonal Hamiltonian terms from Eq. 3.6 and combining with the states' unperturbed energies, $\hbar \omega_{0,1,2}$, the three level Hamiltonian is

$$
H=\left(\begin{array}{ccc}
\hbar \omega_{0} & \hbar \Omega_{B} e^{i \omega_{B} t} & 0 \\
\hbar \Omega_{B}^{*} e^{-i \omega_{B} t} & \hbar \omega_{1} & \hbar \Omega_{R} e^{-i \omega_{R} t} \\
0 & \hbar \Omega_{R}^{*} e^{i \omega_{R} t} & \hbar \omega_{2}
\end{array}\right) .
$$

Here, $\Omega_{B(R)}$, are the blue(red) Rabi frequencies which measure the strength of the off diagonal coupling and are proportional to the dipole matrix element in Eq. 3.6. $\omega_{B(R)}$, are the blue(red) laser frequencies. Note that the sign of the off-diagonal exponentials, $\exp [ \pm i \omega t]$, corresponds to photon absorption and emission, respectively. We perform two unitary transformations; the first to the interaction picture, $U_{I}$, and the second to the rotating frame, $U_{R}$,

$$
U_{I}=\left(\begin{array}{ccc}
e^{i \omega_{0} t} & 0 & 0 \\
0 & e^{i \omega_{1} t} & 0 \\
0 & 0 & e^{i \omega_{2} t}
\end{array}\right), \quad U_{R}=\left(\begin{array}{ccc}
1 & 0 & 0 \\
0 & e^{i \Delta \omega_{b} t} & 0 \\
0 & 0 & e^{i\left(\Delta \omega_{b}-\Delta \omega_{r}\right) t}
\end{array}\right)
$$

The blue detuning, $\Delta \omega_{b}$, is equal to the difference between the laser frequency and the level splitting, $\Delta \omega_{b}=\omega_{b}-\left(\omega_{1}-\omega_{0}\right)$, and the red detuning is defined similarly. 
Under these transformations, the Hamiltonian is transformed to

$$
H^{\prime}=\hbar\left(\begin{array}{ccc}
0 & \Omega_{b} & 0 \\
\Omega_{b}^{*} & -\Delta \omega_{b} & \Omega_{r} \\
0 & \Omega_{r}^{*} & -\left(\Delta \omega_{b}-\Delta \omega_{r}\right)
\end{array}\right) \text {. }
$$

The net effect of the transformations, $U_{I}, U_{R}$, is to transfer the time dependence of the Hamiltonian to the state vectors, leaving a Hamiltonian that only depends on the Rabi frequencies and the detunings. Since there are no off-diagonal elements in our unitary transformation matrices, the probability that the atom is in the state $|i\rangle$ is still given by the density matrix element, $\rho_{i i}$.

As discussed previously, the observed signal in the EXO ion trap is the blue spontaneous emission from the excited state to the ground state. Consequently, we are interested in calculating the population in the $P_{1 / 2}$ excited state. By applying Eq. 3.26 to the Hamiltonian Eq. 3.31 we find a differential equation for the excited state population,

$$
i \hbar \frac{\partial \rho_{11}}{\partial t}=\Omega_{B}^{*} \rho_{12}-\Omega_{B} \rho_{21}+\Omega_{R} \rho_{32}-\Omega_{R}^{*} \rho_{23}-\left(\Gamma_{b}+\Gamma_{r}\right) \rho_{11},
$$

where we have added the spontaneous decay term, $-\left(\Gamma_{b}+\Gamma_{r}\right) \rho_{11}$, by hand. In order to maintain the hermiticity of the density matrix and to conserve probability, we must add a spontaneous decay term to the other diagonal terms, $\rho_{00}^{s p}=\Gamma_{b} \rho_{11}, \rho_{22}^{s p}=\Gamma_{r} \rho_{22}$, as well as the off-diagonal terms, $\rho_{12}^{s p}=-\Gamma_{b} / 2 \rho_{11}$, etc. To calculate the population $\rho_{11}$ and the corresponding blue fluorescence rate, $\rho_{11} \Gamma_{b}$, the system of 9 differential equations, $d \rho_{i j} / d t$, must be solved simultaneously.

Rather than show the equation for $\rho_{11}$ here, we instead present graphs demonstrative of the observed Ba-II spectra for which $\Delta \omega_{b}$ is swept while $\Delta \omega_{r}$ is fixed at -25 MHz. The most notable feature of the three level OBEs, shown in Fig. 3.7(a), is the two photon resonance at $-25 \mathrm{MHz}$. When both the red and blue detunings are equal, ie. $\Delta \omega_{b}=\Delta \omega_{r}$, the coherences $\rho_{12}$ and $\rho_{23}$ destructively interfere and the population of the excited state goes to zero. In other words, the electron oscillates 


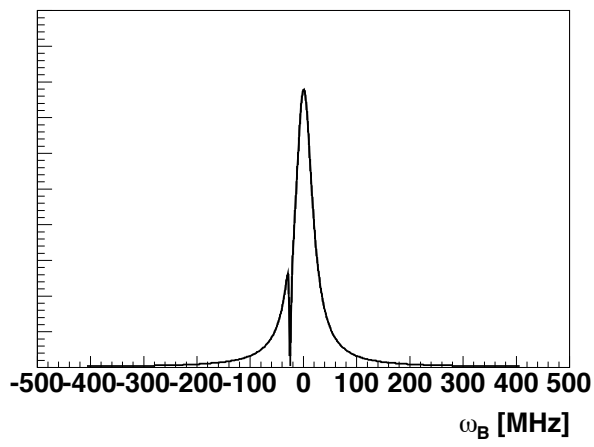

(a) $\Omega_{b}=\Omega_{r}=10 \mathrm{MHz}$

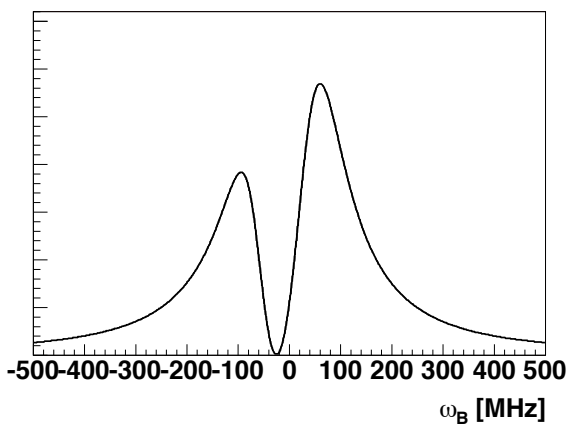

(b) $\Omega_{b}=100, \Omega_{r}=100 \mathrm{MHz}$

Figure 3.7: Blue laser spectra for Ba-II calculated from the three-level OBEs with fixed red detuning, $\Delta \omega_{r}=25 \mathrm{MHz}$. The y-axis normalization varies between plots for clarity.

directly from the ground state to the metastable state without populating the excited state. The laser power has a strong influence on the observed spectra, both by changing shape of the two photon resonance and by power broadening the linewidth and enlarging the two photon resonance as shown in Fig. 3.7(b).

With OBE calculations of the simplified 3-level and, when necessary, the full 8level Ba-II electronic structure, we can use laser spectroscopy to measure the ion environment within the RF trap. The Gaussian line-width of the fluorescence spectrum directly probes the temperature of the trapped ions, and the OBEs allow us to measure the collisional broadening at high pressures. Most importantly for this thesis, the fluorescence rate at constant laser frequency and power corresponds directly to the number of ions in the trap. 


\section{Chapter 4}

\section{The theory of single ion trapping}

Laser spectroscopy probes the electronic level structure of atoms with very high precision, limited only by the energy width of the levels themselves. Unfortunately, environmental factors often dominate the experimentally observed linewidth. The ideal spectroscopic system, rarely achieved, is a single atom at rest in vacuum, free from external fields and forces. Starting with the electrodynamic guiding of ions in a linear quadrupole mass filter first described by Paul in 1955 (c.f. Ref. [52]), decades of research in vacuum, laser and spectroscopic sciences culminated in the observation of a single, localized barium ion in 1979 [53]. This feat requires advanced laser spectroscopy, detailed understanding of the motion of a trapped ion, and ion cooling techniques. In the following sections, I will review electrodynamic ion trapping as applied in this experiment, beginning with the ideal behavior of a trapped ion and concluding with non-ideal phenomenology.

\subsection{Ideal traps}

The EXO spectroscopy experiment uses Radio Frequency (RF) traps to isolate barium ions in ultrahigh vacuum. The RF trapping scheme, first demonstrated by Straubel to trap oil droplets in 1955 [65] and by Wuerker et al. to trap charged aluminum particles in 1958 [54], uses a cylindrically symmetric quadratic electric potential to trap ions in free space. The quadratic form of the potential ensures a linear restoring force and 


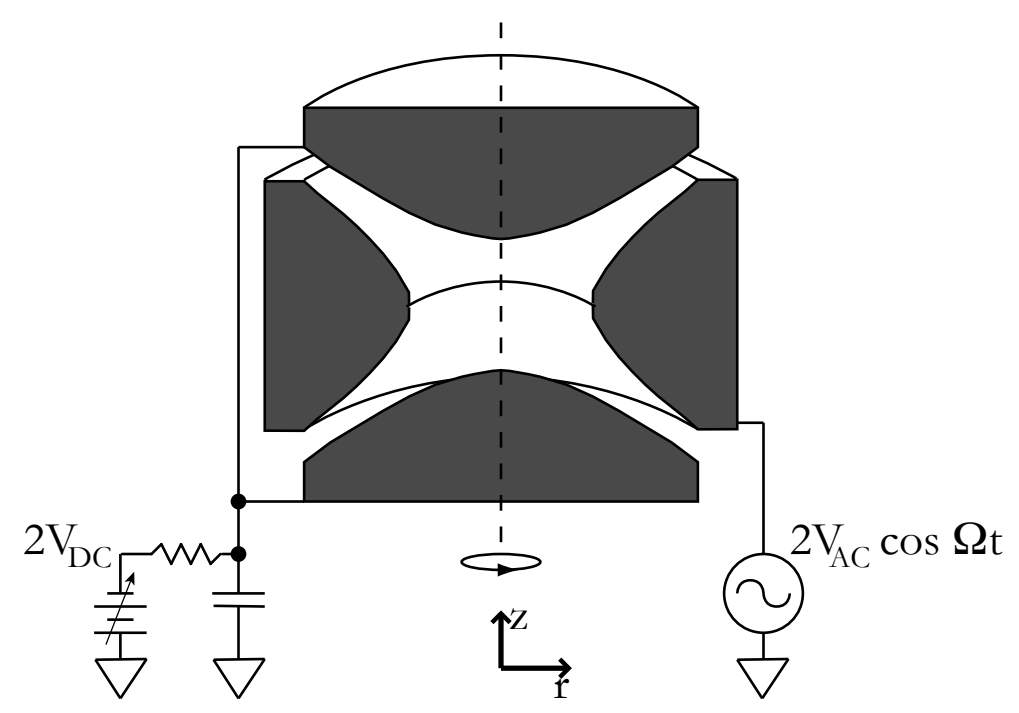

Figure 4.1: Schematic of a hyperbolic trap showing the applied DC and AC voltages.

thus a conservative potential. Of course, Laplace's equation in free space, $\nabla^{2} \Phi=0$, means an electric potential has no absolute minimum with which to localize a particle. In other words, in a static field a radial confinement must be accompanied by axial unloading and vice versa. If, however, the field oscillates in time, a stable situation exists in which the electric force changes sign more rapidly than the particle changes position. For certain parameters, the oscillating electric potential forms an effective static potential with an absolute minimum within which a particle can remain stably bound.

Consider an ideal quadrupole field formed by cylindrically symmetric, hyperbolic electrodes, as shown schematically in Fig. 4.1. In cylindrical coordinates, the potential is

$$
V(r, z, t)=\left[V_{D C}-V_{A C} \cos \Omega t\right]\left[\frac{z^{2}-r^{2} / 2}{z_{0}^{2}}\right],
$$

with endcap spacing, $2 \times z_{0}$, DC and $\mathrm{AC}$ voltages, $V_{D C}$ and $V_{A C}$, and $\mathrm{RF}$ oscillation frequency, $\Omega$. The origin in this coordinate system is the trap center; when connected as shown in Fig. 4.1 the applied voltages are twice the voltages in Eq. 4.1. A particle with charge $e$ and mass $m$ in the potential $V(r, z, t)$ experiences a force 
$\vec{F}=m d^{2} \vec{r} / d t^{2}=e \nabla V(r, z, t)$. The resulting equations of motion,

$$
\begin{gathered}
\frac{d^{2} z}{d t^{2}}=-2\left[\frac{e V_{D C}}{m z_{0}^{2}}-\frac{e V_{A C}}{m z_{0}^{2}} \cos \Omega t\right] z \\
\frac{d^{2} r}{d t^{2}}=\left[\frac{e V_{D C}}{m z_{0}^{2}}-\frac{e V_{A C}}{m z_{0}^{2}} \cos \Omega t\right] r
\end{gathered}
$$

are independent from each other and to a factor of -2 , identical. These equations are special instances of the Mathieu equation:

$$
\frac{d^{2} u_{i}}{d \tau^{2}}+\left(a_{i}-2 q_{i} \cos 2 \tau\right) u_{i}=0
$$

with the transformed parameters:

$$
\begin{aligned}
u_{i} & =r, z \\
\tau & =\Omega t / 2 \\
a_{z} & =-2 a_{r}=8\left(\frac{e V_{D C}}{m \Omega^{2} z_{0}^{2}}\right) \\
q_{z} & =-2 q_{r}=4\left(\frac{e V_{A C}}{m \Omega^{2} z_{0}^{2}}\right) .
\end{aligned}
$$

Since Mathieu first applied his eponymous equations to the modes of an elliptical vibrating membrane in 1868 , a sizeable literature has been devoted to their solutions and characteristics. The discussion here will summarize only those findings most relevant for ion trapping, following the treatment in Refs. [54] and [55]. The general solution of the Mathieu equation can be written in an infinite series,

$$
u_{i}(\tau)=A_{i} e^{\mu_{i} \tau} \sum_{n=-\infty}^{\infty} c_{i, 2 n} e^{2 n \tau i}+B_{i} e^{-\mu_{i} \tau} \sum_{n=-\infty}^{\infty} c_{i, 2 n} e^{-2 n \tau i}
$$

where $\mu_{i}, c_{i, 2 n}$ are functions of $a_{i}, q_{i}$, and $A_{i}, B_{i}$ are arbitrary constants determined by the initial conditions. The exponent, $\mu_{i}$, governs the dynamics of the solution. If $\mu_{i}$ has a real component, the positive exponential yields unbound orbits. Only if $\mu_{i}$ is purely imaginary, ie. $\mu_{i}=i \beta_{i}$, will particles be stably bound in oscillating orbits. 


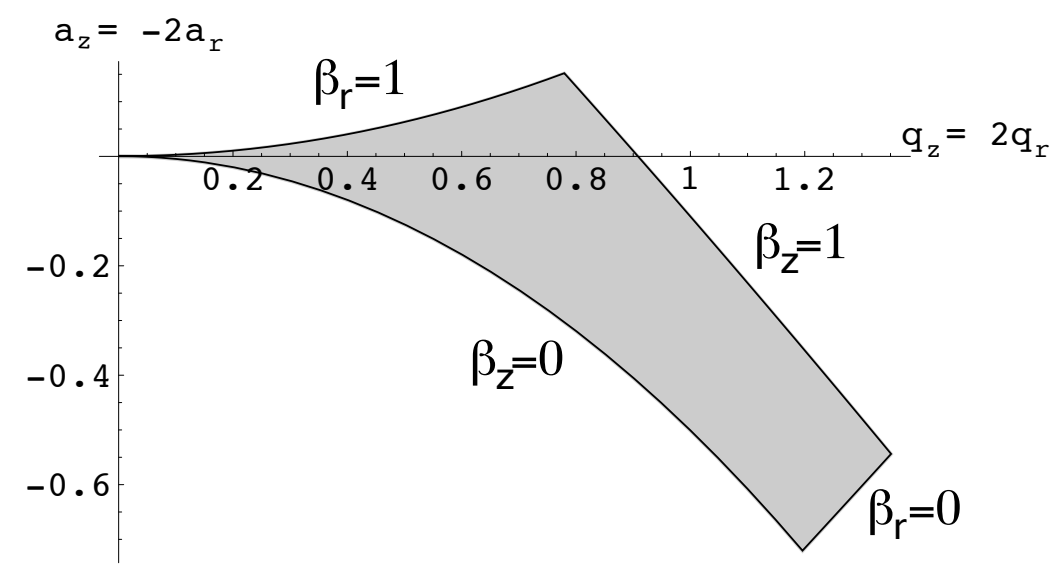

Figure 4.2: Single particle stability plot as a function of $q_{z}=-2 q_{r}=4 e V_{A C} /\left(m z_{0}^{2} \Omega^{2}\right)$ and $a_{z}=-2 a_{z}=8 e V_{D C} /\left(m z_{0}^{2} \Omega^{2}\right)$. When the $a, q$ lie within the grey region, particles are stably trapped.

When $\mu$ is pure imaginary, Eq. 4.5 simplifies to a more intuitive solution,

$$
u_{i}(\tau)=A_{i} \sum_{n=-\infty}^{\infty} c_{i, 2 n} \cos \left(\left(2 n+\beta_{i}\right) \tau+\phi_{i}\right)
$$

Following Ref. [55], we take $0<\beta_{i}<1$ for convenience. The $A_{i}$ and $\phi_{i}$ are determined by the initial conditions.

There are many combinations of $V_{A C}, V_{D C}$ and $\Omega$ for which the Mathieu equation has bound solutions; here we consider only the lowest voltage region for which $\left|a_{i}\right| \leq 1$. With this constraint, we can apply the equation of motion, Eq. 4.3, to the solution, Eq. 4.6, to get an infinite continued fraction solution for $a_{i}$ and $q_{i}$. Numerically, the solution is stable when $a_{i}$ and $q_{i}$ lie between the curves:

$$
\begin{aligned}
& a_{i}=-\frac{1}{2} q_{i}^{2}+\frac{1}{128} q_{i}^{4}-\frac{29}{2304} q_{i}^{6}+\frac{68687}{18874368} q_{i}^{8}+\ldots \quad \text { and } \\
& a_{i}=1-q_{i}-\frac{1}{8} q_{i}^{2}+\frac{1}{64} q_{i}^{3}-\frac{1}{1536} q_{i}^{4}-\frac{11}{35864} q_{i}^{5}+\ldots
\end{aligned}
$$

corresponding to $\beta_{i}=0$ and $\beta_{i}=1$, respectively. In Fig. 4.2, the dimensionless quantities $a_{z}$ and $q_{z}$ are plotted in place of the applied voltages $V_{A C}$ and $V_{D C}$. A 
particle is trapped only when $a_{z}$ and $q_{z}$ lie in the shaded region bound by the curves $\beta_{r}=\beta_{z}=0$ and $\beta_{r}=\beta_{z}=1$. For $V_{D C}=a_{i}=0$, the trap is only stable for values of $q_{z} \leq .908$.

Note that for the solution of the Mathieu equation in Eq. 4.6, there is no component of the particle motion at the drive frequency. In other words, the large electric fields oscillating at $\Omega$ do not add energy to the charged particles in the trap. Consider the power coupled into the particle's motion from the electric field. When averaged over one period of the RF oscillation, the power delivered to the particle is given by,

$$
\begin{aligned}
P & =\frac{d\langle W\rangle_{\Omega}}{d t} \\
& =\frac{d}{d t}\langle e \vec{E} \cdot \vec{r}\rangle_{\Omega} \\
& \rightarrow \frac{d}{d t}\langle\vec{E} \cdot \vec{r}\rangle_{\Omega}=0
\end{aligned}
$$

Here $W=1 / 2 m\left\langle v^{2}\right\rangle_{\Omega}$ is the particle energy averaged over one RF period. The solutions for $\vec{r}(t)$ given in Eq. 4.6 are the trajectories which satisfy Eq. 4.8; any deviation from those trajectories allows the particle to transfer energy to/from the electric field.

No closed form solution for the $c_{2 n}, \beta$, exists for all $a, q$, but numeric methods can be applied to determine the constants. The application of Eq. 4.3 to Eq. 4.6 gives a recursion relation for the $c_{2 n}$ and $\beta$ that can be solved to arbitrary precision. For our trapping parameters, the $n=0$ coefficient dominates the solution and thus the largest amplitude particle motion occurs at the macromotion frequency,

$$
\omega_{z} \equiv \beta_{z} \frac{\Omega}{2}
$$

Numeric integration of the motion for a particle in our trap is shown in Fig. 4.3 with $V_{D C}=0, V_{A C}=540 V_{p k}$, and $\Omega=8.5 \mathrm{MHz}$. The macromotion frequency for these parameters is $540 \mathrm{kHz}$. The next largest motion happens at the micromotion frequencies, $\omega_{z}=\Omega(1 \pm \beta)$, with amplitude $\sim 10 \%$ of the macromotion. Fig. 4.4 shows the frequency and amplitude of the analytic solutions for the motion of an 
ideal trapped particle (solid line) with the drive frequency superimposed (dashed line).

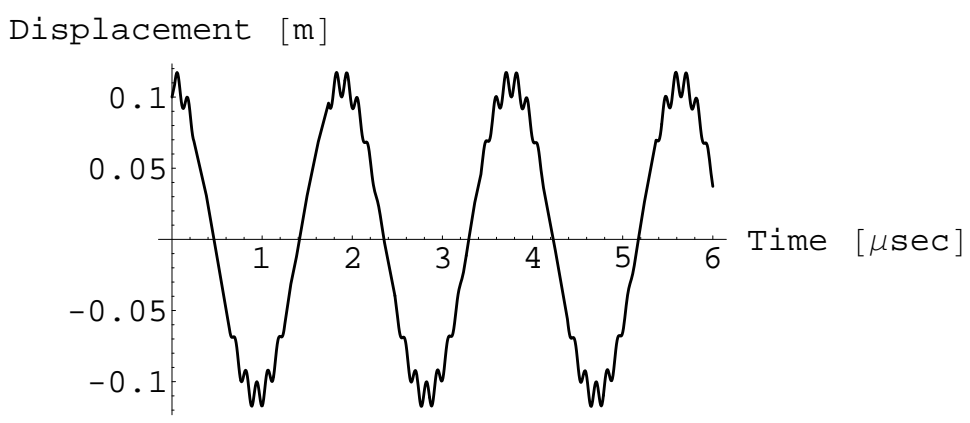

Figure 4.3: One dimensional plot of ion motion in an ideal potential for $V_{D C}=$ $0, V_{A C}=540 V_{p k}$. Note the large amplitude macromotion with smaller micromotion.

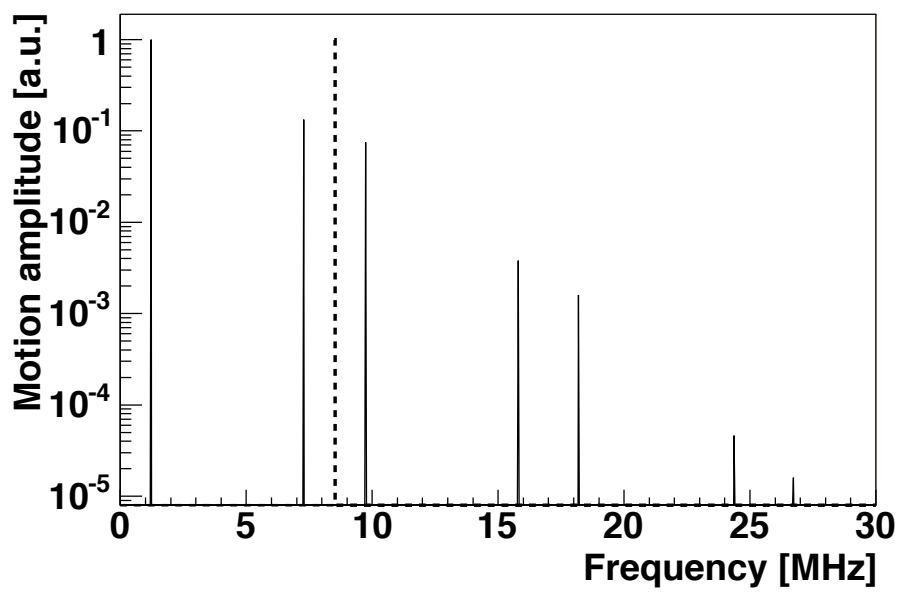

Figure 4.4: Fourier transform of the trapped ion motion (solid line). The drive frequency is $8.5 \mathrm{MHz}$ (dashed line). Note that there is no component of the ion motion at the drive frequency. 


\subsection{The adiabatic approximation}

An alternate treatment of ion trapping called the adiabatic approximation was developed by Dehmelt in 1967 using the effective potential formalism found in Landau $[56,57]$. Consider an ion moving in an inhomogeneous electric field, $E(z, t)$, with a $\mathrm{DC}$ component, $E_{0}(z)$, and $\mathrm{AC}$ term, $E_{\Omega}(z) \cos \Omega t$. Assume the particle trajectory can be described by a small, high frequency micromotion at the drive frequency, $\zeta(t)$, superimposed upon a large, slow macromotion, $\bar{z}$, such that

$$
\begin{aligned}
z(t) & =\bar{z}+\zeta(t) \quad \text { and } \\
\partial \zeta / \partial t & \gg \partial \bar{z} / \partial t .
\end{aligned}
$$

Expanding the electric field around $\bar{z}$ to first order in $\zeta$, the equation of motion is

$$
\frac{d^{2} \bar{z}}{d t^{2}}+\frac{d^{2} \zeta}{d t^{2}}=-\frac{e}{m}\left[E_{0}(\bar{z})+E_{\Omega}(\bar{z}) \cos \Omega t+\zeta \frac{d}{d z} E(\bar{z})\right] .
$$

Collecting together the terms with high frequency components gives an equation for $\zeta(t):$

$$
\frac{d^{2} \zeta}{d t^{2}}=-\frac{e}{m}\left[E_{\Omega}(\bar{z}) \cos \Omega t+\zeta \frac{d}{d z} E(\bar{z})\right] .
$$

The fundamental assumption of the adiabatic approximation is that the high frequency motion of the particle is small compared to the spatial inhomogeneities in the electric field. In other words, the second term on the right hand side of Eq. 4.13 is small compared to the first term and can be neglected, giving

$$
\begin{aligned}
\frac{d^{2} \zeta}{d t^{2}} & =-\frac{e}{m} E_{\Omega}(\bar{z}) \cos \Omega t \\
\zeta & =\frac{e}{m \Omega^{2}} E_{\Omega}(\bar{z}) \cos \Omega t
\end{aligned}
$$

The micromotion is 180 degrees out of phase from the driving force with an amplitude proportional to the distance from the trap center. We solve for $\bar{z}$ by substituting 
Eq. 4.14 into Eq. 4.12,

$$
\frac{d^{2} \bar{z}}{d t^{2}}=-\frac{e}{m}\left[E_{0}(\bar{z})+\frac{e}{m \Omega^{2}} E_{\Omega}(\bar{z}) \cos \Omega t \frac{d}{d z}\left(E_{0}+E_{\Omega} \cos \Omega t\right)\right] .
$$

We are only interested in the slow behavior of $\bar{z}$, so we average over one period of $\Omega$, leaving

$$
\begin{aligned}
\frac{d^{2} \bar{z}}{d t^{2}} & =-\frac{e}{m} \frac{\Omega}{2 \pi} \int_{0}^{2 \pi / \Omega} d t\left[E_{0}(\bar{z})+\frac{e}{m \Omega^{2}} E_{\Omega}(\bar{z}) \cos \Omega t \frac{d}{d z}\left(E_{0}+E_{\Omega} \cos \Omega t\right)\right] \\
& =-\frac{e}{m} E_{0}(\bar{z})+\frac{e^{2}}{m^{2} \Omega^{2}} \frac{\Omega}{2 \pi} \int_{0}^{2 \pi / \Omega} d t\left[E_{\Omega}(\bar{z}) \cos ^{2} \Omega t \frac{d E_{\Omega}}{d z}\right] \\
& =-\frac{e}{m} E_{0}(\bar{z})+\frac{e^{2}}{2 m^{2} \Omega^{2}} E_{\Omega}(\bar{z}) \frac{d E_{\Omega}}{d z} \\
& =-\frac{e}{m} E_{0}(\bar{z})+\frac{e^{2}}{4 m^{2} \Omega^{2}} \frac{d E_{\Omega}^{2}}{d z} .
\end{aligned}
$$

Eq. 4.16 suggests a more intuitive description of the macromotion in terms of the pseudo-potential, $\psi(\bar{z})$,

$$
\begin{aligned}
& \bar{F}(\bar{z})=-e \nabla \psi(\bar{z}), \text { where } \\
& \psi(\bar{z}) \equiv \psi_{0}+\frac{e}{4 m \Omega^{2}} E_{\Omega}^{2}(\bar{z}) .
\end{aligned}
$$

This result is easily generalized to three dimensions. The pseudo-potential formulation is particularly attractive because it allows the ion trap dynamics to be modeled as a static harmonic potential with an absolute minimum, ignoring the effects of the high frequency driving field.

Two immediate applications of the pseudo-potential formulation become obvious from inspection of Eq. 4.17. First, the axial pseudo-potential depth, $\bar{D}_{z}$, can be determined given the form of the electric field. For the quadratic potential of our ion 
trap at zero DC field, the three-dimensional pseudo-potential and trap depth are

$$
\begin{aligned}
\psi(\bar{r}, \bar{z}) & =\bar{D}\left[\frac{\bar{r}^{2}+4 \bar{z}^{2}}{4 z_{0}^{2}}\right], \\
\bar{D}_{z} & \equiv \frac{e V_{A C}^{2}}{m \Omega^{2} z_{0}^{2}}=\frac{q_{z}}{4} V_{A C} .
\end{aligned}
$$

Second, the frequency of the axial macromotion is determined by recognizing that the pseudo-potential in Eq. 4.18 generates a harmonic restoring force with axial "spring constant" $k_{z}=2 e \bar{D}_{z} / z_{0}^{2}$. By analogy with the mechanical harmonic oscillator, the macromotion frequency is

$$
\begin{aligned}
\bar{\omega}_{z} & =\sqrt{2 \frac{e^{2} V_{A C}^{2}}{m^{2} \Omega^{2} z_{0}^{4}}} \\
& =\sqrt{2} \frac{e V_{A C}}{m \Omega z_{0}^{2}} \\
& =\frac{q_{z}}{2 \sqrt{2}} \Omega
\end{aligned}
$$

For small $q_{z}$, this reproduces the result of Eq. 4.9.

The operating parameters and resultant frequencies of motion for the hyperbolic trap used in these experiments are tabulated in Table 4.1. Note that the calculated trap depth is $\bar{D}_{z}>15.4 \mathrm{eV} \sim 180,000 \mathrm{~K}$ and that the macromotion frequency calculated numerically and calculated using the adiabatic approximation agree. Finally, when the assumptions in Eq. 4.11 are violated, the approximation and the resulting intuition must be discarded in favor of results from the full equations of motion.

\subsection{Non-ideal traps}

The ideal quadratic potential assumed in the previous section does not exist in the laboratory. A partial list of deviations from the ideal includes DC contact voltages on the trap electrodes, mechanical truncation of the electrodes, gravity, electrode imperfections and mis-alignment, ambient magnetic fields, collisions with residual gas molecules, and if multiple ions are trapped, coulomb interactions among trapped 


\begin{tabular}{lcc}
\hline Parameter & Low Voltage & High Voltage \\
\hline \hline Ring diameter & $5 \mathrm{~mm}$ & $5 \mathrm{~mm}$ \\
Axial spacing $\left(z_{0}\right)$ & $2 \times 1.77 \mathrm{~mm}$ & $2 \times 1.77 \mathrm{~mm}$ \\
Frequency $(\Omega)$ & $8.5 \mathrm{MHz}$ & $8.5 \mathrm{MHz}$ \\
RF voltage $\left(V_{p k}\right)$ & 440 & 700 \\
$q_{z}=-2 q_{r}$ & .14 & .23 \\
Approx. macro. freq. $\left(\omega_{z}=2 \omega_{r}\right)$ & $421 \mathrm{kHz}$ & $691 \mathrm{kHz}$ \\
Numeric macro. freq. $\left(\omega_{z}=2 \omega_{r}\right)$ & $433 \mathrm{kHz}$ & $695 \mathrm{kHz}$ \\
Axial depth $\left(\bar{D}_{z}\right)$ & $15.4 \mathrm{eV}$ & $40.3 \mathrm{eV}$
\end{tabular}

Table 4.1: Parameters used with $5 \mathrm{~mm}$ hyperbolic trap. The approximate macromotion frequency is calculated using the adiabatic approximation while the numeric solution is found via the Fourier transform of the numerically integrated equations of motion.

ions. Collisions will be covered in detail in the following chapter, this section will address the other imperfections.

In the context of single ion tagging for EXO, deviations from ideal trapping only concern us when they prevent identification of trapped ions. Trapped ions' fluorescence cannot be seen for several reasons: a) the signal is reduced by random motions so that it cannot be distinguished from background or b) the ions exit the trap. Consequently, in this section we will explore the effect of electrode imperfections on the temperature and stability of trapped ions.

Static forces applied to the trapping region cannot unload ions from the trap as long as their magnitude is small compared to the applied AC trapping fields and the stability requirements of Fig. 4.2 are satisfied. Assuming the perturbations are small and the adiabatic assumption of Eq. 4.11 holds, static DC fields modify the pseudo-potential $\psi(\bar{r}, \bar{z})$,

$$
\psi^{\prime}(\bar{r}, \bar{z})=\psi_{0}(\bar{r}, \bar{z})+\psi_{1}(\bar{r}, \bar{z})
$$

where $\psi_{0}$ is the unperturbed pseudo-potential from Eq. 4.18 and $\psi_{1}$ is the non-ideal 
contribution. Consider an expansion of $\psi_{1}$ around the origin,

$$
\psi_{1}=\psi_{1}(0,0)+\partial_{r} \psi_{1} r+\partial_{z} \psi_{1} z+\mathcal{O}\left(r^{2}, z^{2}\right)
$$

and assume that the contributions of second order and higher can be neglected. Both gravity and contact potentials fit this prescription since the gravitational potential is linear and the contact potentials, mainly formed via barium plating on one side of the trap, create dipole fields. Now $\psi^{\prime}$ takes the form

$$
\psi^{\prime}(\bar{r}, \bar{z})=\bar{D}\left[\frac{\bar{r}^{2}+4 \bar{z}^{2}}{4 z_{0}^{2}}\right]+\psi_{1}(0,0)+\partial_{r} \psi_{1} r+\partial_{z} \psi_{1} z .
$$

After completing the square and neglecting the constant term, the potential can be expressed as

$$
\begin{aligned}
\psi^{\prime}(\bar{r}, \bar{z}) & =\bar{D}\left[\frac{\left(\bar{r}-r_{c}\right)^{2}+4\left(\bar{z}-z_{c}\right)^{2}}{4 z_{0}^{2}}\right], \quad \text { with } \\
r_{c} & =\frac{2 z_{0}^{2}}{\bar{D}} \partial_{r} \psi_{1} \quad \text { and } \\
z_{c} & =\frac{z_{0}^{2}}{2 \bar{D}} \partial_{z} \psi_{1} .
\end{aligned}
$$

The effect of the linear perturbation is to move the trap center from $(0,0)$ to $\left(r_{c}, z_{c}\right)$. Similarly, quadratic perturbations affect the potential depth, and higher order terms lead to an-harmonic terms with (assumed) small effect near the trap center. At large radius, the an-harmonic terms can lead to unloading. Shifting the trap center from $r=z=0$ increases the magnitude of the micromotion, but does not change the shape of the pseudo-potential. The increased micromotion will frequency modulate the absorption spectrum, causing a reduction of the fluorescence intensity. Except in extreme cases, the increased micromotion does not render the ions invisible. For experiments which depend on minimal micro-motion, the external potentials can be canceled using an appropriate set of bias electrodes, returning the ion to the trap center and minimizing the micromotion [58].

The effects of misalignment or asymmetries in the trapping electrodes present a 
more serious problem since the applied RF trapping voltage is large by definition. Moreover, the perturbations now occur at the oscillating frequency so the adiabatic approximation is no longer correct and the full equations of motion must be solved. A non-ideal trapping potential can be expanded in terms of the Legendre polynomials,

$$
\Phi(r, \theta)=\left(V_{D C}+V_{A C} \cos \Omega t\right) \sum_{n=2}^{\infty} c_{n}\left(\frac{r}{d}\right)^{n} P_{n}(\cos \theta)
$$

and numerically solved for the trapped particle's motion [59]. Here, $r$ is the radius from the center of the trap, $z=r \cos \theta$, and $d$ is the diameter of the ring electrode. Eq. 4.25 implicitly assumes cylindrical symmetry for which $m=0$ and the coefficients for odd values of $n$ are zero. The quadrupole term with $n=2$ is the ideal trapping potential, the higher order terms with $n=4,6,8 \ldots$ are treated as perturbations. The Mathieu equation of Eq. 4.3 is modified to

$$
\frac{d^{2} u_{i}}{d \tau^{2}}+\left(a_{i}-2 q_{i} \cos 2 \tau\right) u_{i}=-\left(a_{i}-2 q_{i} \cos 2 \tau\right) \frac{\partial \bar{\phi}}{\partial u},
$$

where $\bar{\phi}$ is the perturbing potential. The inhomogeneous term on the right side of the equation couples the frequencies of motion to the RF frequency, heating the particle. In particular, when

$$
n_{r} \omega_{r}+n_{z} \omega_{z}=k \Omega
$$

with $n_{r}, n_{z}, k$ integer, the harmonics of the particle motion coincide with harmonics of the drive frequency and energy is efficiently coupled into the particle's motion [60, 61]. When there are significant higher order contributions to the potential expansion, the $a, q$ stability region develops "black holes" where the macromotion frequencies satisfy Eq. 4.27 and resonate with harmonics of the driving field, heating the ions out of the trap. These instabilities have been observed in several experiments; see for instance Refs. [62]. Fortunately, the instabilities for lowest $n_{z}, n_{r}$, (which arise from the lowest order multipole imperfections) occur at high $q$, and their contributions can be minimized by running the trap at low $q_{z}$. Nonetheless, the high order harmonic resonances can heat trapped ions even when they do not cause the ions to leave the 
trap.

Empirically, in the absence of any particular cooling mechanism, trapped ion clouds reach a "temperature" equal to about $1 / 10$ th the trap depth $\bar{D}[56,63,64]$. The combined contributions of the static non-quadratic potentials, the imperfections in the trapping fields, and coulomb interactions among the particles raises the temperature of the trapped ions. Although these effects reduce the fluorescence signal to noise ratio, ions remain stably trapped and the signal can be integrated for arbitrary lengths of time in order to positively tag single barium ions. 


\section{Chapter 5}

\section{Trapping with buffer gas}

Ion traps have been used with buffer gases since their inception. The first demonstrations of three-dimensional electrodynamic confinement used trace amounts of air to cool the otherwise violent motion of trapped particles [65, 54]. The addition of air damped the particle velocity and allowed them to crystallize into a regular pattern. Today, RF ion traps used for mass spectroscopy frequently use helium to cool trapped ions and improve the mass selectivity [66].

Both of these examples, and most applications in the literature, use a background gas with mass much less than that of the trapped particle. The reasons for this are two-fold. First, for the trapped gas droplets and aluminum particles used by Straubel and Wuerker, every gas atom weighs less than the trapped particle. Similarly, most interesting molecules for mass spectroscopy (DNA, proteins) weigh hundreds and thousands of grams per mole, much more than the standard buffer gases. Second, light buffer gases perturb ion trap dynamics the least and only modify the energy distribution of trapped ion clouds. This fact is a consequence of momentum conservation and will be discussed in $§ 5.4 .1$.

In this chapter, we develop a model for the effect of collisions between trapped barium and noble gas buffers. To begin, we critically review the available literature on the subject. Then we develop a theory of collisions between ions and non-polar buffer gases. Finally, we consider the effect of collisions on the trapped ion spatial distribution, energy distribution, and trapping lifetime. In particular, we examine 
the effect of buffer gas mass on single ion visibility.

\subsection{Collisions in the literature}

Most discussions about collisions in ion traps refer to Dehmelt's arguments in Ref. [56]; we do the same. Dehmelt works within the adiabatic approximation (see §4.2) and begins by considering ion clouds colliding with fixed, randomizing scatterers. Physically, this is equivalent to hard sphere collisions (randomizing) with an infinitely massive (fixed) buffer gas. The center of mass of a collection of ions, each of which collides with a fixed scatterer near $\bar{z}$, is stationary because the velocities are randomized. The stationary ensemble takes energy from the electric field, accelerating the center of mass with $\ddot{z}=-e / m \nabla V(\bar{z})$. When averaged over the macro- and micromotions, this acceleration yields an average energy gain equal to the energy of the ensemble at the time of the collision. Dehmelt parameterizes the heating as

$$
\frac{\partial 3 k \mathbf{T}_{i}}{\partial t}=a\left(m_{I} / m_{B G}\right) 3 k \mathbf{T}_{i} / t_{r}
$$

where, $k$ is Boltzmann's constant, $\mathbf{T}_{i}$ is the ion cloud temperature and $t_{r}$ is the time in between collisions. Note that the ion cloud temperature is only well-defined within the adiabatic approximation. The factor, $a\left(m_{I} / m_{B G}\right)$, depends on the mass of the trapped ion, $m_{I}$, and the mass of the scatterer, $m_{B G}$. By the above argument, $a=+1$ when $m_{B G}>m_{I}$. On the other hand, when the ions collide with much lighter, cold partners, momentum conservation ensures that an elastic collision barely perturbs the ion's motion and the ion is not accelerated by the electric field. Nonetheless, the cold, light collision partner carries away energy, leading to a damping of the ion temperature and hence $a\left(m_{I} / m_{B G}\right)$ is negative when $m_{B G} \ll m_{I}$.

Dehmelt briefly considers collisions with equal mass scatterers, noting that the most extreme case is a head-on collision of an ion with average energy, $W$, with a stationary buffer gas atom of equal mass. The collision stops the ion's motion and leaves it with no kinetic energy; less-direct collisions cause a smaller change in energy. After a head on collision, the stationary ion gains energy from the RF field equal to, 
on average, $W$; in other words, an ion does not change energy via collisions with equal mass scatterers. This statement is then taken to imply, without proof, that for all collisions with an equal mass buffer gas

$$
|a(1)| \ll 1 .
$$

Having concluded that equal mass scattering does not change the energy distribution of trapped ions, Dehmelt then continues by noting that the most likely equal mass scatterers are the neutral parent gas atoms for which charge exchange reactions dominate. The charge exchange reaction results in no net change of the ion energy or ion number.

Applied to barium tagging in xenon gas, Dehmelt's theory of collisions for similar mass particles is incomplete for three reasons. First, the spatial distribution and the unloading rate are not directly considered. According to Eqs. 5.1 and 5.2, barium ions colliding with xenon will not gain energy on average. However, even without increasing the ion temperature, collisions create diffuse ion clouds such that: a) the low ion density in the laser illuminated volume decreases the ion fluorescence, and b) the spatial distribution extends beyond the finite trap dimensions and ions unload on contact with the trap electrodes. More critically, Dehmelt's argument relies on the validity of the adiabatic approximation. In the presence of collisions with massive atoms, the particle motion can no longer be decomposed into the macromotion, $\bar{z}$, and the high frequency micromotion, $\zeta$. The adiabatic constraint, $\zeta \partial E(\bar{z}) / \partial z \ll 1$, no longer applies and the electric field can do work on the ion. Even the relatively slow macromotion velocity, when scattered against (or with) the electric field can lead to a substantial change in the ion energy. Finally, Dehmelt only considers the time-averaged, macroscopic ion cloud temperature while neglecting the fluctuations in the individual ion orbits. Even when there is no net energy gain, low-probability sequences of energy-gaining collisions can lead to trap unloading.

One way to address the effect of collisions is to study the damped Mathieu equation. For instance, Hasegawa et al. treat the simplest possible case by adding a linear 
velocity damping term to Eq. 4.3,

$$
\frac{d^{2} u_{i}}{d \tau^{2}}+\gamma \frac{d u}{d \tau}+\left(a_{i}-2 q_{i} \cos 2 \tau\right) u_{i}=0
$$

where $\gamma$ is the rate-constant of the exponential velocity damping [67]. In their words, "this situation is appropriate particularly for collisional cooling of a microparticle by a background gas." The damping term greatly enlarges the stability region, in accordance with the intuitive expectation that damping lessens the high-amplitude motion which leads to trap unloading. Somewhat counter-intuitively, the damping also shifts the stability region, causing instabilities for previously stable values of $a$, and $q$. Like the frequency of a damped harmonic oscillator in classical mechanics (c.f. José and Saletan $§ 3.3[68]$ ), the macromotion frequency of a trapped particle is shifted by the damping term. Hasegawa et al. find the calculated shift to be in excellent agreement with the data of Wuerker et al. for trapped aluminum particles. Nasse et al. [69] report experimental measurements of trapped glycerin droplets at atmospheric pressures and also find quantitative agreement with the prediction from $[67]$.

Many authors have published studies of ion clouds cooled by helium; here we present a representative sample. Lunney et al. report experimental measurements of the cooling of large clouds of potassium ions (20,000 ions) by $\sim 1$ mtorr of helium buffer gas. The buffer gas cools the cloud temperature from $\sim 6 \mathrm{eV}$ to $0.3 \mathrm{eV}$, and compresses the cloud spatially [70]. Cutler et al. have cooled mercury ion clouds of one million atoms to room temperature using $1 \times 10^{-5}$ torr of helium gas, extending the storage time and increasing the ion cloud density [63]. Other authors obtain similar results [71, 72]. Although these results are encouraging, the buffer is much lighter than the ions and do not apply to the case of Ba-II in Xe buffer.

Finally, Ref. [73] presents experimental evidence of ion unloading by collisions with a heavy buffer gas. Their experiment studies the unloading rate of trapped magnesium ions (24 AU) colliding with the much heavier krypton buffer gas (84 AU). Again, the work is not directly applicable to our situation, mainly because the trapped ion is substantially lighter than the buffer gas. However, the paper helps provide the 
key insight upon which this work is based and so deserves some discussion. In their theory, the rate of loss, $\gamma_{\text {loss }}$, is given by the probability that a scattering event raises the particle energy above the trap depth, $D$. This can be expressed as a function of the trapped ion energy and the collision probability by

$$
\gamma_{\text {loss }}=\int d v^{3} f(v, T) \times \int_{E^{\prime}>D} P\left(E \rightarrow E^{\prime}\right) d E^{\prime},
$$

where $f(v, T)$ is the ion velocity distribution at temperature, $T$, and $P\left(E \rightarrow E^{\prime}\right)$ is the probability that a collision raises the ion energy from $E$ to $E^{\prime} . P\left(E \rightarrow E^{\prime}\right)$ is evaluated numerically for the particular trap parameters and all possible collisions, leading to the conclusion that, on average, ten $\mathrm{Mg}$ - Kr collisions are required to unload an ion. The experiment also shows that the addition of substantial pressures of helium as a buffer gas can slow the $\mathrm{Mg}$ - Kr unloading but cannot eliminate it altogether. Like Dehmelt's discussion, the authors are mainly considered with large ion clouds, and do not consider single particle dynamics. However, the authors demonstrate that a series of single ion-gas collision can lead to unloading, a key premise for this thesis.

\subsection{Ion-ion collisions}

Long range coulombic interactions among ions present a particularly thorny problem. Quite complicated and beautiful behaviours arise for multiple trapped ions. For instance, Hoffnagle et al. find that the behaviour of two trapped ions can only be explained using chaos theory [74]. Blümel et al. characterize the multi-ion operating regime in great detail, finding a pure chaotic regime, a Mathieu regime, and a quasiperiodic regime [51, 75].

In principle, we can trivially eliminate coulomb interactions by operating the trap with only a single ion. Conceptually, all of the analysis in sections $\S 5.3-5.5$ applies to a single ion, and ion-ion interactions don't apply. In practice, however, we maximize our signal to noise ratio by loading many ions into the trap at once and studying their collective behaviour. We load 1 to 1,000 ions, depending on the situation. For the purposes of this thesis, we must ensure that ion trap dynamics are dominated by 
ion-neutral collisions and that ion-ion collisions, though never entirely absent, play a secondary role.

To this end, we calculate the density of $N_{I}$ ions at a temperature, $T$, and show that the density is small compared to the background gas density. In order to calculate the volume occupied by the ions, we compare the pseudo-potential energy to the thermal energy. Ions fill the trap until the pseudo-potential energy, $\psi(\bar{r}, \bar{z})$, is equal to the temperature of the ion cloud, such that

$$
e \psi\left(\bar{r}_{T}, 0\right)=e \psi\left(0, \bar{z}_{T}\right)=3 k T
$$

with the thermal cloud axes, $\bar{r}_{T}$ and $\bar{z}_{T}$, defining the maximum extent of the ion cloud along the radial and axial direction. Using Eq. 4.18, the thermal cloud extends to

$$
\bar{z}_{T}^{2}=2 \bar{r}_{T}^{2}=\frac{3 k T}{e \bar{D}} z_{0}^{2}
$$

and the corresponding volume is

$$
V_{T}=4 / 3 \pi \bar{z}_{T} \bar{r}_{T}^{2}=4 / 3 \pi\left(\frac{3 k T z_{0}^{2}}{e \bar{D}}\right)^{3 / 2}
$$

Table 5.2 tabulates the thermal volumes for the two trap configurations discussed previously and used in this work. We have trapped at most 1,000 ions at ion densities of $\rho=1000 / V_{T}=1$ to $5 \times 10^{8}$ ions $/ \mathrm{cm}^{3}$. Note that this density is only well defined when the ion cloud has a temperature; this description does not apply to single ions in UHV, but rather to ion clouds in equilibrium. These extremely low densities are equivalent to gas pressures of $10^{-12}$ torr. Charged plasma calculations are notoriously difficult because of the infinite coulomb cross section and we do not calculate them here [76]. However, since the buffer gas densities are between 6 and 10 orders of magnitude higher than the trapped ion density, we choose to neglect ion-ion collisions compared to ion-neutral collisions for the remainder of this work. 


\begin{tabular}{lcc}
\hline Parameter & Low Voltage & High Voltage \\
\hline \hline Axial spacing $\left(z_{0}\right)$ & $2 \times 1.77 \mathrm{~mm}$ & $2 \times 1.77 \mathrm{~mm}$ \\
Axial depth $\left(\bar{D}_{z}\right)$ & $15.4 \mathrm{eV}$ & $40.3 \mathrm{eV}$ \\
Thermal Volume $\left(V_{T}\right)$ & $8.2 \times 10^{-6} \mathrm{~cm}^{3}$ & $1.9 \times 10^{-6} \mathrm{~cm}^{3}$
\end{tabular}

Table 5.1: The volume of the ion cloud in the hyperbolic trap under two operating conditions

\subsection{Brownian motion}

It is appealing to think of a trapped ion in buffer gas as a particular case of a harmonic oscillator undergoing Brownian motion. Using the formalisms developed for other stochastic processes, one might hope to average over many microscopic interactions and make quantitative predictions about the macroscopic behaviour of the trapped ion/buffer gas system. The work of Blatt et al. takes this approach by considering a trapped ion in buffer gas as a parametric oscillator undergoing Brownian motion [77]. To begin, the Mathieu equation (Eq. 4.3) is cast into a Langevin form,

$$
\frac{d^{2} u}{d t^{2}}+\gamma \frac{d u}{d t}+\frac{1}{4} \Omega^{2}(a-2 q \cos \Omega t) u=F(t) / m,
$$

where $F(t)$ is a Gaussian random force with $\left\langle F(t) F\left(t^{\prime}\right)\right\rangle=2 D \delta\left(t-t^{\prime}\right) m^{2}$. The damping constant, $\gamma$, and the diffusion constant, $D$, encapsulate all of the physics involved in collisions. Note that Eq. 5.8 employs a slightly different convention than Eq. 4.3 in which the time, $t$, is not scaled by $\Omega / 2$ and the $1 / 4 \Omega^{2}$ must be included. This form of the Langevin equation derives from Brownian motion, and includes the implicit assumption that the ion is much heavier than the buffer gas $[78,79]$. The treatment fails with a heavy background gas since massive scattering cannot be described by a slow velocity damping and a fast Gaussian force; single impacts cause large changes in the velocity. Furthermore, in order to maintain equations with an analytic solution the force $F(t)$ overly simplifies the interactions by ignoring the spatial and velocity components of the collisions.

Within the context of Brownian motion, however, Eq. 5.8 leads to the very nice 
result,

$$
\overline{\left\langle v^{2}\right\rangle}=\left|1+\frac{\frac{1}{8} \Omega^{2} q^{2}}{\omega^{2}}\right|\left(\frac{D}{\gamma}\right)
$$

where $\omega$ is the macromotion frequency and $\overline{\left\langle v^{2}\right\rangle}$ is the mean square velocity of the ion's Gaussian velocity distribution. For a harmonic oscillator subject to Brownian forces, $\overline{\left\langle v^{2}\right\rangle}=D / \gamma$, the first term in Eq. 5.9 [78]. If the adiabatic approximation was valid and the pseudo-potential was merely a static harmonic potential, this would be the only contribution to $\overline{\left\langle v^{2}\right\rangle}$. The second term, proportional to $1 / 8 \Omega^{2} q^{2} / \omega^{2}$, is identified with RF heating and highlights the perils of neglecting the RF field when calculating the effect of collisions. The Gaussian spatial distribution has the mean square displacement, $\overline{\left\langle x^{2}\right\rangle}$, proportional to the mean square velocity, ie. $\overline{\left\langle x^{2}\right\rangle} \propto \overline{\left\langle v^{2}\right\rangle}$, where the exact ratio is determined by $a, q$, and $\Omega$.

Like many treatments of stochastic systems, Blatt et al. make no attempt to calculate $D$ and $\gamma$ from the microscopic interaction. In fact, the discussion aims for a general application to macroscopic observables, independent of the microscopic source of the gaussian force, $F(t)$. Instead of specifying particular interactions, they assert that physical parameters lead to $\gamma / \Omega \simeq 10^{-7}-10^{-4}$ and leave $D$ undefined. Although it may be possible to calculate $\gamma$ and $D$ for Ba-II in Xe from knowledge of the microscopic behaviour and thus derive the macroscopic behaviour of the system, we were unable to do so for this work. Instead, we choose to calculate trapped ion dynamics using numeric simulations.

\subsection{Ion-neutral collisions}

We have shown the available analytic techniques to be incapable of calculating the spatial distribution for trapped ions in heavy buffer gases, but we have yet to develop a theory with which we can interpret our data. Instead of using analytic calculations, we use numeric Monte Carlo methods to simulate the trapped dynamics of a single trapped ion. We require two additional calculations in order to realistically simulate the motion of a trapped ion; the first piece is elastic collision kinematics, and the second is a realistic description of the ion-neutral collisions. 


\subsubsection{Scattering kinematics}

The maximum velocity change imparted by a collision depends on the mass of the scattering particle. Consider the change in velocity, $\Delta \vec{v}_{I}$, of a trapped ion with velocity, $\vec{v}_{I}$, and mass, $m_{I}$, scattering off of a background particle of $\vec{v}_{B G}, m_{B G}$. The center of mass for this system moves with velocity

$$
\vec{V}_{C O M}=\frac{m_{I} \vec{v}_{I}+m_{B G} \vec{v}_{B G}}{m_{I}+m_{B G}} .
$$

In the Center of Mass frame $(\mathrm{CM})$, the velocity vector $\vec{v}_{I}$ is transformed to $\vec{u}_{I}=$ $\vec{v}_{I}-\vec{V}_{C O M}$. Momentum and energy conservation ensure that the magnitude of the $\mathrm{CM}$ vector before and after an elastic collision are equal: $\left|u_{I}\right|=\left|u_{I}^{\prime}\right|$. In the CM frame, the particle scatters with polar angle, $\theta$, and azimuthal angle, $\phi$. Since the collisions are azimuthally symmetric, we neglect $\phi$ in the following. With the rotation matrix, $R(\theta), \vec{u}_{I}^{\prime}=R(\theta) \vec{u}_{I}$, we calculate the collision-induced velocity change in the laboratory frame,

$$
\Delta \vec{v}_{I}=\frac{(R(\theta)-1)}{1+m_{I} / m_{B G}}\left(\vec{v}_{I}-\vec{v}_{B G}\right) .
$$

Eq. 5.11 explains why light buffer gases are used for cooling. For hot ions (a few $\mathrm{eV}$ ), the velocity of the room temperature buffer gas atoms may be neglected compared to the ion's velocity. Then $\Delta \vec{v}_{I} / \vec{v}_{I} \propto 1 /\left(1+m_{I} / m_{B G}\right)$. For barium ions in helium buffer gas, $1 /\left(1+m_{I} / m_{B G}\right)=0.0286$. Obviously, collisions with helium do not cause large perturbations in the ion orbit. In contrast, for a collision of a barium ion with a cold xenon atom, $1 /\left(1+m_{I} / m_{B G}\right)=0.5$.

To calculate the buffer gas cooling efficiency, we consider the change in energy of the ion. Our assumption that the ion is much hotter than the buffer gas also implies that the velocity of the buffer gas atom before the collision is negligible compared to 
its final velocity. In this case, the maximum change in energy is given by

$$
\begin{aligned}
\Delta E_{I}=-\Delta E_{B G} & =\frac{1}{2} m_{B G}\left(v_{B G}+\Delta v_{B G}\right)^{2}=\frac{1}{2} m_{B G} \Delta v_{B G}^{2} \\
& \propto \frac{1}{2} m_{B G}\left(\frac{2}{1+m_{B G} / m_{I}} v_{I}\right)^{2} \\
& \propto 4 \frac{m_{B G} / m_{I}}{\left(1+m_{B G} / m_{I}\right)^{2}} E_{I} .
\end{aligned}
$$

The fraction $\left(m_{B G} / m_{I}\right) /\left(1+m_{B G} / m_{I}\right)^{2}$ has a maximum when $m_{B G}=m_{I}$, however an equal mass buffer also implies large orbital perturbations. Eq. 5.12, multiplied by the collision frequency, gives an upper limit for the buffer gas cooling rate. Helium is the most used buffer gas because it does not distort the trapping dynamics while still damping the trapped particles' energy.

\subsubsection{Induced dipole interactions}

In order to model the dynamics of trapped ions in buffer gas, we must also understand the microscopic behaviour of the ion-buffer gas collisions. At large distances, the monopole-induced dipole is the strongest interaction between an ion and non-polar gas atom. The interaction potential is

$$
V_{D}(\vec{r})=-\frac{\alpha}{2} \frac{e^{2}}{r^{4}}
$$

where $\alpha$ is the polarizability of the buffer gas and $r$ is the distance between the atom and the ion [76]. For elastic collisions which conserve angular momentum and energy, the radial momentum as a function of radius is

$$
p_{r}(r)=m \frac{d r}{d t}= \pm p_{0}\left[1-\left(\frac{b}{r}\right)^{2}-\frac{V(r)}{E}\right]^{1 / 2},
$$

with the initial momentum $p_{0}$, the mass $m$ and the impact parameter $b$ [80]. We work in a frame with the neutral gas atom stationary at the origin and the ion moving with 
velocity $v$. The deflection angle for the ion is,

$$
\Theta=\pi-\int_{-\infty}^{\infty} \frac{v b}{r^{2}} d t
$$

At the point of closest approach, $r_{0}$, the ion is moving purely tangentially to the gas atom and has no radial velocity, ie. $p_{r}\left(r_{0}\right)=0$. Using this fact and transforming variables we obtain the scattering angle in terms of $p(r)$,

$$
\Theta(b, E)=\pi-2 \int_{r_{0}}^{\infty} \frac{b / r^{2}}{\sqrt{1-(b / r)^{2}-V(r) / E}} d r .
$$

For the induced dipole potential, we can solve Eq. 5.14 for $p_{r}\left(r_{0}\right)=0$ to find

$$
r_{0}=\sqrt{\frac{b^{2}+\sqrt{b^{4}-4 \frac{\alpha}{2} \frac{e^{2}}{E}}}{2}} .
$$

Using this equation, we define the critical impact parameter,

$$
b_{0}=\left(\frac{2 \alpha e^{2}}{E}\right)^{1 / 4}
$$

for which $r_{0}$ becomes undefined. For collisions with $b \leq b_{0}$, the ion orbits the gas atom several times and the scattering angle is effectively randomized. As an example, barium ions with $1 \mathrm{eV}$ kinetic energy colliding with xenon buffer gas have a critical radius, $b_{0}=3.3 \AA$, while room temperature ions have a critical radius $b_{0}=8.2 \AA$.

Fig. 5.1 shows sample trajectories for a range of impact parameters from $b=b_{0}$ to $b=2 b_{0}$. For the larger impact parameters, the incoming ion is slightly deflected from its initial trajectories; for $b=2 b_{0}$, the ion is deflected by $\Theta=2.1^{\circ}$. As the impact parameter nears the critical value, the scattering angle quickly slews through $\pi$ and the ion enters semi-bound orbits, effectively randomizing the scattering angle. This leads us to define a hard sphere cross-section, first defined by Langevin in the study 


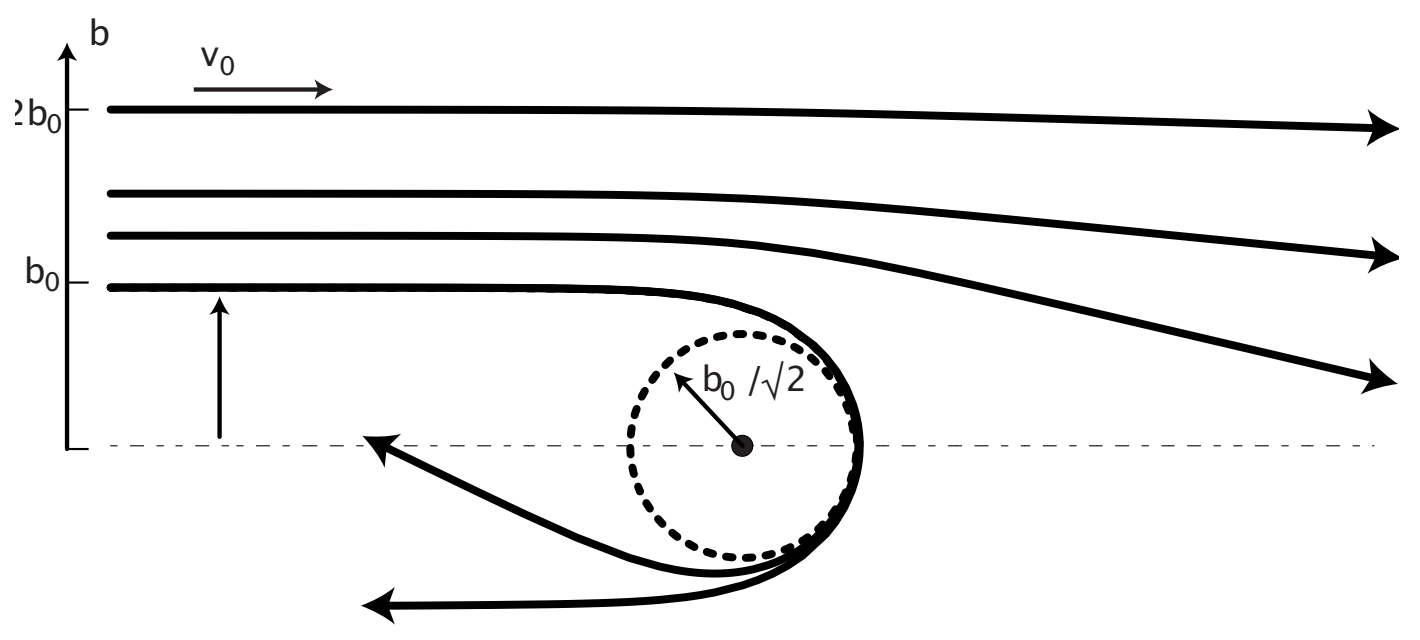

Figure 5.1: Trajectories for ion-induced dipole collisions. The impact parameters range from $b_{0}$ to $2 b_{0}$. Notice that for $b=2 b_{0}$ there is little deflection while for $b=b_{0}$ the ion orbits the fixed center.

of chemical reaction rates [81], as

$$
\sigma_{L}=\pi b_{0}^{2}=\frac{1}{v}\left(\frac{4 \alpha e^{2}}{m}\right)^{1 / 2}
$$

For collisions occurring with this cross-section, the ion orbits the gas atom during the collision, randomizing the scattering angle.

Although $\sigma_{L}$ slightly underestimates the area for which large angle scatterings occur (by $20 \%$ for $\Theta \geq 45^{\circ}$ and by $5 \%$ for $\Theta \geq 90^{\circ}$ ), this cross section has the convenient property that it depends inversely on the particle velocity. Consequently, an ion colliding with a background gas of density, $n_{g}$, has a mean time between collisions of

$$
\tau_{c}=\frac{1}{n_{g} \sigma_{L} v}=\frac{1}{n_{g}}\left(\frac{m}{4 \alpha e^{2}}\right)^{1 / 2}
$$

which is completely independent of the ion velocity. Table 5.2 lists the ion mean free times for the buffer gases used in this work. The highest pressures used in this work are $P \sim 10^{-2}$ torr, corresponding to mean free times of $\tau_{c} \simeq 10^{-5}$ seconds. 


\begin{tabular}{l|cc}
\hline Gas & $\alpha\left[\AA^{2}\right]$ & $\tau_{c} P\left[10^{-7}\right.$ sec torr $]$ \\
\hline Helium & 0.205 & 9.8 \\
Argon & 1.64 & 3.6 \\
Krypton & 2.48 & 2.8 \\
Xenon & 4.04 & 2.2
\end{tabular}

Table 5.2: The mean free time between collisions using the Langevin cross section. The cross section is normalized to the background pressure in torr at room temperature.

\subsection{Scattering in phase space}

An ion trap can easily have a potential depth greater than $10 \mathrm{eV}$, and the temperature of a trapped ion cloud in the absence of cooling is only $10 \%$ of this depth, $\sim 1 \mathrm{eV}$. Even ions in the high energy tail of the Maxwell-Boltzmann distribution lack sufficient energy to overcome the trapping potential. Under these conditions, where can an ion possibly gain the energy required to unload?

The answer lies in the trapped ion's relationship to the driving RF field. Even though the trap depth is $10 \mathrm{eV}$, the driving voltage is $600 \mathrm{~V}$. By changing the phase relationship of the particle's motion to the driving field, the particle can take energy from the RF field and move from a stable orbit to an orbit that intersects an electrode and loses the ion. The following analysis examines the increase in the maximum radius of a trapped ion resulting from collisions.

The duration of a collision is much shorter than any other time scale governing a trapped ion's motion, so a collision causes an instantaneous change in the velocity of the particle while leaving the position constant. For simplicity, we consider onedimensional motion and drop the subscript, $i$, from the parameters $a, q, \beta$ and $c_{2 n}$. Each collision is an independent event, so we are free to set $t=\tau=0$ and, using Eq. 4.6, solve for $A$ and $\phi$ at the time of the collision:

$$
\begin{aligned}
& u(0)=A \cos \phi \sum_{n} c_{2 n} \\
& v(0)=-A \sin \phi \frac{\Omega}{2} \sum_{n} c_{2 n}(2 n+\beta),
\end{aligned}
$$


where $u(0)$ and $v(0)=d u / d t$ are the position and velocity of the ion when the collision occurs. The constant, $A$, defines the maximum radius of the trapped orbit and $\phi$ defines the phase of ion motion with respect to the driving field. $A$ and $\phi$ completely define the orbit; they are a complementary parameter space to $u$ and $v$. In both equations, the summations depend only on $a, q$ and are constant for all time; we denote them $\chi=\sum c_{2 n}$ and $\xi=\sum c_{2 n}(2 n+\beta)$. Note that $\chi$ and $\xi$ are dimensionless and both constants are positive for all $a$ and $q$.

To evaluate the effect of a collision, we take the partial derivatives of Eq. 5.21 and find,

$$
\partial u=\chi[\cos \phi \partial A-A \sin \phi \partial \phi], \quad \partial v=-\frac{\xi \Omega}{2}[\sin \phi \partial A+A \cos \phi \partial \phi] .
$$

During a collision the ion position doesn't change, $\partial u=0$. Solving for the change in maximum radius, $\partial A$, gives

$$
\partial A=-\frac{2 \sin \phi}{\xi \Omega} \partial v
$$

This equation is surprisingly simple; the increase in radius depends only on the change in velocity and the RF phase during a collision. For the $a$ and $q$ used in our experiments, $1 / \xi \geq 4$. From Eq. 5.21, the trapped ion's maximum velocity is

$$
v_{\max }=\frac{A \Omega \xi}{2}
$$

Combining the mass-dependent kinematics of Eq. 5.11 (in one dimension) with Eq. 5.23 gives the maximum orbit increase,

$$
\begin{aligned}
\partial A_{\max } & = \pm \frac{2}{\xi \Omega} \partial v_{\max }= \pm \frac{2}{\xi \Omega} \frac{v_{\max }}{1+m_{I} / m_{B G}} \\
& = \pm \frac{2}{\xi \Omega} \frac{A \Omega \xi}{2} \frac{1}{1+m_{I} / m_{B G}} \\
& = \pm \frac{A}{1+m_{I} / m_{B G}} .
\end{aligned}
$$

Rather than consider the position and velocity of the particle as it orbits the trap center, it is more useful to consider the random walk of the maximum radius, $A$, under 
the impact of a series of collisions. In much the same way that the classic "drunken walk" leads to a Gaussian position distribution, a series of random collisions leads to a Gaussian-like spread in $A$. However, the distribution of $A$ is not a Gaussian because the scattering in Eq. 5.25 is weighted towards large step sizes. In other words, as the ion radius increases, so does the change in the radius. The resulting distribution is not Gaussian but instead a power law.

This formalism highlights the interplay between the trapped ion and the RF field, demonstrating how "low temperature" ion clouds nonetheless unload because of collisions with a massive background gas. Within this context, the notion of the trapped ion's energy and temperature are deprecated in favor of diffusion of the ion through the phase space represented by $A$ and $\phi$.

Although the equations of motion are completely separable, Eq. 5.23 cannot be transformed into the full 3-D treatment merely by replacing the subscript, $i$, to the $A$ and $\phi$. The angular distribution of the collisions couples the three degrees of freedom within the trap. Consequently, an analytic calculation of the spatial distribution using Eq. 5.23 for three dimensions does not seem feasible. Instead, we use Monte Carlo methods to generate realistic space and velocity distributions. 


\section{Chapter 6}

\section{Experimental results}

In this chapter, we present the results of our work trapping barium ions in high pressure xenon buffer gas using an RF Paul trap. After a brief description of the experimental setup, we present the results of single ion trapping studies in ultra-high vacuum. Then we discuss our studies of small ion clouds trapped in the presence of a variety of buffer gases.

\subsection{Basic experimental setup}

A detailed description of the experimental setup can be found in Appendix A; here we briefly describe the setup to provide a context for the following results. As shown schematically in Fig. 6.1, the hyperbolic trap occupies the center of a 4.5 inch, stainless steel, con-flat vacuum cube. Three windows provide optical access for lasers and a long working distance microscope. Several apertures mounted inside the vacuum system block stray laser light from reaching the trap electrodes and reflecting into the collection optics. A tantalum foil, coated with barium from commercial dispenser wire and resistively heated to $\sim 350 \mathrm{C}$, generates a neutral barium vapor. Electrons from a $500 \mathrm{~V}, 100$ microamp electron gun ionize the barium vapor in the trap center, creating Ba-II and Ba-III ions in the trapping region. A 20 l/s Starcell ion pump evacuates the chamber to Ultra-High Vacuum (UHV), reaching an ultimate vacuum of $2 \times 10^{-10}$ torr. 


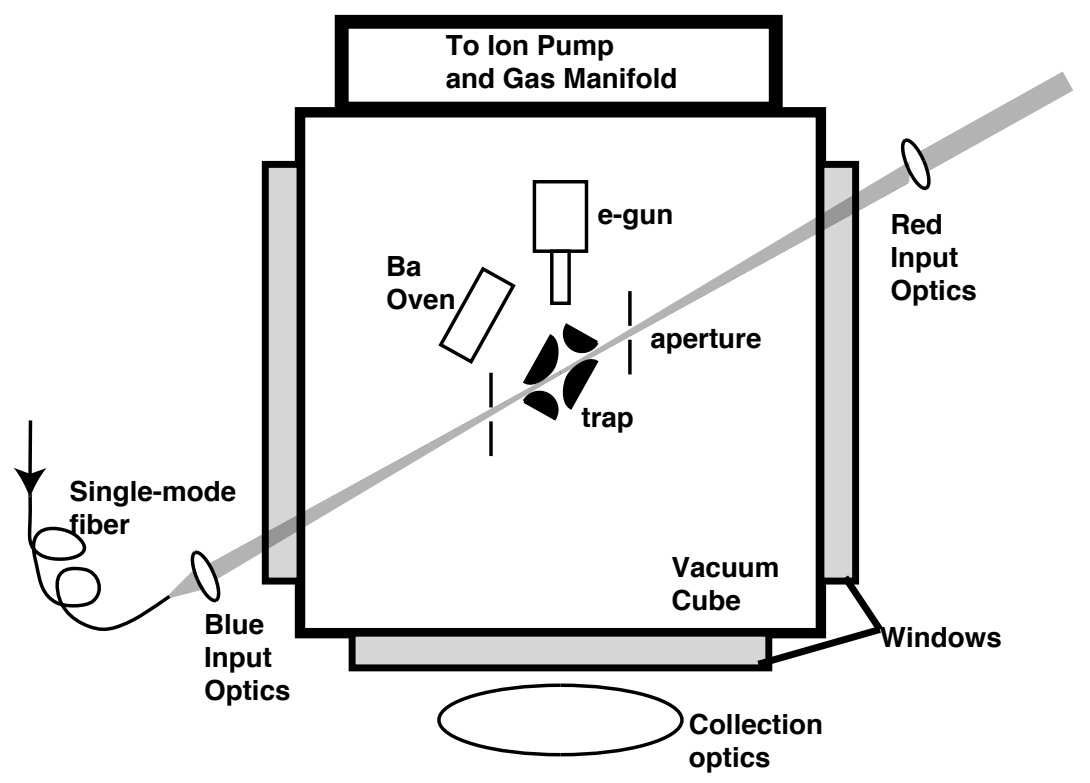

Figure 6.1: The basic experiment setup showing the ion trap and vacuum system.

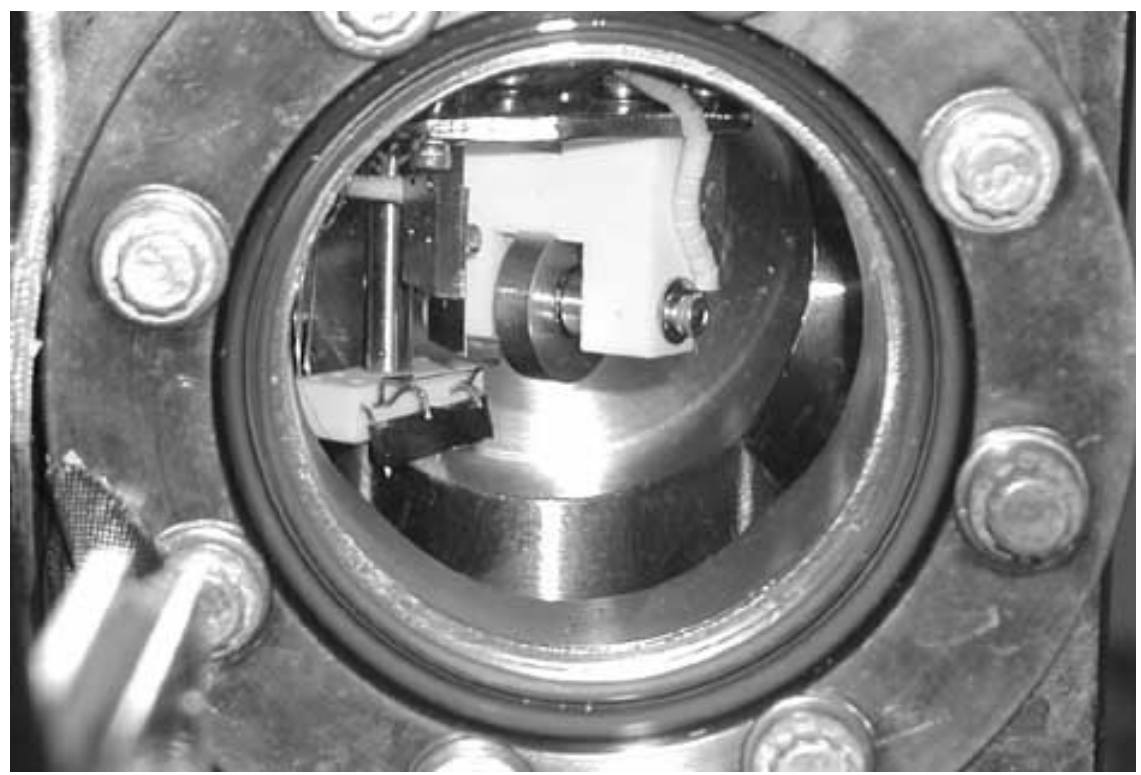

Figure 6.2: The microscope's view of the ion trap. The trap is mounted in the center of a 4.5 in. vacuum cube, with the tantalum foil barium oven to the lower left. The electron gun is located directly behind the trap. The microscope mounts on the rail in the lower left corner of the image. 


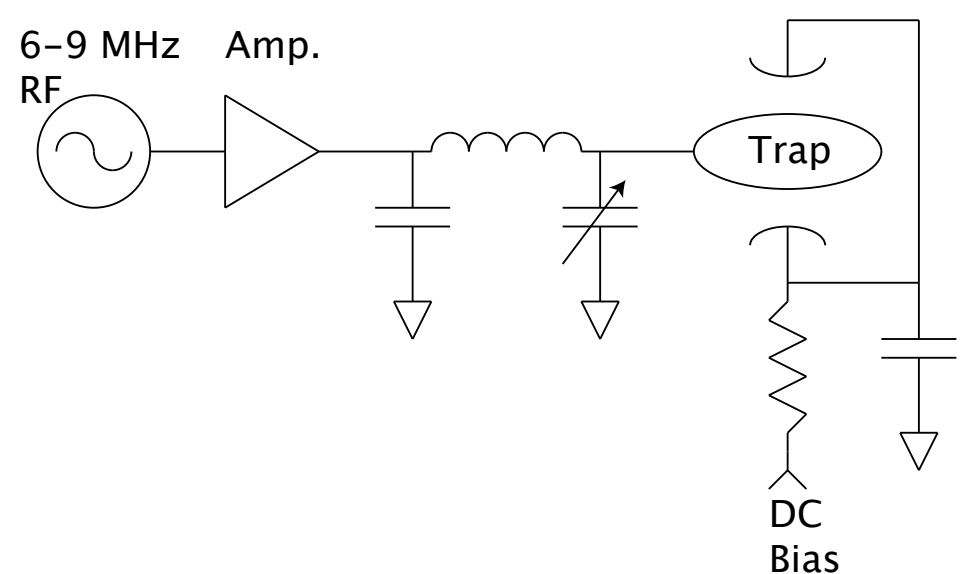

Figure 6.3: Schematic of the tuned RF resonant circuit used to generate the $2.4 \mathrm{kV}$ required for ion trapping. Not shown in the figure is a $0.3 \mathrm{pF}$ to $120 \mathrm{pF}$ capacitive divider and a directional RF power meter, both of which are used to monitor the RF voltage.

The vacuum tank connects via a metal seal valve to a bakeable gas manifold equipped with a turbo pump and oil-free scroll pump. The turbo pump is used to rough out the gas manifold to $<10^{-8}$ torr, and can also be used as a roughing pump for the vacuum tank. Helium, argon, krypton, and xenon connect to the gas manifold through a SAES hot zirconium getter which removes reactive impurities. A standard, Varian-style variable leak valve controls the flow of gas into the vacuum tank. Three gauges monitor the large range of pressures within the vacuum tank: an MKS I-Mag magnetron ionization gauge, a Stanford Research System Residual Gas Analyzer (RGA), and an extended range Pirani gauge.

A computer controlled function generator drives a $200 \mathrm{~W}$ wideband amplifier to create the RF trapping voltage. As shown in Fig. 6.3, a resonant PI-network consisting of a high-voltage RF capacitor, hand-wound air-core inductor, and a variable capacitor matches the $50 \Omega$ amplifier output to the $\sim 12 \mathrm{pF}$ trap capacitance. The inductor and trap capacitance form an $\mathrm{LC}$ resonator with $Q=30$, reducing the required RF power to manageable levels (about $15 \mathrm{~W}$ ) while restricting the operating frequency range to $6.7-9.0 \mathrm{MHz}$. A variable $\mathrm{RF}$ attenuator adjusts the trapping voltage from 0 to $700 V_{p k}$. Unfortunately, the resonant circuit cannot handle the high 
voltage required to unload the trap at high $q_{z}$, limiting the trap's mass selectivity. A power supply connected to the endcaps through a low pass filter provides the DC bias voltage used to control the $a$ parameter and consequently, the trap stability.

A frequency doubled, 986nm diode laser from Toptica Photonics generates the 493nm blue laser light while an inexpensive, custom-built 650nm laser diode provides the red. Both lasers are servoed to Fabry-Perot resonators for frequency control and stability and pass through independent acousto-optic modulators for amplitude control. The laser frequencies are measured with a Burleigh WA-1000 wavemeter with an accuracy of $\pm 500 \mathrm{MHz}$. A single mode fiber spatially filters the blue laser and transports a near-ideal beam to the delivery optics. The fiber also decouples the trap optics from the rest of the laser system. After exiting the fiber, the blue laser beam passes through a fixed polarizer and is focused into the trap center. The red laser beam is delivered in free space and passes through a tunable polarizer before focusing through the trap center, counter-propagating against the blue laser. Three pairs of magnet coils are mounted on the cube faces to control the magnetic field.

We use several techniques to detect fluorescence from the ions. When a new trap is first commissioned, the lasers and microscope are not optimally focused on the trap center and some alignment is necessary to maximize the signal. For a variety of reasons, the human eye sees small ion signals more efficiently than electronic techniques and so ions are first detected by eye. This surprising fact seems to result from eyebrain-hand coordination; small ion signals are found by toggling the red laser while looking into the trap. Visual inspection, with its combination of fast response and its sensitivity to coordinated changes, seems to be particularly good at distinguishing the toggled signals from constant background. Unfortunately, signals from the human eye are notoriously difficult to calibrate and quantify, so visual observation is only used during initial signal tune up. Once the lasers and microscope are correctly aligned, the fluorescence light is measured with a Photo-Multiplier Tube (PMT), an imaging PMT or a CCD camera mounted at the microscope image plane. A mirror diverter selects between the PMT and CCD and a blue-pass filter blocks the scattered red light. The PMT, a Hamamatsu model R7400U with 3/8 inch photocathode, is apertured to block background light from the electrodes and operates in single photon 
counting mode. The imaging PMT, built by Quantar Technology, uses a combination of microchannel plates and a resistive anode to allow single photon counting with good position resolution and microsecond timing resolution. The Photometrics CoolSnap ES CCD camera image can be cropped to reduce background, a significant advantage over the PMT, however the CCD readout takes $30 \mathrm{~ms}$ and the frame-based imaging forfeits fast timing information.

Ion trap loading is the most complicated aspect of the ion trap operation, and the least well understood. In UHV, the trap is loaded by raising the oven temperature to $\geq 370 \mathrm{C}$, then pulsing the electron gun for 2 to 10 seconds at 5 to 100 microamps. Both the red and blue laser must be illuminating the trap center slightly below resonance in order to Doppler cool the newly formed, hot ions and prevent them from unloading. The trap will not load in UHV without the appropriate laser cooling. The UHV loading process never loads more than $\sim 10$ ions. This ion number limit most likely arises from the equilibrium between ionizing neutral barium from the vapor and ionizing Ba-II from the trapped population to create Ba-III. However, the ion number limit may also arise because Doppler cooling becomes inefficient for large ion clouds and more than $\sim 10$ trapped ions are RF heated out of the trap. In UHV, the only available cooling mechanism is Doppler cooling with the Ba-II 493nm transition. Since the Ba-II does not remain trapped without laser cooling, we believe that ions other than Ba-II are unstable in the trap in UHV and consequently cannot be simultaneously trapped with barium. Further indirect evidence supports this assertion since we find no contribution to the ion trap dynamics from spectator ions.

In helium at $10^{-6}$ torr, the trap can be loaded by running the oven at $340 \mathrm{C}$ and then running the electron gun as in UHV. After the electron gun firing, an initially small ion signal steadily grows for several minutes until reaching a constant value consistent with several hundred to several thousand trapped ions. The signal increase stops when the oven is turned off and the signal disappears when the RF is cycled, indicating that barium is ionized by a trapped, invisible ion species. We believe the electron gun creates a large cloud of Ba-III which is stably trapped with the assistance of buffer gas cooling. The neutral barium is ionized by the trapped Ba-III, becoming trapped itself until all of the Ba-III has been reduced to Ba-II or until the barium 
oven is turned off. Unfortunately, we cannot rule out the possibility that an ion other than Ba-III is trapped and responsible for the gradual signal increase.

At pressure above $5 \times 10^{-6}$ torr, the ion pump creates a plasma which loads the ion trap. The exact mechanism of this "ion pump loading" defies explanation; although the ion pump has a direct line of sight into the vacuum chamber, a grounded grid covers the mouth of the pump to prevent plasma from entering the trap region. The $\sim 20 \mathrm{eV}$ trap depth should also prevent any ions from entering the trap unless those ions have very high energy and are cooled while they cross the trapping volume. Finally, there is very limited access to the trap volume between the electrodes and the probability of loading by accident seems negligible. Nonetheless, at high pressure ion pump loading very effectively fills the ion trap with barium ions.

\subsection{Hyperbolic trap in vacuum}

\subsubsection{Signal quantization}

Signal quantization is the first measurement of any self-respecting ion trapper. In order to measure a single ion signal, ions are unloaded (or in some cases, loaded) in a controlled fashion such that each removed ion decreases the total fluorescence signal in integer steps. This methodology shares much in common with the Millikan oil drop experiment, and has similar draw backs. Most notably, for a given series of quantized steps, each step could correspond to more than one ion unloading at each step. And much like Millikan's experiment, the trapped ion dynamics and fluorescence signal are subject to fluctuations (crystallization, chaotic behavior, etc.) that contribute uncertainty to the results. In our case, the quantization measurements have been repeated with different traps, detectors, and optics, with consistent results throughout in spite of the sporadic fluctuations that we attribute to complex trap dynamics.

To generate the quantized steps show in Fig. 6.4, we prepare a small cloud of ions by gas-loading in $10^{-6}$ torr helium and then pumping down to $10^{-9}$ torr with the ion pump. The blue laser is slightly de-tuned to provide laser cooling, and ion species other than Ba-II are assumed to RF heat out of the trap. While recording the ion 
fluorescence, the trap depth is lowered by cycling the DC bias voltage from $0.0 \mathrm{~V}$ to $4.0 \mathrm{~V}$ for one second every 20 seconds. When the trap depth is low, the hottest ions from the ion cloud have a small chance, about 1 in 7 , to "evaporate" out of the trap before the trap returns to its initial state. Under computer control, the bias voltage cycles 40 times over 20 minutes while the PMT records the fluorescence.

The fluorescence measurements collected when the bias voltage is high are removed from the time series shown in Fig. 6.4(a). The remaining spurious low points are the result of transient behavior near the bias voltage step and are not removed from the results. The time series is histogrammed in Fig. 6.4(b) with each peak fit to a Gaussian distribution. The Gaussian intervals are chosen to span each peak with the broadest reasonable range. Note that the "half-step" with amplitude $2.6 \mathrm{kHz}$ from sample 560 to 580 is included in the histogram unaltered, yet clearly stands out as a case in which the trapped ion dynamics alters the signal. Each Gaussian peak is associated with an ion number, starting with zero ions for the $1.8 \mathrm{kHz}$ background samples (measured with the RF off) and increasing to 6 ions. The Gaussian means are then plotted against the integer number in Fig. 6.4(c) and fit to a linear polynomial. Note that the graph includes the error-bars for the uncertainty in the means. The fitted signal per ion is $610 \pm 13$ photoelectrons per second.

\subsubsection{Ion fluorescence spectrum}

In UHV, the ion fluorescence rate strongly depends on the laser frequency detuning and laser power. Without the velocity damping provided by buffer gases, trapped ions heat up and exit the trap within seconds unless the both the blue and red lasers are red-detuned just below resonance. This makes taking spectra in UHV difficult since tuning the lasers too far to the blue side of the transition will immediately unload the ions. Similarly, tuning the lasers too far to the red side of the transition reduces the laser cooling power and the ions unload. Many experiments avoid this difficulty by probing the velocity distribution with a low power laser on a third transition while cooling the ion with a laser on the strong transition. Unfortunately, the metastable levels of barium require two additional lasers to implement such a scheme, a luxury 

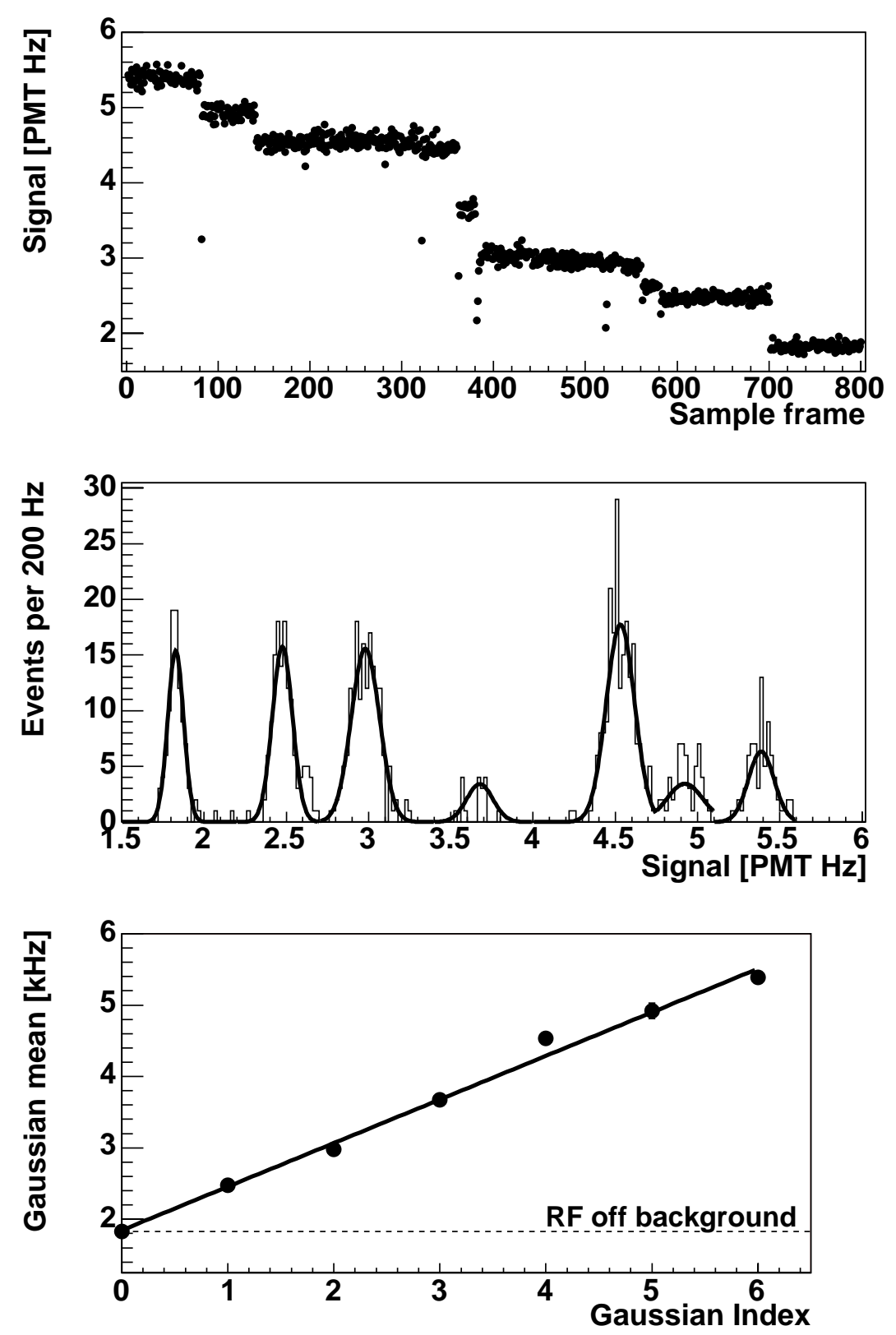

Figure 6.4: A Millikan "ion drop" experiment showing quantization of the ion signal. (a) Time series of a trapped ion cloud unloading. Every 20 samples, the trapping potential is reduced and with low probability, a single ion leaks out of the trap. (b) Histogram of the signal amplitudes with Gaussian fits. (c) Linear fit of the Gaussian means to the suspected ion number. Each ion generates PMT photoelectrons at $610 \pm 13 \mathrm{~Hz}$. 
unavailable at the time of this thesis.

In spite of the UHV unloading instability, spectra are regularly taken by fixing the blue laser frequency slightly below the fluorescence maximum and sweeping the red laser frequency from $\sim 500 \mathrm{MHz}$ below the resonance to just above the peak of the signal. Fig. 6.5(a) contains a typical red fluorescence spectrum sampled at $5 \times 10^{-10}$ torr. Based on the total fluorescence signal and the ions' qualitative behavior, we infer that two ions were trapped for this spectrum. The physical size of the ion cloud, measured by fitting the ion image to a 2-D Gaussian, is shown in Fig. 6.5(b). As the red frequency is increased, the signal demonstrates three general features of UHV fluorescence:

- $\sim 200 \mathrm{MHz}$ below the resonance peak, the signal drops suddenly and the ion cloud gets smaller,

- the fluorescence intensity drops sharply when the red and blue detunings are equal, and

- slightly above the peak fluorescence, the ion cloud "explodes", increasing in size and decreasing in brightness.

The cloud contraction and explosion result from doppler cooling of the ions; as the red laser detuning approaches resonance, the cooling efficiency improves, damping the ions' velocities until they condense into a crystal. Above the resonance, doppler cooling turns into doppler heating, the ion crystal "melts", and the ions gain energy until they are ejected from the trap or from the laser beams. The decrease in fluorescence at $515 \mathrm{MHz}$ occurs when the red and blue laser detunings are equal and arises from the two-photon resonance discussed in $§ 3.5$.

\subsection{Trap asymmetry and RF micromotion}

Asymmetries in the ion trap construction, patch voltages on the electrodes, and contact potentials from barium plating create a DC bias voltage across the trapping region. Uncompensated, the bias voltage shifts the potential minimum away from the 


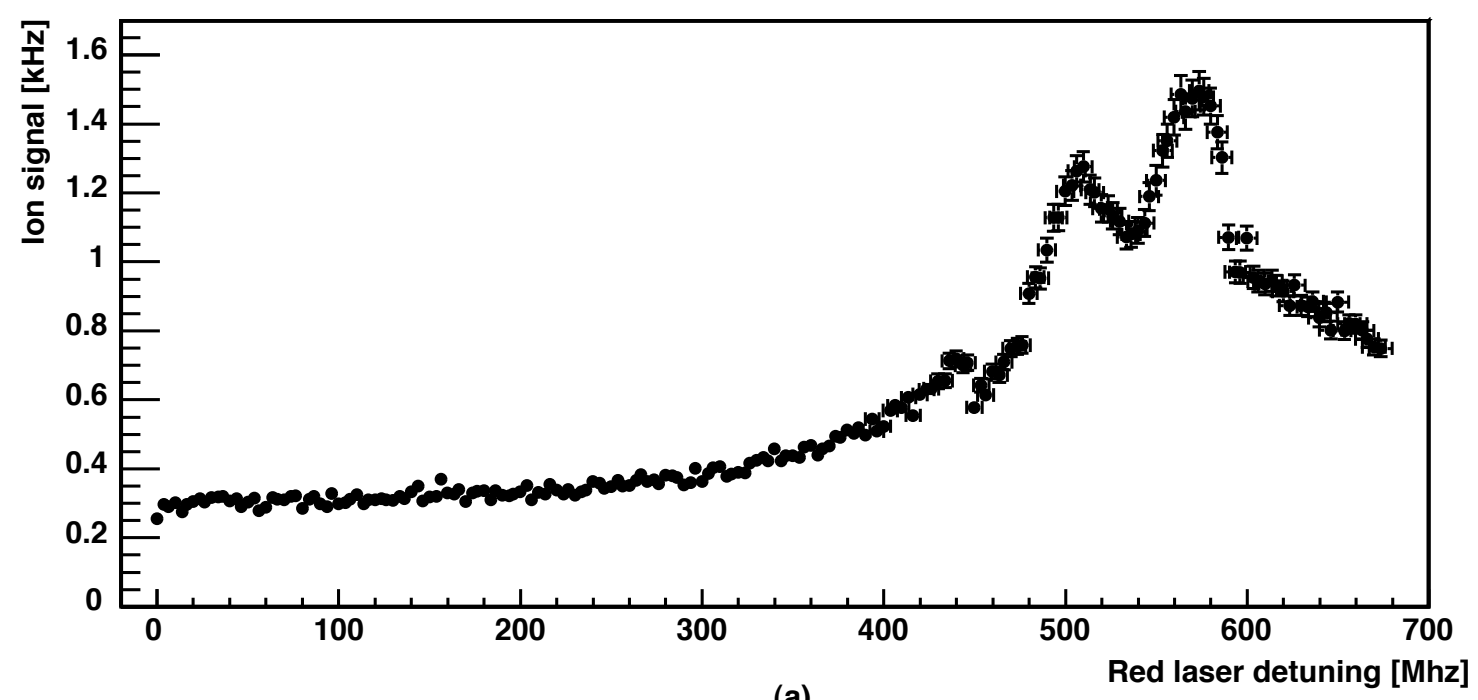

(a)

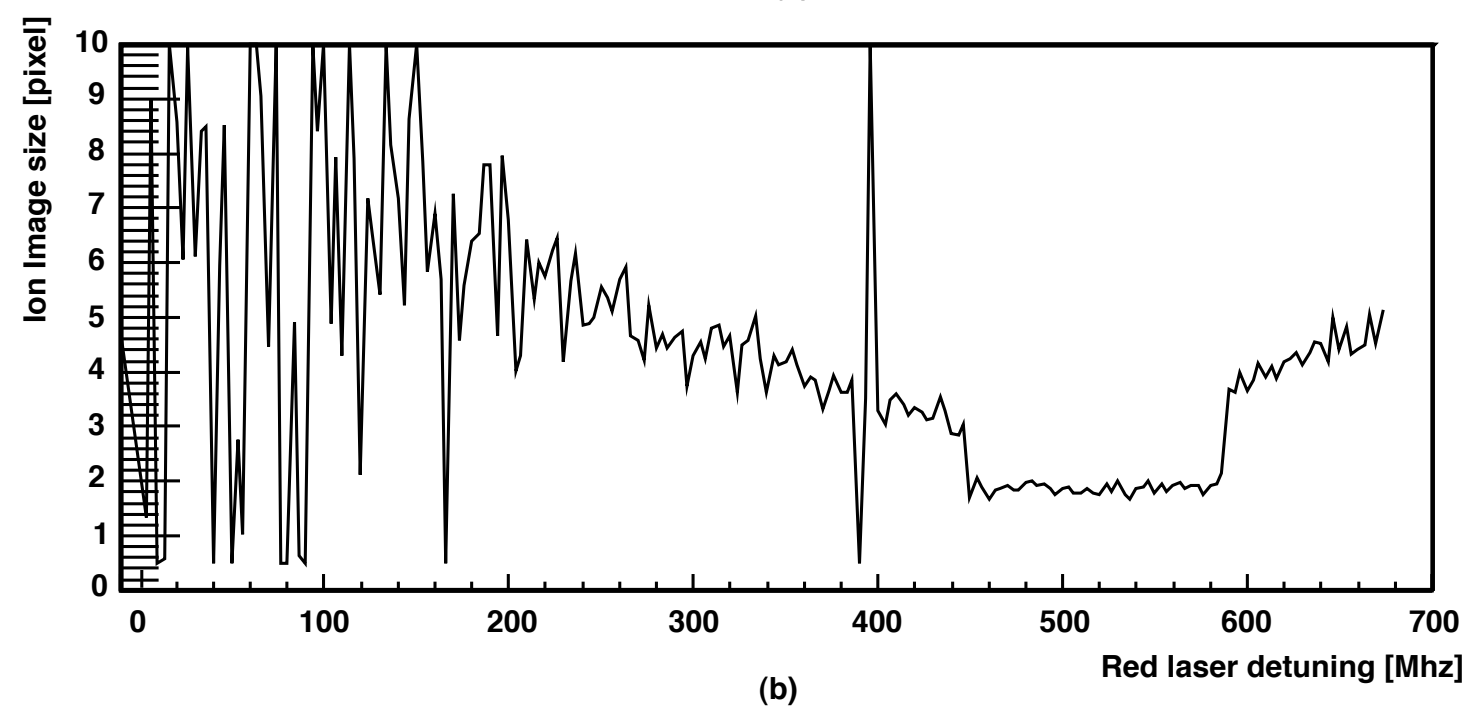

Figure 6.5: (a) Resonance spectrum of two ions with fixed blue detuning and variable red detuning. The spectrum shows features of doppler cooling, two photon resonance, and doppler heating as discussed in the text. (b) Width of the ion image showing "condensation" of ions into a cold crystal at $450 \mathrm{MHz}$ and heating at $580 \mathrm{MHz}$. 
trap center to a region of non-zero average RF field, greatly increasing the macromotion. In addition to the increased micromotion, the non-ideal potential increases the $\mathrm{RF}$ heating, requiring constant laser cooling in order to maintain a trapped ion. As discussed in $§ 3.4$, the micromotion frequency modulates the absorption profile. Without an absolute frequency standard with which to measure the modulation depth and frequency shift, a quantitative fit of the three-level OBEs to the spectra in Fig. 6.5 can't be done.

The ion cloud behavior as a function of the trapping voltages provides a convenient probe of the bias voltage in the trap. Fig. 6.6(a) shows the shift of the ion cloud center as a function of the RF voltage. As the RF potential decreases, the minimum of the combined RF and DC bias potentials shifts along the y-axis. Indeed, the y-axis should have a large asymmetry due to the the placement of the barium oven and resulting plating asymmetry, the only known electrode asymmetry from a pair of mounting holes in the ring electrode, and the coincident alignment of the y-axis with gravity. The ion clouds imaged in Figs. 6.6(b) and (c) vividly demonstrate the effect of the trap asymmetries on the ion oscillation eigenmodes. In an ideal trap, the oscillation modes along the $x$ and $y$ axes have the same frequency and efficiently couple to each other; a single laser cooling one of the radial axes cools the other radial axis as well. The trap asymmetry lifts the degeneracy between the $x$ and $y$ axes so that the radial axes have different oscillation frequencies and must be cooled independently. By coincidence, the cooling laser enters our ion trap at an angle between the $x$ and $z$ axes with no component along the y axis. Thus the $x$ and $z$ motions are well cooled while the $y$ motion is left uncooled, leading to the cigar shaped ion cloud shown in Fig. 6.6(b). In Fig. 6.6(c), additional ions were added to the trapped cloud of (b). With more than one ion in the trap, coulomb interactions couple the motions along the $x$ and $y$ axes and all three motions are cooled.

A set of external bias electrodes could compensate for the DC bias. Unfortunately, our hyperbolic electrode geometry effectively shields the trap center and compensation electrodes would have to be placed very close to the gap between the ring and end-caps in order to generate sufficient field at the trap center. Since those same electrodes lower the breakdown voltage in buffer gases, we elected not to include them. 


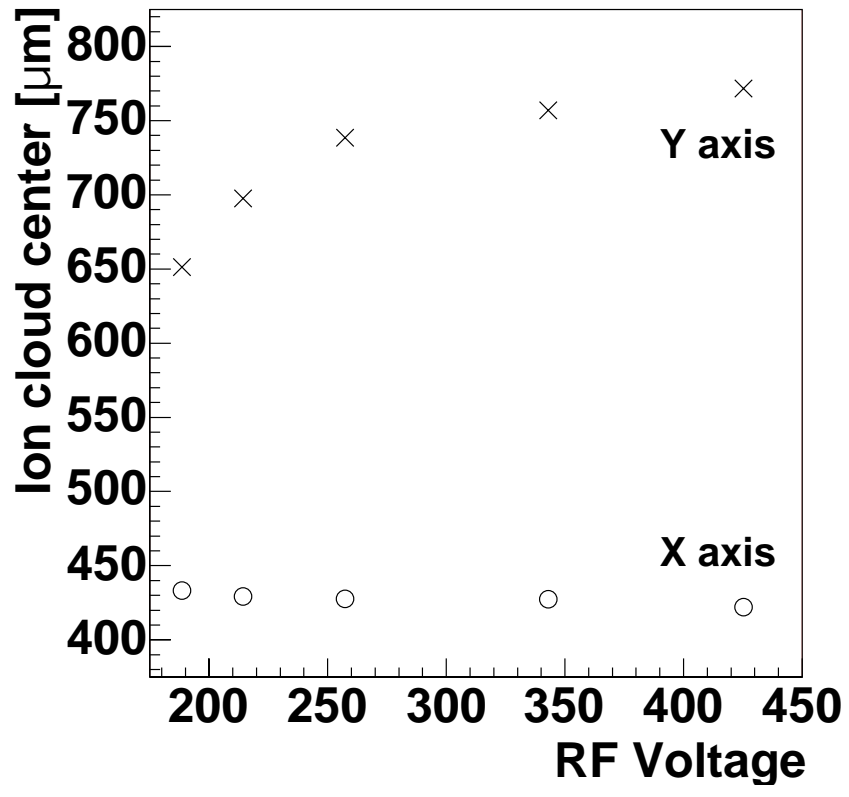

(a)

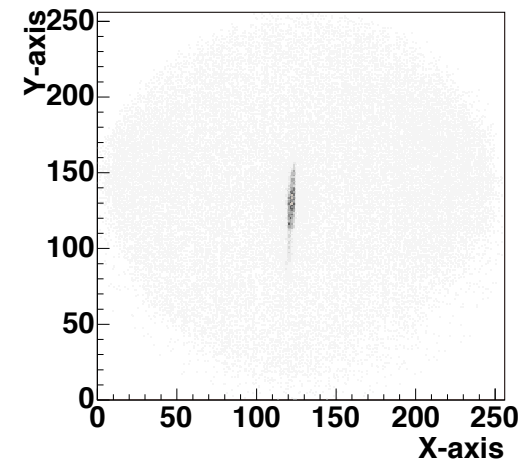

(b)

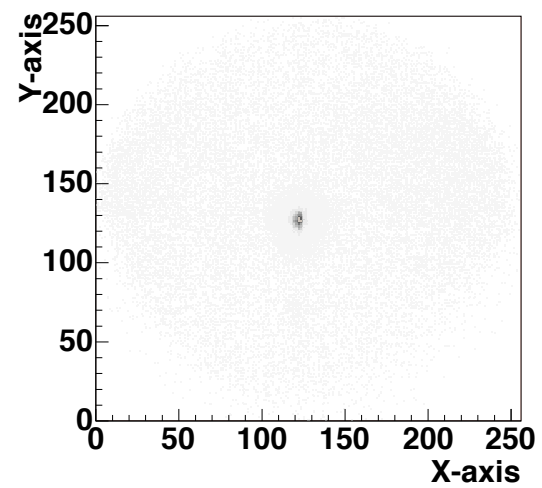

(c)

Figure 6.6: Several manifestations of trap asymmetry. (a) The cloud center shifts along the $y$-axis $(x)$ while remaining stationary on the $x$-axis $(0)$ as the RF voltage increases, evidence of a DC-bias along the y-axis. (b) With one ion in the trap, the ion cloud elongates along the y-axis. (c) With multiple ions, coulomb interactions couple the axes of motion and the cloud becomes symmetric. See text for further discussion. 


\subsection{Hyperbolic trap in gas}

The ion signal character changes dramatically as the gas pressure increases. Most importantly, velocity damping in the buffer gas obviates the need for laser doppler cooling. The buffer gas also alters the spatial and velocity distributions, and, as already discussed, greatly improves the loading efficiency. A substantial background of helium or argon gas simplifies all aspects of the trap operation.

\subsubsection{Buffer gas filling}

The simplest method of introducing buffer gas into the trap is to turn off the ion pump and allow the system pressure to increase. The ion pump has pumped so much helium over its lifetime that it outgasses helium to $\sim 10^{-6}$ torr over thirty minutes. The resulting gas spectrum has been measured with the Residual Gas Analyzer (RGA) and found to consist mostly of helium. Conveniently, this gas filling technique does not introduce contaminants into the vacuum system; the gas is "clean". Unfortunately, the vacuum gauges act as miniature getter pumps when operated and will hold the system pressure below $10^{-7}$ torr. Consequently, it is difficult to make quantitative measurements of the pressure dependent behavior using this technique since the gauges must be turned off.

Another technique to add buffer gas to the vacuum tank is to bleed-in gas from the manifold without turning off the ion pumps. This technique has the advantage of being very controllable since the pumps provide a repeatable restoring force. The ion pump also acts to clean the incoming gas by efficiently pumping reactive impurities and reducing the chance that the impurities react with trapped barium. In order to maximize the pumping speed in the vacuum tank, the ion pump opens with a direct line of sight into the vacuum cube. As discussed in $\S 6.1$, at pressures above $5 \times 10^{-6}$ torr, a plasma develops in the ion pump that loads barium ions into the ion trap, greatly confusing fluorescence measurements.

The only technique suitable to attain buffer gas pressures above $10^{-5}$ torr requires shutting off the ion pump to avoid loading. Impurities in the gas are not filtered by the pump and consequently, this procedure critically depends on the initial gas purity. 
Trace gases present in concentrations below the RGA sensitivity react with the ions and cause the trapped ion signal to fade away. Signal decay time constants of 0.5 to 2 hours are common when the gas purification is incomplete. However, with sufficiently high gas purity, the ion signal lasts indefinitely. Nitrogen, helium, argon, krypton, and xenon have all been used as buffer gases.

\subsubsection{Spatial distribution}

Helium buffer gas modifies the ion cloud distribution within the ion trap, even at very low pressures. Fig. 6.7 displays our first quantitative gas trapping results in helium. Using an imaging PMT for fluorescence detection, trapped ions are imaged as the pressure is raised from $3.0 \times 10^{-9}$ torr (Fig. $6.7(\mathrm{a})$ ) to $4.2 \times 10^{-5}$ torr helium (Fig. 6.7(d)) with the ion pump running. In this run there was no evidence of ion pump loading. At the two lowest pressures, the ions are laser cooled and condense in the trap center to create a localized intensity peak. At $3.9 \times 10^{-7}$ torr (Fig. 6.7(c)), the gas collisions overcome the laser cooling and the cloud dissipates into a somewhat larger ion cloud without the crystallized center. As the pressure increases to $4.2 \times 10^{-5}$ torr (Fig. 6.7(d)), collisions completely dominate the trap dynamics and the ion cloud becomes elongated and diffuse. In the "high" pressure image, the ion cloud image is determined by the $55 \mu \mathrm{m}$ laser beam waist in the vertical dimension and by the gap between the electrodes in the horizontal direction. The helium gas collisions also provide velocity damping, so the ions are stably trapped even when the laser cooling is turned off.

As the helium pressure increases, the ion cloud gradually contracts and concentrates in the trap center, increasing the ion signal. Fig. 6.8 shows the effect on the PMT signal of increasing the pressure from $10^{-6}$ to $10^{-1}$ torr and then lowering the pressure to $10^{-6}$ torr using the turbo pump. The signal increase is not a result of a change in the number ions, but rather is a consequence of the modified cloud distribution. The increase in signal plateaus when the majority of the ion cloud is contained within the lasers and the field of view. Fig. 6.9 shows CCD images of an ion cloud taken at (a) $10^{-5}$ and (b) $10^{-2}$ torr. The intensity scale for both images is the same, 
(a) 3.0e-09 torr

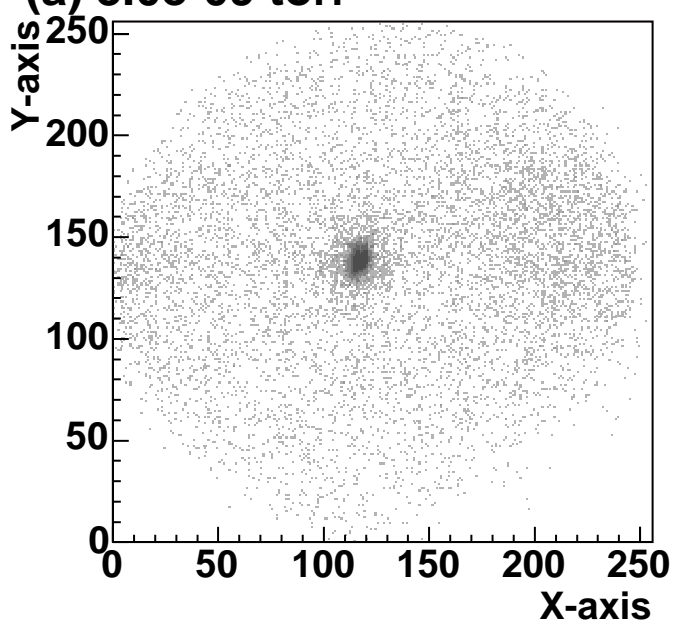

(c) 3.9e-07 torr

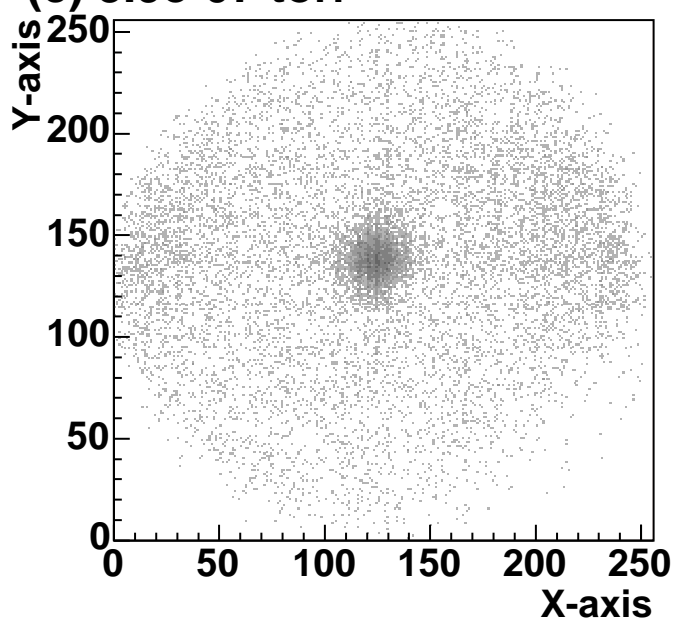

(b) $4.3 \mathrm{e}-08$ torr

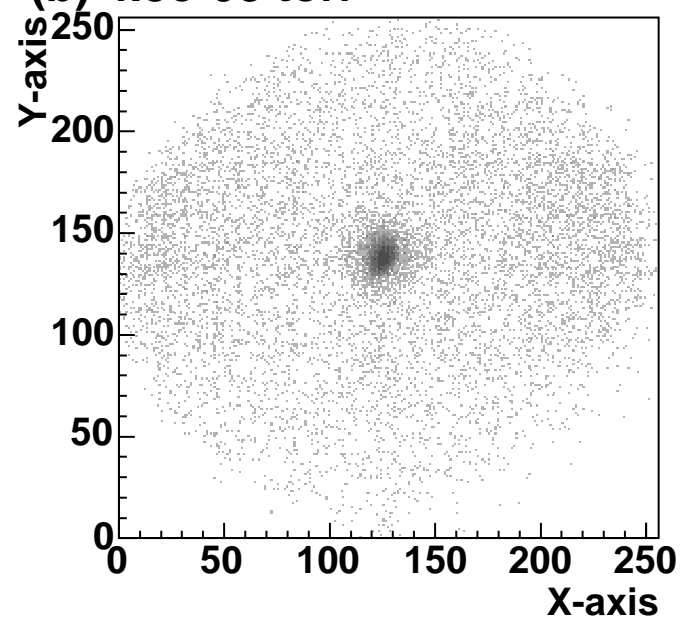

(d) 4.2e-05 torr

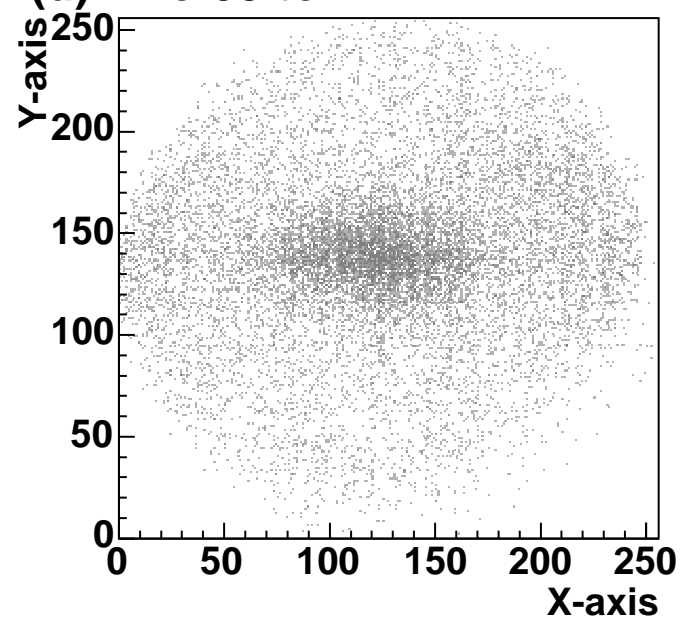

Figure 6.7: The effect of helium buffer gas on the ion cloud spatial distribution. As the pressure increases from (a) $3.0 \times 10^{-9}$ torr to (d) $4.2 \times 10^{-5}$ torr, the laser cooling stops working and the ion cloud becomes large and diffuse. 


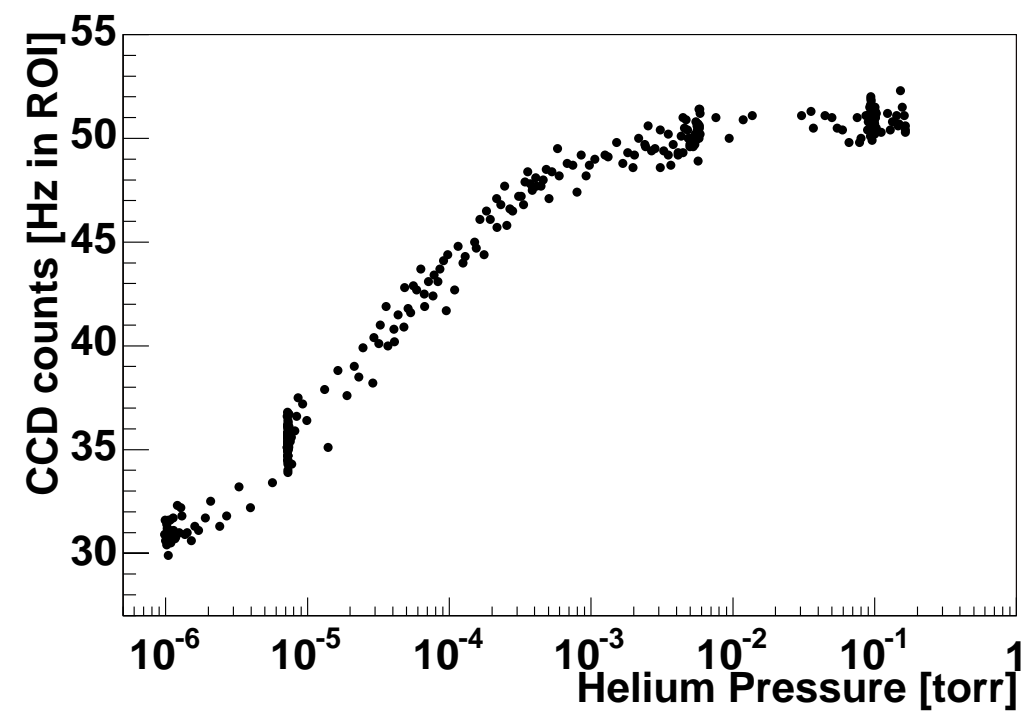

Figure 6.8: The fluorescence signal from an ion cloud as the helium pressure is swept from $10^{-6}$ torr to $10^{-1}$ torr and then back to $10^{-6}$ torr. This data was taken with an imaging PMT.

revealing the substantial increase in both the peak signal and the signal "density". Similar behavior is observed in argon.

Using the values in Table 5.2, we calculate the mean time between collisions to be 0.1 seconds at $10^{-5}$ torr, decreasing to $1 \times 10^{-4}$ seconds at $10^{-2}$ torr. The difference in the mean free time between collisions explains the change in the signal behavior in Fig. 6.9. At low pressure, the time in between ion-neutral collisions is much longer than any other time scale in the trap dynamics and so the buffer gas has limited impact on the trapped ions. On the other hand, at high pressure a buffer gas atom collides with a trapped ion every few macromotion periods, efficiently damping the macromotion and collecting the ions in the trap center. 


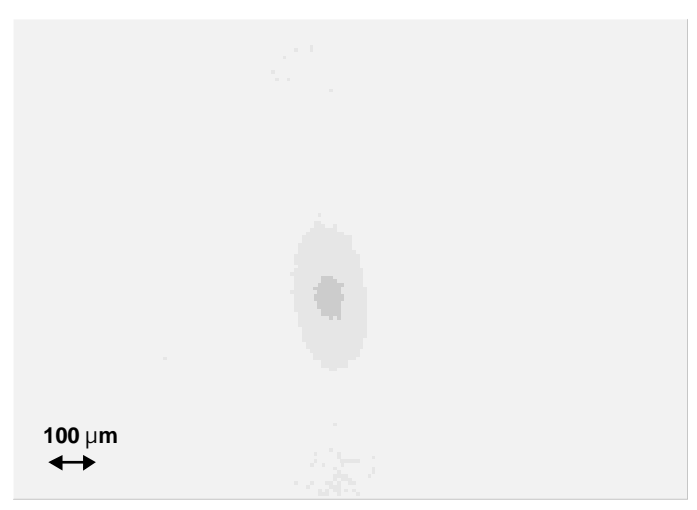

(a) $10^{-5}$ torr $\mathrm{He}$

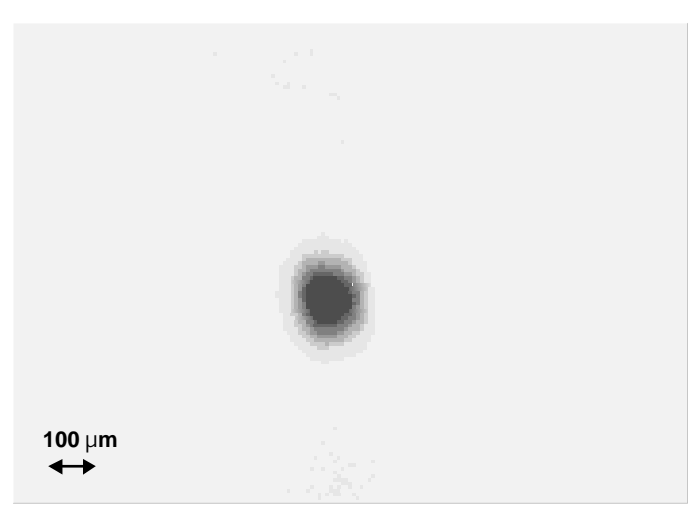

(b) $10^{-2}$ torr $\mathrm{He}$

Figure 6.9: A single cloud of ions at (a) $10^{-5}$ and (b) $10^{-2}$ torr, measured using a cooled CCD. The intensity scale is the same for both graphs.

\subsubsection{Buffer gas spectra}

Resonant fluorescence spectra directly probe the velocity distribution of the trapped ions and can lend insight into the different cloud behaviors. As shown in Fig. 6.10, the spectrum for the ion cloud at $6.2 \times 10^{-2}$ torr is identical to the spectrum at $6.9 \times 10^{-5}$ torr and to the spectra at intermediate pressures. Consequently, these doppler spectra do not directly display a difference in the ion motion responsible for the signal increase with pressure. Nonetheless, Fig. 6.10 interests us for several additional reasons.

1. The spectrum is taken by scanning the blue laser, and no evidence of heating can be seen.

2. The two photon resonance has all but disappeared, though high resolution scans do detect it as a small signal dip.

3. The width of the resonance has increased substantially from similar spectra in UHV such as Fig. 6.5.

4. There is no evidence of the signal increase seen in Fig. 6.7. This may result from the increase in RF frequency from 8.5 to $9.99 \mathrm{MHz}$, or it may result because far fewer ions are trapped. 


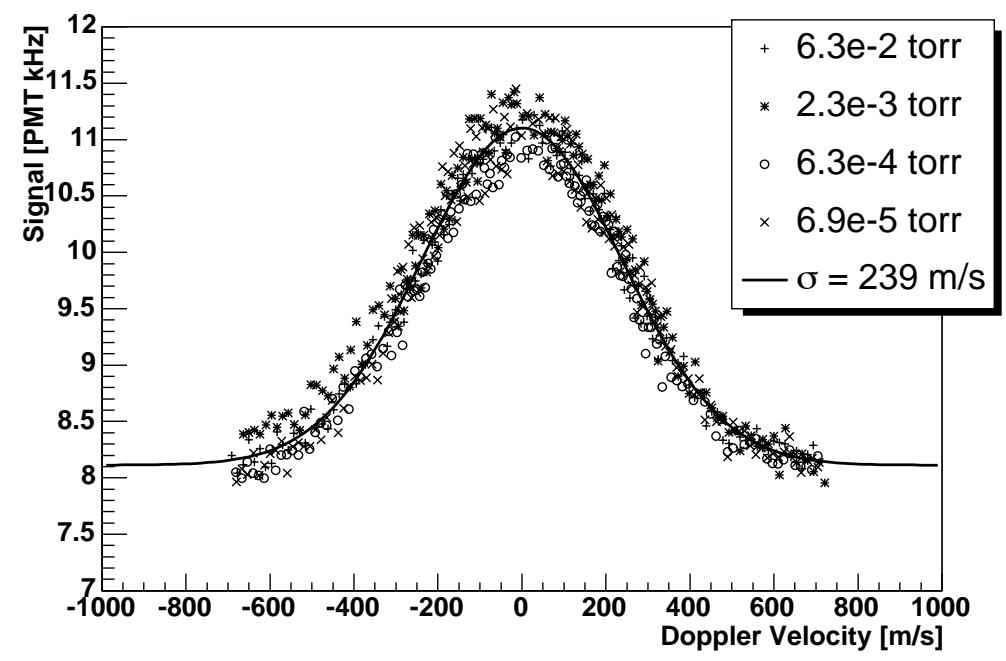

Figure 6.10: Blue fluorescence spectra for ions in $6.9 \times 10^{-5}$ to $6.3 \times 10^{-2}$ torr helium buffer gas. The spectra are fit to a Gaussian profile with $\sigma=239 \mathrm{~m} / \mathrm{s}$. These spectra were taken with $\Omega_{R F}=9.99 \mathrm{MHz}$ instead of the standard $8.5 \mathrm{MHz}$.

5. The width of the distribution taken with RF frequency $\Omega_{R F}=9.99 \mathrm{MHz}$ is the same as the width at $8.5 \mathrm{MHz}$ as shown in Figs. 6.11.

6. Finally, a Gaussian distribution fits the center of the spectrum quite well, indicating a thermal origin for the spectral width and not collisional broadening.

The wings of the distribution deviate from a Gaussian profile as a result of the Lorentzian resonance lineshape and a Voigt function must be used to properly fit the spectra as shown in the high resolution spectrum in Fig. 6.11. The Voight lineshape is given by

$$
P(\omega)=\frac{\Gamma}{\pi} \int_{-\infty}^{\infty} \frac{e^{-\omega^{2}}}{\left(\omega-\omega^{\prime}\right)^{2}+\Gamma^{2}} d \omega^{\prime}
$$

As discussed in $\S 3.4$, we expect a Gaussian resonance to result from a thermal distribution of the ions with $\sigma=\sqrt{k T / m}$. The $\sim 240 \mathrm{~m} / \mathrm{s}$ width of the spectra shown in Figs. 6.10, 6.11, and 6.12 corresponds to a temperature of $948 \mathrm{~K}$. The velocity distribution measured via the blue resonance (Fig. 6.10) is identical to that measured with the red resonance (Fig. 6.11), indicating that no systematic errors in 


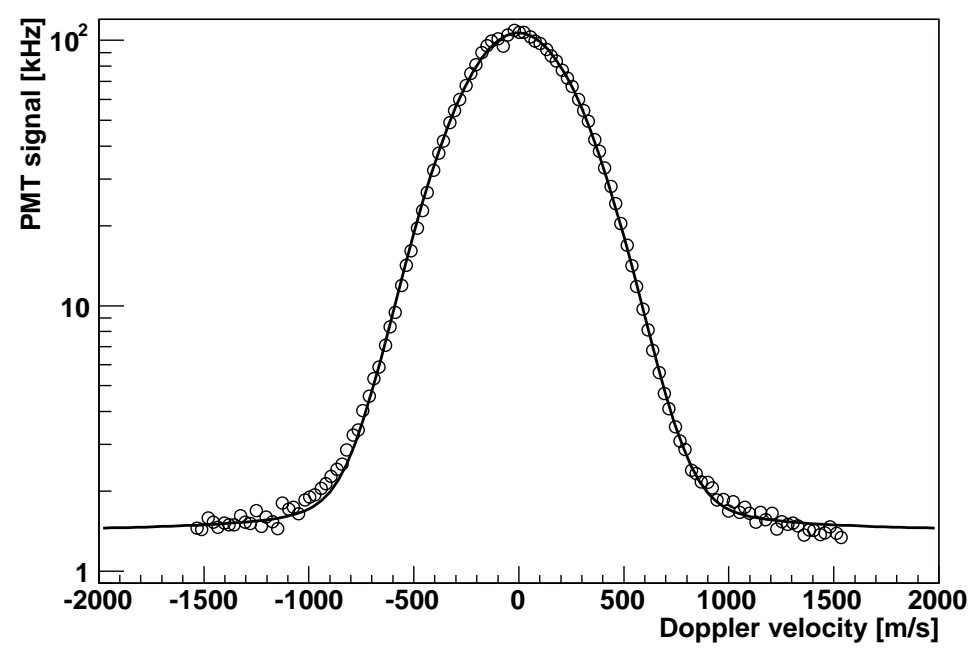

Figure 6.11: Red fluorescence spectrum for ions in $1.3 \times 10^{-1}$ torr helium fit to a Voight profile. The profile fits the wings of the distribution better than a Gaussian. Note the logarithmic scale. The fit has $\sigma=257 \mathrm{~m} / \mathrm{s}$ and $\Gamma=17.2 \mathrm{~m} / \mathrm{s}$

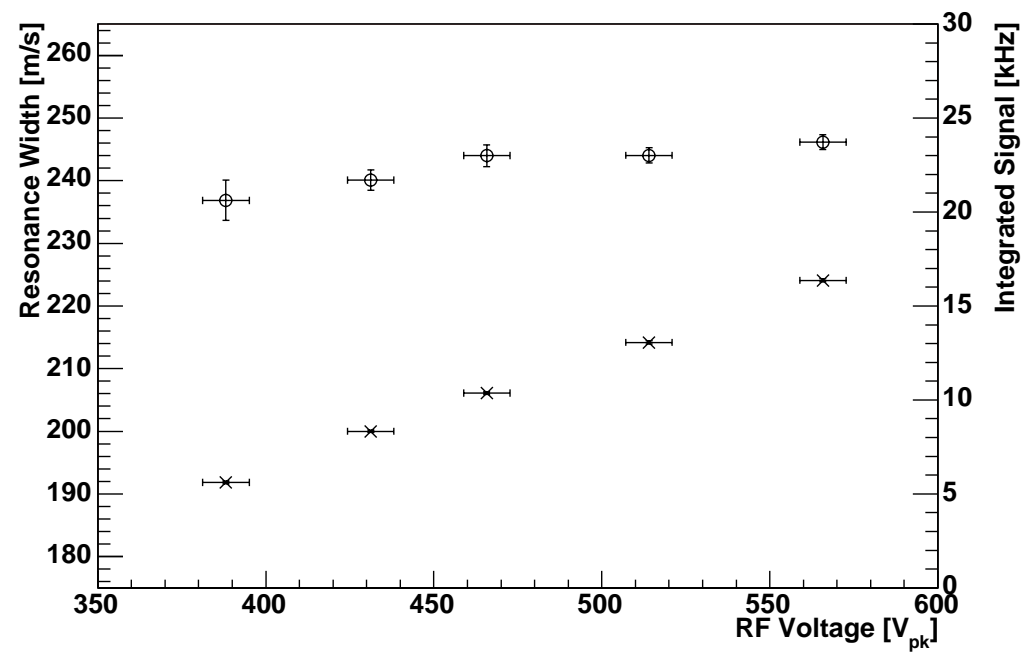

Figure 6.12: Results of resonance spectra taken in $1 \mathrm{~m}$ Torr helium at a range of $\mathrm{RF}$ voltages. Gaussian $\sigma$ are shown (o) with a mean value of $243 \mathrm{~m} / \mathrm{s}$ and a slight slope. Also shown $(x)$ is the integrated signal for each spectrum with a linear dependence of $59.2 \pm 4.5 \mathrm{~Hz} / \mathrm{V}$. 
the laser frequencies contribute to the observed high temperature. Naively, we expect the high temperature to result from an equilibrium between RF heating and buffer gas cooling. Somewhat surprisingly, the resonance width does not depend on the RF parameters as shown in Fig. 6.12. In this measurement, the RF voltage is varied from the standard trapping value, $570 \mathrm{~V}$, to the minimum trapping value, $380 \mathrm{~V}$, in discrete steps. At each step, the blue laser frequency sweeps through the ion resonance and the signal is recorded. The resulting spectra are fit to a Gaussian lineshape and the $\sigma$ and integrated signal are plotted in Fig. 6.12. The Gaussian width slightly correlates with the RF voltage, with a mean of $243 \mathrm{~m} / \mathrm{s}$ and voltage dependence of $.05 \mathrm{~m} / \mathrm{s} / \mathrm{V}$. On the other hand, the signal magnitude shows a strong dependence on the $\mathrm{RF}$ voltage with a slope of $59.2 \pm 4.5 \mathrm{~Hz} / \mathrm{V}$. The signal-RF dependence arises from the same source as the spatial distributions discussed in §6.4.2. As the RF voltage decreases, the trap depth decreases and the ions spread out, decreasing in density. As the density decreases, the ions spend less time in the laser beams and the collection optics field of view, leading to a lower signal. However, the relative constancy of the distribution width implies that the velocity of the trapped ions does not arise directly from an equilibrium between RF heating and collisional cooling. Instead, as we will show later, the velocity distribution arises as a general consequence of collisions in an RF trap.

\subsection{Argon buffer gas}

Trapped ions in argon buffer gas behave qualitatively and quantitatively like ions in helium gas and we do not repeat all of the data here. Fig. 6.13 is representative of the argon data, showing the doppler velocity distribution for a cloud of barium ions at two buffer gas pressures: $3.2 \times 10^{-5}$ and $8.0 \times 10^{-4}$ torr. Similar spectra have been taken up to $P=1 \times 10^{-2}$ torr. The velocity distribution has the same width at both pressures, $\sigma \sim 240 \mathrm{~m} / \mathrm{s}$. A comparison with Fig. 6.10 reveals identical linewidths for spectra in helium and argon, implying that the heating mechanism is independent of the mass and collision cross-section of the buffer gas. Ions have remained trapped for several day unattended in both helium and argon gas. 


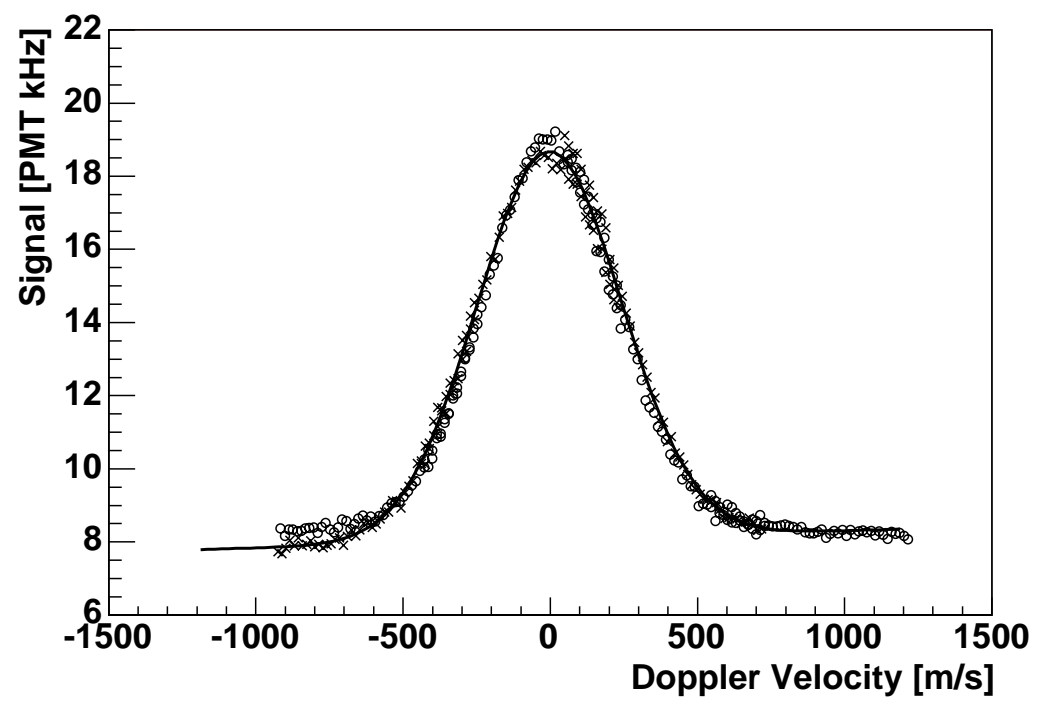

Figure 6.13: Doppler spectra in argon gas at $3.2 \times 10^{-5}$ torr $(\times)$ and $8.0 \times 10^{-3}$ torr (०). The spectra are fit to a Gaussian line shape with $\sigma=246 \mathrm{~m} / \mathrm{s}$ (solid line).

\subsection{Heavy buffer gases}

The phenomenology of Ba-II trapped in helium and argon provides invaluable information about ion dynamics in high pressure buffer gas. However, the goal of this experimental program is to measure barium trapping in xenon. Accordingly, immediately after confirming that the ion signal remains in helium, we began our investigations of trapped Ba-II in xenon gas.

The effect of xenon buffer gas on trapped barium is obscured by the ion pump loading discussed in $\S 6.1$. At first, ion pump loading was mistaken for a pressure dependent signal increase like that in helium (Fig. 6.8). However, with ion pump loading in xenon, the signal increase depends on time, not on pressure. Once we recognized the ion pump loading mechanism, all of our data was taken with the ion pump off; unless explicitly noted, all of the data presented in this work was taken with the ion pump off.

Our experiments with Ba-II in xenon all share one feature: under no circumstances can barium ions be trapped with xenon buffer gas; xenon unloads ions from the trap. 


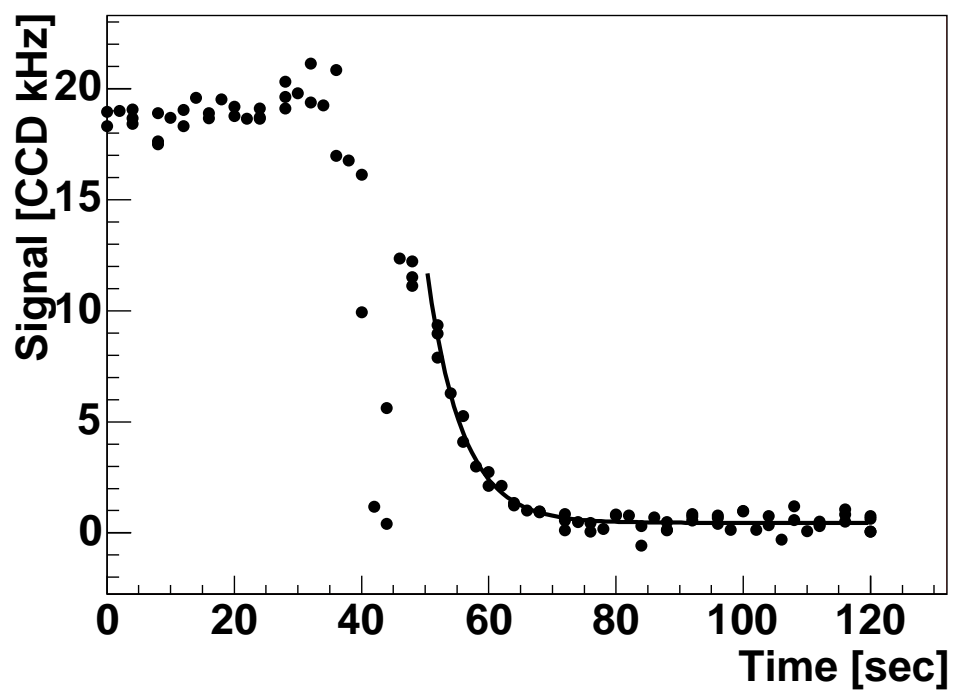

Figure 6.14: A small ion cloud is trapped in $1 \times 10^{-6}$ torr of background gas (mostly helium) until $2.0 \times 10^{-5}$ torr of xenon are introduced at $t=40 \mathrm{~s}$ when blue laser is briefly turned off to mark the beginning of the xenon fill. Then the signal decays exponentially with $\tau=5.5 \pm .07 \mathrm{~s}$ as fit by the solid line.

Fig. 6.14 shows what happens to the ion signal when we add xenon to the system. The signal from a small ion cloud, loaded at $1 \times 10^{-6}$ torr with the ion pumps off, remains constant until $5.5 \times 10^{-5}$ torr of xenon gas is introduced at $t=40 \mathrm{~s}$. Then the signal promptly fades away. An exponential decay fits the fading with time constant $\tau=5.52 \pm .07 \mathrm{~s}$. The time constant error is taken from the minimization routine assuming Poisson statistics for the measured signal rate. Note that the signal fading occurs with several xenon bottles from different sources, different regulators, and before and after multiple cleanings of the gas manifold.

The signal decay rate depends on the partial pressure of the light background gas; the higher the helium pressure in the system, the slower the signal fades away. Fig. 6.15 shows a characteristic decay signal when the trap is run with a substantial helium pressure. First, a large ion cloud is loaded and the pressure is raised to $1 \times 10^{-3}$ torr of helium with the ion pump off. After establishing a constant signal in the helium buffer from $t=0$ to80 $\mathrm{s}$, xenon is leaked into the system via the UHV leak valve. The leak valve is calibrated so that the speed of the pressure increase is 


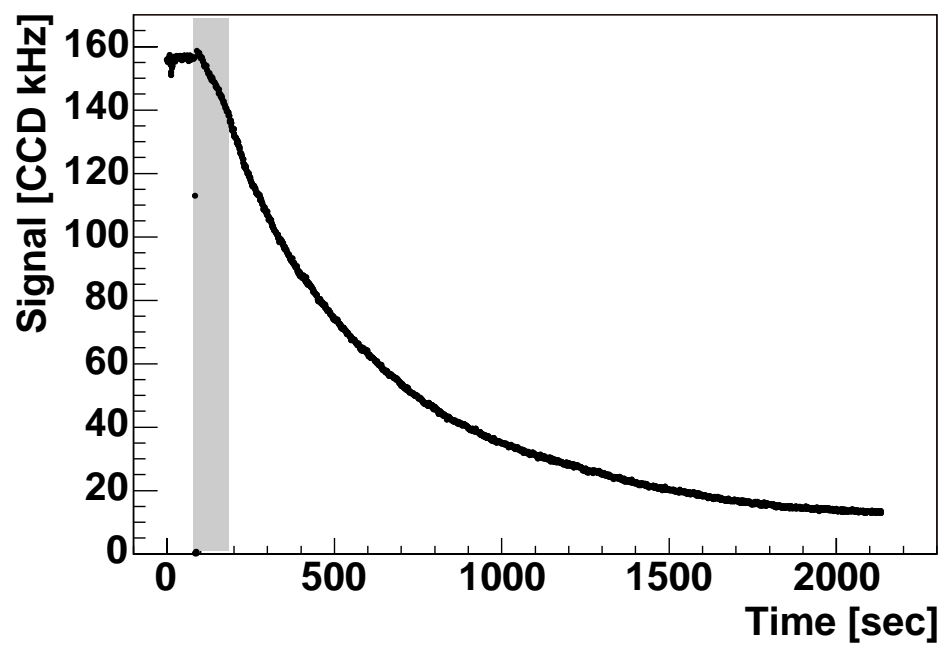

Figure 6.15: Xenon is introduced to a large cloud of barium ions in $1 \times 10^{-3}$ torr helium buffer. Beginning at $\mathrm{t}=80 \mathrm{sec}$, xenon leaks into the system for $100 \mathrm{~s}$, marked by the grey box. After the addition of $8.5 \times 10^{-4}$ torr xenon, the signal fades exponentially with $\tau=491$ s.

known as a function of gas manifold pressure and valve position. This allows us to add small pressures of trace gases when the vacuum system already contains high pressure helium. In Fig. 6.15, the grey box indicates a 100-second xenon fill, with a final xenon pressure of $8.5 \times 10^{-4}$. After the xenon is added to the system, the ion signal fades exponentially with $\tau=491 \pm 1 \mathrm{~s}$, where the exponential fit has $\chi^{2} / N D F=999 / 1238$. Once the signal has faded away, it does not return under any modification of the system pressure, laser frequency, or RF parameters.

Before discussing the possible explanations for the signal disappearance, we present a summary of the applicable data.

\subsubsection{Krypton unloading}

Ion cloud unloading occurs with krypton buffer gas as well as with xenon. Fig. 6.16 shows the response of ions trapped in UHV to krypton. For the first 50 seconds, the signal is constant at $5.25 \mathrm{kHz}$. At $\mathrm{t}=50$ seconds, the leak valve to the gas manifold is opened and krypton is let into the system in discrete steps, reaching an ultimate 


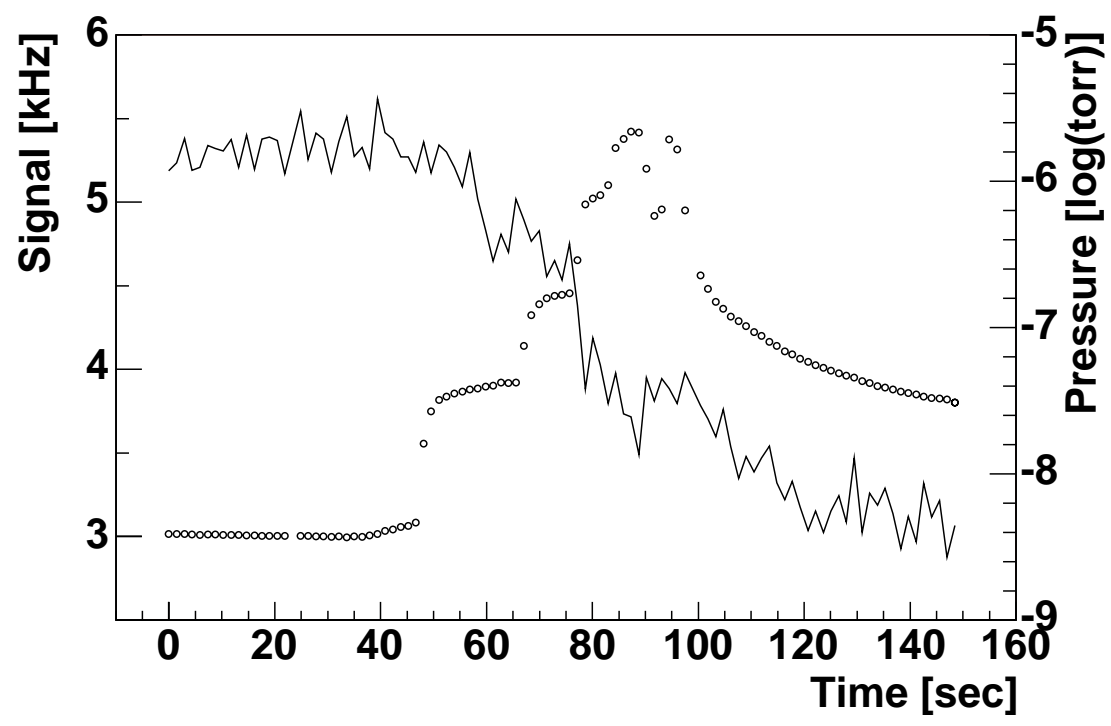

Figure 6.16: The signal (solid line) of a small cloud of ions fades as the krypton pressure increases (o). Krypton always unloaded the ion cloud.

pressure of $3 \times 10^{-6}$ torr. The ion pump is on for these measurements and evacuates the system when the leak valve is closed at $t=100$ seconds, however the pressure is never high enough to ion pump load. As soon as the krypton is introduced, the signal begins to fade until there are no ions left in the trap. All of the experiments with krypton, in a large variety of situations, demonstrate trap unloading with dynamics similar to xenon.

\subsubsection{Pressure dependence}

In order to measure the unloading time constant as a function of xenon pressure, we introduce the "step-unload" technique in which we measure multiple time-constants with a single ion cloud. The step-unload technique determines the impact of small partial pressures of xenon in high pressure helium buffer. Starting with a large ion cloud in a helium or argon buffer, we open the leak valve for a fixed time and bleed-in xenon gas. Then we shut the valve, monitor the signal decay time constant, and repeat until the cloud has disappeared. Fig. 6.17 shows a time series of the ion signal measured with this technique. The ion cloud is loaded in $1 \times 10^{-3}$ torr of helium and 


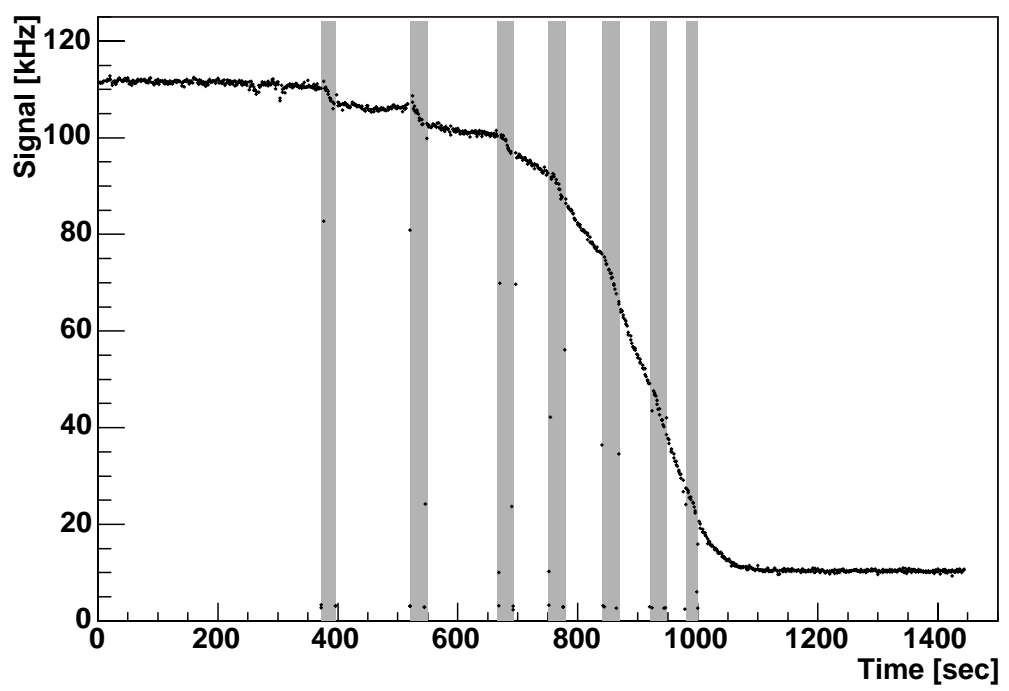

Figure 6.17: A large ion cloud in $1 \times 10^{-3}$ torr helium buffer gas. After $\sim 400$ seconds, $1.5 \times 10^{-4}$ torr of xenon is added to the system, denoted by the grey box. The signal decay is measured for $\sim 100$ seconds before xenon is again added to the system.

each xenon loading step, denoted by the grey boxes, adds $1.5 \times 10^{-4}$ torr of xenon gas to the system. The fade rate is measured for $100 \mathrm{~s}$ before adding more xenon. As the decay rate increases, the time in between xenon fills is decreased accordingly.

Fig. 6.17 contains many features worth of discussion:

1. The signal is very stable before xenon is added, fading at $4 \mathrm{~Hz} / \mathrm{s}$.

2. Each xenon fill causes an immediate dip in the signal level.

3. Each xenon fill increases in the fading rate.

The step-unload technique allows us to add small quantities of xenon to the vacuum system while the system is filled with a much higher pressure of buffer gas. Unfortunately, because we do not measure the partial pressure of xenon directly, the partial pressure of xenon added at each step is known only to $\pm 10 \%$. The leak valve introduces a systematic error since the leak rate is not constant over many operations of the valve. As the valve is actuated, the valve seat inelastically deforms, gradually increasing the flow rate through the valve. This systematic error makes an absolute 
determination of the step difficult, and also means we cannot compare data across many step-unload runs as the valve is actuated so much as to make the comparison meaningless. However, within a single step-unload run or for consecutive runs, the leak valve calibration is within the $10 \%$ error of each gas fill and so the gas fill systematic can be neglected.

The effect of increasing xenon pressure on the signal decay time constant can be seen clearly in Fig. 6.18, a summary of three xenon step-unload runs in helium buffer gas. The runs were taken with $1 \times 10^{-4}, 1 \times 10^{-3}$, and $1 \times 10^{-2}$ torr of helium buffer gas. An exponential decay is fit to each step of the step-unload series and the time constants are plotted as a function of the xenon pressure. At all helium pressures, the signal decay time constant depends exponentially on the xenon pressure. A single exponential is fit to the time constant vs. pressure curve with coefficients of $10,000 \mathrm{sec} / \operatorname{torr}_{X e}, 3,000 \mathrm{sec} / \operatorname{torr}_{X e}$, and $940 \mathrm{sec} / \operatorname{torr}_{X e}$ for the runs with $1 \times 10^{-2}$, $1 \times 10^{-3}$, and $1 \times 10^{-4}$ torr of helium buffer, respectively. Two major features of the data in Fig. 6.18 deserve notice: A) over a factor of 10 in xenon pressure and a factor of 100 in time constant, the time constant is an exponential function of xenon pressure; and B) the difference between $10^{-3}$ and $10^{-2}$ torr of helium buffer is a factor of 500 in the exponential decay rate with $10^{-3}$ torr partial pressure of xenon, and a change in the slope of the xenon pressure dependence.

\subsubsection{RF dependence}

In a variation of the step-unload technique, we measure the signal decay rate as a function of RF voltage. In this experiment, a large ion cloud was loaded in $1 \times 10^{-3}$ torr helium buffer. The signal decay time was $\geq 3 \times 10^{4}$ seconds before we added $3.3 \times 10^{-4}$ torr xenon gas. We measured the decay time constant for 5 settings of the RF voltage, keeping all other factors constant. Fig. 6.19 shows decay time constants as a function of RF voltage. Note that, unlike the previous plot, these data are plotted on a linear scale.

The data hints at a quadratic or exponential dependence of the time constant on the RF voltage, however the RF drive circuit could not sustain the high voltages 


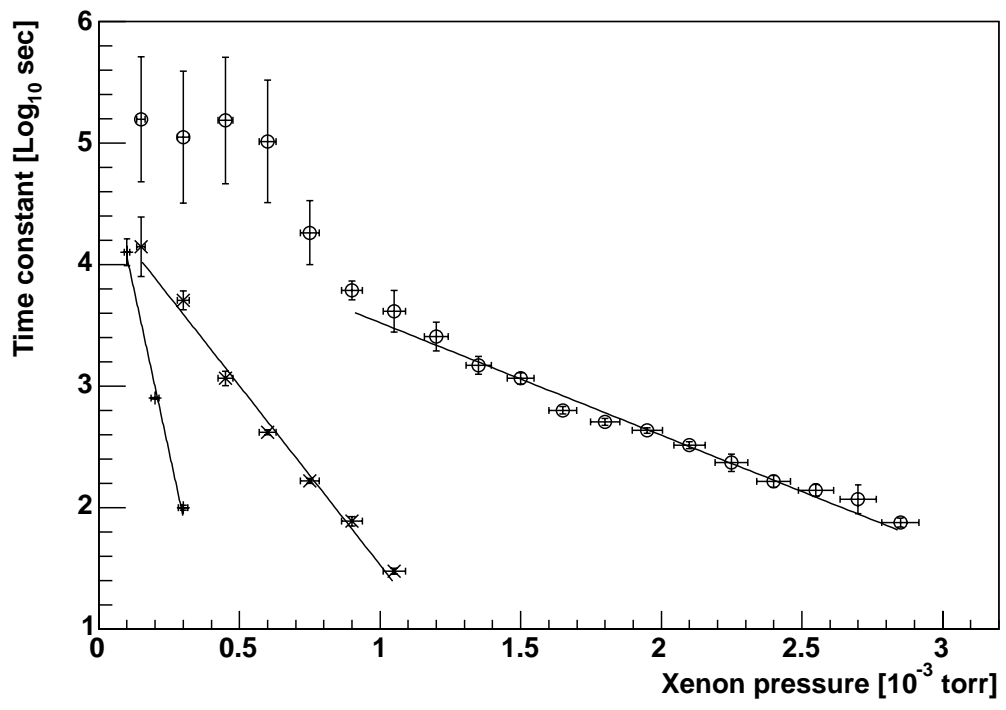

Figure 6.18: The time constants from three consecutive step-unload runs with $1 \times 10^{-2}$ (o), $1 \times 10^{-3}(\times)$, and $1 \times 10^{-4}(+)$ torr helium buffer plotted against xenon pressure. The solid lines are exponential fits of the decay time constant vs. xenon pressure with time constants of $940 \mathrm{sec} /$ torr $_{X e}$ for $1 \times 10^{-2}$ torr helium, $3000 \mathrm{sec} /$ torr $_{X e}$ for $1 \times 10^{-3}$ torr helium, and $10,000 \mathrm{sec} /$ torr $_{X e}$ for $1 \times 10^{-4}$ torr helium. Note the $\log$ scale. The points with $\tau>1 \times 10^{4} \mathrm{~s}$ were not included in the fits due to their large error. 


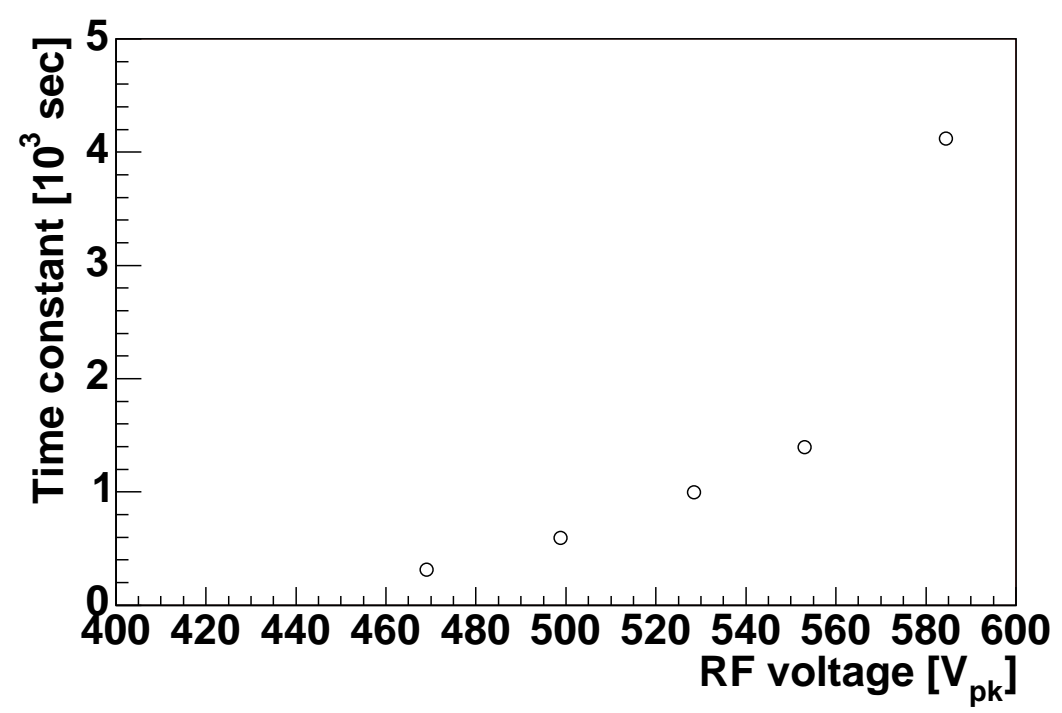

Figure 6.19: The decay time constants as a function of RF voltage. The ion cloud is trapped in $1 \times 10^{-3}$ torr helium buffer and $3.3 \times 10^{-4}$ torr xenon gas.

required in order to add measurements at high RF. Nonetheless, this hint gives a tantalizing hope that the signal time constant could be made effectively infinite merely by increasing the RF voltage. It should be noted however, that the partial pressure of xenon in this measurement was quite low and the trap will break down at substantially higher voltages, especially if the gas pressures of either xenon or helium gas are increased.

\subsubsection{Ion pump filter}

We tested the impact of the ion pump as a gas filter by adding a small $8 \mathrm{l} / \mathrm{s}$ ion pump to the system in line with the incoming gas flow but without a line of sight to trapping volume. After confirming that the small ion pump does not load the ion trap at high pressures, we measured the signal decay in xenon gas with the small ion pump running and found the signal decayed as though no vacuum pumps were operating. Although the $8 \mathrm{l} / \mathrm{s}$ is not the same style of ion pump as the $20 \mathrm{l} / \mathrm{s}$ pump with which signals were measured in high pressure xenon, it is unlikely that any gas impurity was removed by the $20 \mathrm{l} / \mathrm{s}$ pump that was not removed by the $8 \mathrm{l} / \mathrm{s}$ pump. 
In a similar vein, we use the $20 \mathrm{l} / \mathrm{s}$ pump as a filter by generating a large signal in $1 \times 10^{-3}$ torr xenon gas with the ion pump on. Then the gas valve is closed before the ion pump is turned off, allowing the ion pump to fully clean any impurities that could be present in the closed vacuum system. Several seconds after the gas valve is closed, the ion pump is turned off and the signal disappears immediately. Consequently, if the ion pump influences the ion signal by differentially pumping an impurity (instead of by ion pump loading as mentioned before), it must be an impurity generated within the vacuum system due to the presence of the krypton or xenon.

\subsection{Unloading mechanism}

The fact that the signal decay rate depends on the helium pressure eliminates many possible explanations of the decay's origins. One remaining plausible explanation is that the unloading process has a reversible intermediate state which helium can break apart. One such state is a weakly bound, charged molecule such as barium xenide $\left(\mathrm{BaXe}^{+}\right)$. In this model, a barium ion forms a molecule with xenon which is too heavy to stay trapped. A short time after its creation, the $\mathrm{BaXe}^{+}$leaves the trap unless it collides with another gas atom and breaks apart. This model qualitatively predicts the helium pressure dependence and the RF voltage dependence: the higher the helium pressure, the more likely a collision occurs before the molecule leaves the trap; the lower the RF voltage, the shallower the trap and the less time required before the molecule unloads. However, the assumption that $\mathrm{BaXe}^{+}$is the intermediate state fails to explain the entire phenomenology because krypton also causes the signal to decay. The likelihood that Ba-II can form a molecule with xenon in low pressure gas is quite small since a three body collision is required for formation. Moreover, a single collision is expected break the weakly bound molecule. The probability that Ba-II forms a molecule with krypton is even lower.

Assuming the ions do not form molecules directly with xenon and argon, additional complications are required to model the observed phenomenology. First, as discussed in $\S 6.6 .4$, the signal fades in xenon after the fill valve is closed and the ion pump has purified the gas in the vacuum tank. Consequently, xenon must generate 
the molecule-forming impurity from within the vacuum system, either from adsorbed molecules on the vacuum walls or from within the ion pump. Second, the combined impurity formation and intermediate state formation must exhibit the exponential pressure dependence observed in Fig. 6.14 and Fig. 6.15. Naively, the xenon continuously generates the molecule-forming impurity inside the vacuum system, causing a continuous increase of the ion-signal decay rate instead of the observed constant rate. Furthermore, as shown in Fig. 6.18, the decay time constant shows an exponential dependence on the xenon pressure, while impurity formation has a linear pressure dependence. To account for the observed behaviours, the detailed dynamics must be quite complicated. Finally, in vacuum the trap stably contains ions from $\mathrm{m}=35$ to $\mathrm{m}=280$ and in helium, a much larger mass range. Thus, the molecule cannot unload from the trap unless it is very heavy. However, even if the molecule does not unload, the barium ion will no longer fluoresce and the trap will appear empty. To test the dark molecule hypothesis, we cycle the pressure in the vacuum tank, first adding xenon and waiting for the signal to fade, then evacuating the system and adding helium. If molecules remain trapped, then the added helium will break apart the molecule and the Ba-II fluorescence should return. Since we find no signal return, we conclude that the ions permanently leave the trap when the signal decays, complicating the molecular origin for the signal fade. In short, although we cannot absolutely rule out molecular formation, the mechanism would have to be quite complicated in order to explain all of the available data.

Fortunately, a much simpler mechanism well describes the observed phenomenology: Diffusion unloading. As discussed in $§ 5.5$, a trapped ion which collides with a neutral gas atom can take energy from the RF electric field. The combination of many such random collisions leads to a random walk of the maximum radius similar to diffusion. The extent of the diffusion depends on the size of each individual step, and consequently, on the mass of the colliding gas atom. The resulting spatial distribution explains not only the data in $\S 6.4 .2$, but also the unloading observed with krypton and xenon. Collisions with krypton and xenon cause a large change in the radius of the trapped ion orbit while the lighter helium and argon do not. After several consecutive collisions, each of which causes an increase in the orbit, the ion hits 
an electrode and unloads. The addition of helium to the system damps ion motion in between collisions with heavy atoms, decreasing the probability that the ions unload and thereby increasing the trapping time. Unlike gas impurities or molecule formation, diffusion unloading qualitatively explains all of the observed phenomenology without the addition of ad-hoc assumptions. 


\section{Chapter 7}

\section{Diffusion unloading simulation}

In order to test the diffusion unloading hypothesis, we developed a simple Monte Carlo model of an ion trapped in buffer gas. The program includes two buffer gases in order to effectively model the step-unload data presented earlier. However, the simulation does not include the long-range ion-ion coulomb interaction, and so must not be considered a complete description of the experimental data. Nonetheless, the simulation provides a framework with which to evaluate the effect of collisions on the trapping dynamics.

\subsection{Simulation configuration}

The simulation solves the differential equations of motion for a barium ion trapped in an ideal RF trap taking the RF parameters of the trap operation as input. The simulation uses an adaptive, fourth-order Runge Kutta numeric integration algorithm [82] to propagate the trapped ion in time, taking the fewest steps possible to achieve a specified accuracy. With the chosen accuracy, the difference between numeric integration and the analytic solution is less than 1 percent for a $10 \mathrm{~s}$ integration.

The program outputs the envelope of a trapped ion positions as a function of time. The ion motion is calculated in $20 \mathrm{~ns}$ microsteps, with the largest radius microstep in a $10 \mu$ s interval recorded as the envelope data. This technique averages over the fast time scales of the macro- and micro-motion, reducing the amount of storage required 
while recording the physically significant data. In effect, the envelope technique records the amplitude, $A$, of the Mathieu equations of motion described in $§ 5.5$. Under ultra-high vacuum conditions, the simulated trap parameters match the real trap parameters listed in Table 4.1.

Helium and buffer gas collisions are implemented using the Langevin model, following the discussion in $§ 5.4 .2$ and $§ 5.5$. At every microstep, the time intervals until the next envelope step, helium collision, and buffer gas collision are calculated, and the ion motion integrated for the shortest of the three intervals. The envelope interval is fixed, while the helium and buffer gas collision intervals are chosen from exponential distributions with appropriate time constants. Each collision randomizes the velocity of the ion in the center of mass frame, correctly accounting for the mass of the gas scatterer as described in $§ 5.4 .1$. The buffer gas atom has a random velocity chosen from a Maxwell-Boltzmann distribution with mean temperature of $300 \mathrm{~K}$.

This brute force approach avoids the problems associated with approximation techniques at the price of long run times. On a 1.6 GHz AMD Athlon processor, the simulation takes $\sim 1,000$ real-time seconds to calculate each simulation-time second. This ratio prohibits us from directly simulating the long decay times found in the actual experiment. Nonetheless, the simulation provides valuable insight into the unloading mechanism, confirming that diffusive unloading is the primary cause of the ion signal decay in massive buffer gases.

\subsection{Comparison to theory}

Before calculating diffusion unloading for barium in xenon, we compare the results of the simulation to the theoretical expectations of Dehmelt's theory, discussed in $§ 5.1$. The theory makes two testable predictions: A) a buffer gas much lighter than the trapped ion cools the ion; and B) a buffer gas much heavier than the ion heats the ion. First, we confirm that helium cools the motion of hot ions by calculating the envelope of an ion inserted into the trap at $r=1 \mathrm{~cm}$, at which point the ion has a potential energy (see Eq. 4.18) of $2.7 \times 10^{6} \mathrm{~K}$. The mean time between collisions is $1 \times 10^{-5} \mathrm{~s}$, equivalent to $100 \mathrm{mTorr}$ of helium (by Table 5.2). The simulated ion radius is fit to 


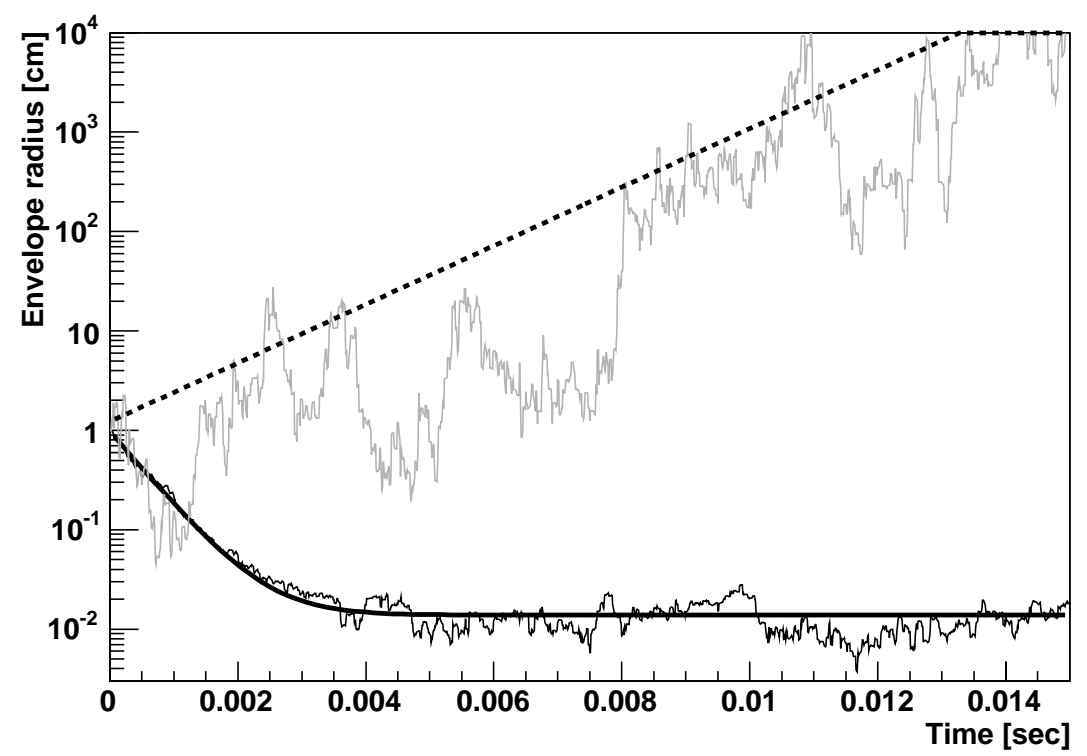

Figure 7.1: Simulation of trapped barium ion behaviour in buffer gas as a function of buffer gas mass. In the case of helium $(\mathrm{m}=4$, black, solid line), the initial large radius is cooled. For the much heavier gas radon $(\mathrm{m}=222$, dashed, grey line), the ion radius increases exponentially.

an exponential decay plus a constant. As shown by the solid black line in Fig. 7.1, collisions with helium damp the maximum radius from $1 \mathrm{~cm}$ to $0.0138 \mathrm{~cm}$ with a time constant of $\tau=.58 \mathrm{~ms}$. The ion reaches equilibrium at $.0138 \mathrm{~cm}$, undergoing small excursions due to collisions about a constant radius. The pseudo-potential depth at $0.0138 \mathrm{~cm}$ is $348 \mathrm{~K}$, indicating that the ion thermalizes at a temperature slightly above the buffer gas temperature of $300 \mathrm{~K}$.

The dashed grey line in Fig. 7.1 is the envelope radius for barium collisions with radon $(\mathrm{m}=222)$. As Dehmelt suggests, collisions with a buffer gas heavier than the trapped ion cause exponential growth in the ion radius, and consequently the ion radius is fit to an increasing exponential. With radon as the background gas, the radius never reaches a steady state value, instead increasing with a time constant of $680 \mathrm{~cm} / \mathrm{s}$. 


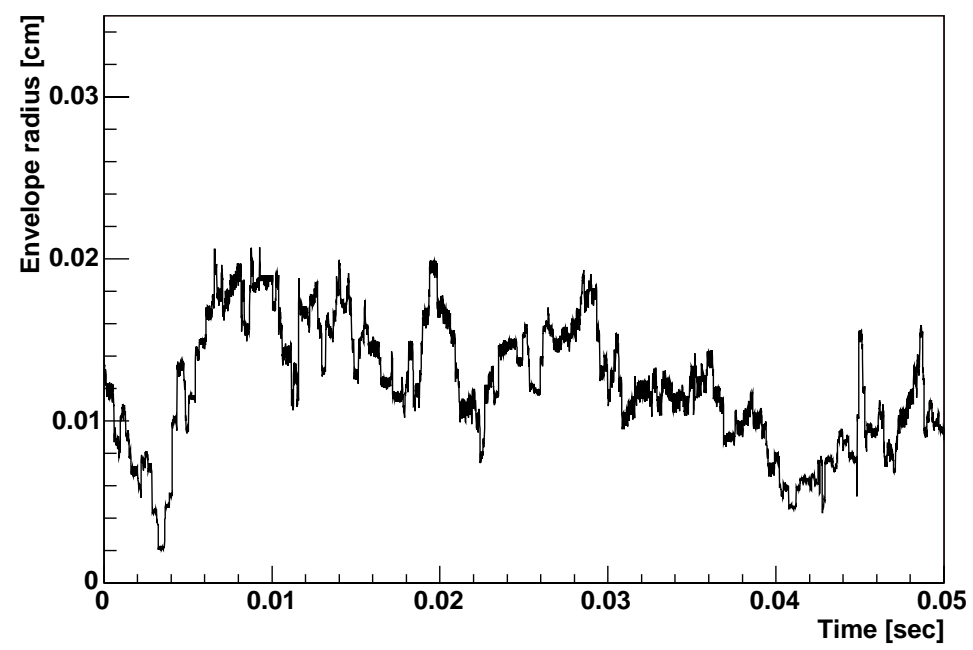

Figure 7.2: The simulated time series of a barium ion trapped in helium buffer gas. The mean time between collisions is $100 \mu \mathrm{s}$, the envelope size is $10 \mu \mathrm{s}$, and the total run time is $50 \mathrm{~ms}$.

\subsection{Trapped ion diffusion}

The motion of the ion in Fig. 7.1 deserves special notice, since the inherent randomness of the motion causes diffusive unloading. Fig. 7.2 shows the envelope of an ion randomly colliding with helium once every $100 \mu \mathrm{s}$. The time series covers $50 \mathrm{~ms}$ during which there are 535 collisions. The ion behaviour is qualitatively similar to Brownian motion; many small, random events generate the macroscopic, well defined average radius and RMS.

Eq. 5.25 describes the "random walk" nature of the colliding trapped ion as $\partial A_{\max }= \pm A /\left(1+m_{I} / m_{B G}\right)$, where $A$ is the maximum radius of the ion, and $m_{B G}$ and $m_{I}$ are the mass of the background gas and the ion, respectively. As the background gas mass increases, the random walk stepsize increases, causing a corresponding increase in the average radius and rms. Compared to helium, collisions with xenon cause larger random fluctuations, as shown in the time series with xenon buffer gas in Fig. 7.3., The larger fluctuations cause a corresponding increase in the average radius and the RMS.

In keeping with the Brownian motion analogy, we histogram the value of the x-axis 


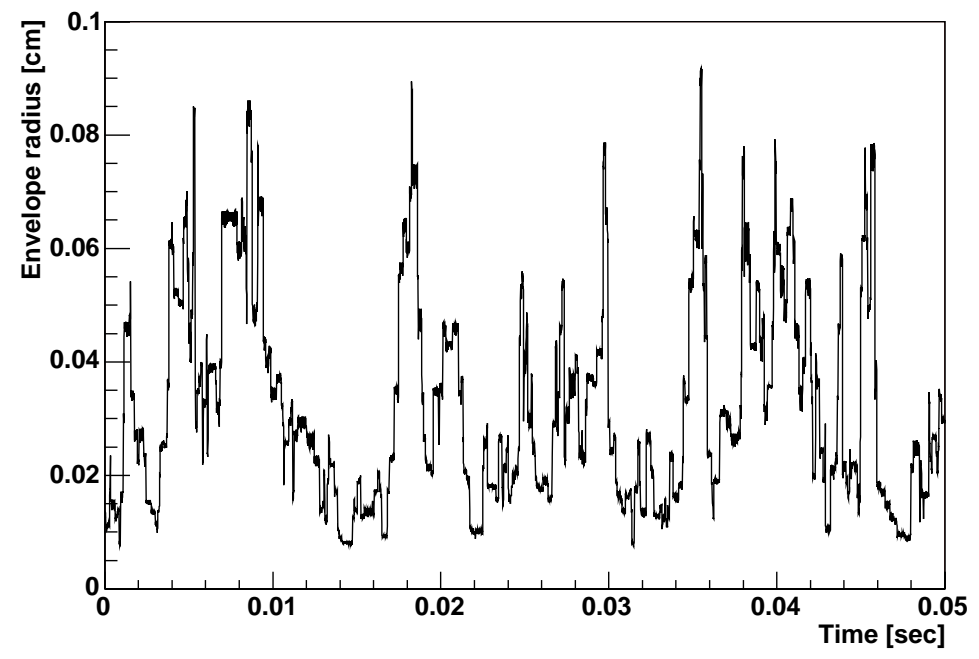

Figure 7.3: The simulated time series of a barium ion trapped in xenon buffer gas. The mean time between collisions is $100 \mu \mathrm{s}$, the envelope size is $10 \mu \mathrm{s}$, and the total run time is $50 \mathrm{~ms}$.

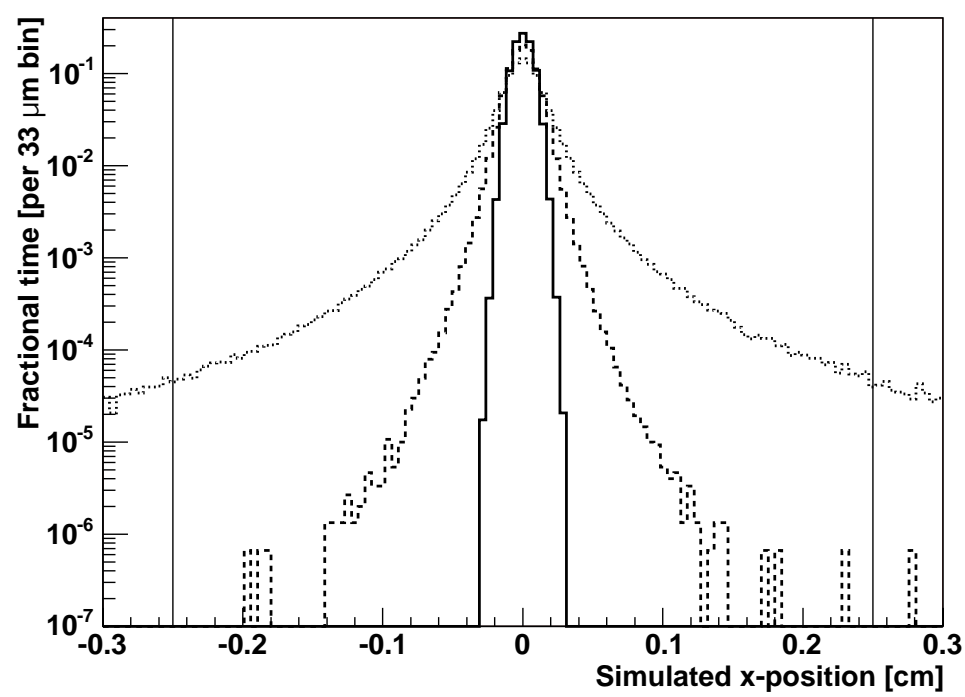

Figure 7.4: Histograms of the x-axis position of a barium ion trapped in helium (solid line), krypton (dashed line), and xenon (dotted line) buffer gases. The mean time between collisions is $10 \mu \mathrm{s}$, the envelope size is $20 \mu \mathrm{s}$, and the total run time is $5 \mathrm{~s}$. The lines at $\mathrm{r}= \pm 0.250 \mathrm{~cm}$ mark the trap ring electrode radius. 
position of the simulated ion in Fig. 7.4. These histograms allows us to investigate the behaviour of the macroscopic observable, the maximum ion radius. Each bin along the horizontal axis represents an envelope radius, while the vertical axis represents the fraction of time the ion spends at that radius. The three histograms summarize the data for helium, krypton and xenon. As expected from Fig. 7.2, the helium histogram has a well defined mean and small deviation; the ion orbit never extends beyond $\mathrm{r}=$ 0.04 within the time duration represented by the simulation. The krypton and xenon distributions, on the other hand, both extend to high radius due to the increased size of each collision. In fact, the krypton envelope extends to $.28 \mathrm{~cm}$ and the xenon envelope extends to $6 \mathrm{~cm}$ !

The radial extent of the ion distribution merits particular attention due to the finite trap size. The vertical lines in Fig. 7.4 at $x= \pm 0.250 \mathrm{~cm}$ demarcate the axial electrode spacing. In practice, the non-ideal RF electrode geometry reduces the effective trapping volume; $0.250 \mathrm{~cm}$ is an upper limit on the trapping radius. For

both krypton and xenon buffer gas, the simulated ion orbits extend beyond the trap electrodes. In the real, finite hyperbolic trap, these high radius orbits cause diffusion unloading.

\subsection{Simulated buffer gas spectra}

Several details of ion cloud behaviour in buffer gas must be explained by the simulation. In particular we simulate the observed RF independence of the velocity width and the observed signal increase with RF voltage, as in Fig. 6.12. We also calculate the velocity spectrum as a function of gas pressure in order to simulate the data as shown in Fig. 6.10.

\subsubsection{RF independent velocity distribution}

We simulate the velocity distribution along the laser axis as a function of the RF voltage. The voltage varies from $300 V_{p k}$ to $600 V_{p k}$ with the gas pressure held constant at 1 mTorr of helium. Fig. 7.5 shows the velocity distributions for 360, 480 


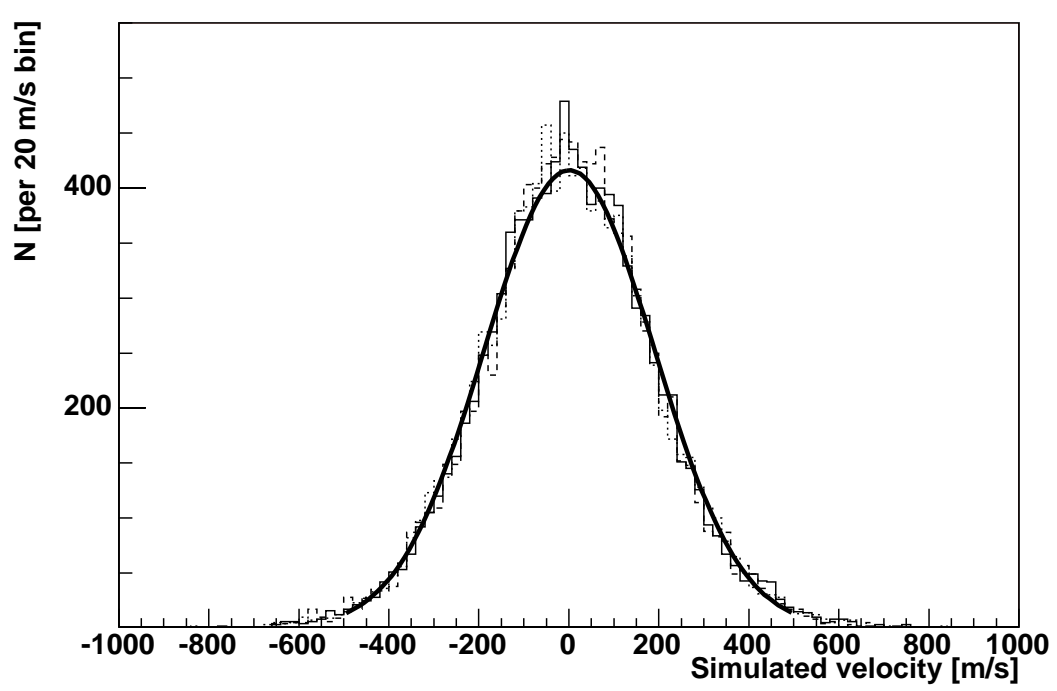

Figure 7.5: Three representative velocity distributions for $V_{p k}=360 \mathrm{~V}$ (dotted line), $480 \mathrm{~V}$ (solid line), and $600 \mathrm{~V}$ (dashed line). The gaussian fit to $V_{p k}=360 \mathrm{~V}$ has $\sigma_{V}=190 \mathrm{~m} / \mathrm{s}$. The distributions are nearly identical for each RF voltage.

and $600 V_{p k}$. The solid line is a fit to the $360 V_{p k}$ distribution with $\sigma_{V}=190 \mathrm{~m} / \mathrm{s}$. The 1/e width as function of RF voltage is shown in Fig. 7.6.

The $1 / e$ velocity of $\sim 190 \mathrm{~m} / \mathrm{s}$ is somewhat lower than the value of $\sim 240 \mathrm{~m} / \mathrm{s}$ measured in Fig. 6.12. Nonetheless, the $190 \mathrm{~m} / \mathrm{s}$ average velocity corresponds to an apparent thermal distribution of $590 \mathrm{~K}$, substantially higher than room temperature. Moreover, the distributions in Fig. 7.5 are basically independent of the RF voltage, in agreement with the data.

\subsubsection{RF dependent signal amplitude}

The fluorescence signal measured experimentally is proportional to the number of ions trapped and the fraction of time those ions spend in the laser beams. At the trap center, the laser beam has a diameter of $100 \mu \mathrm{m}$, so we model the relevant fluorescence volume as a $100 \mu \mathrm{m}$ sphere at the trap origin. Fig. 7.7 shows the radial distribution of the trapped ions for three RF voltages, spanning the parameters used in Fig. 6.12. 


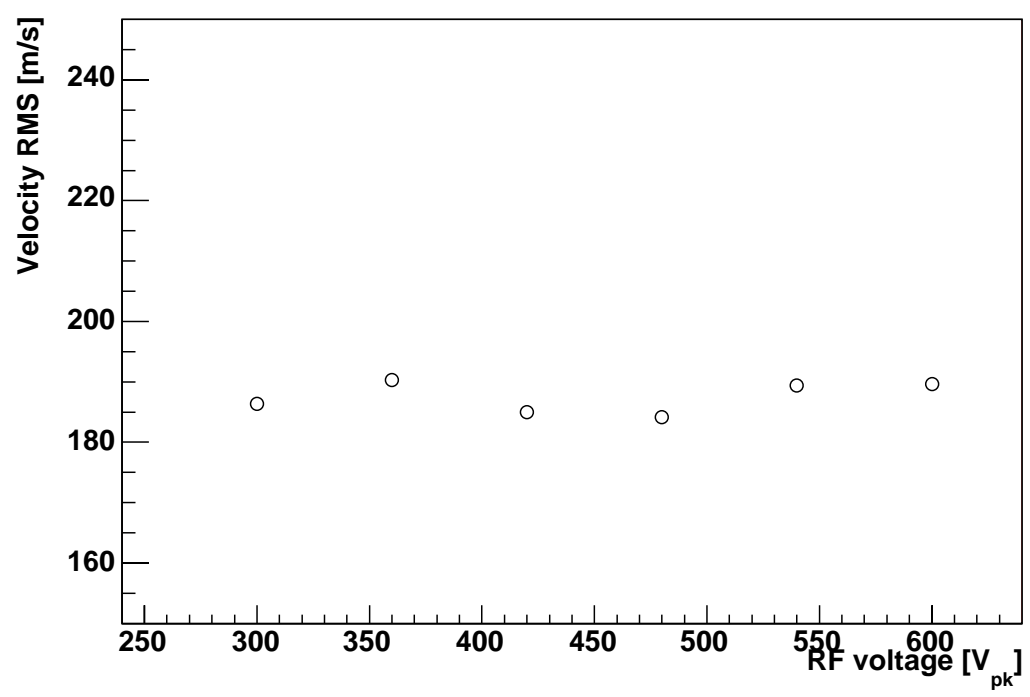

Figure 7.6: The velocity $1 / e$ width as a function of $\mathrm{RF}$ voltage. The average $1 / e$ value at all $R F$ voltages is $187 \mathrm{~m} / \mathrm{s}$.

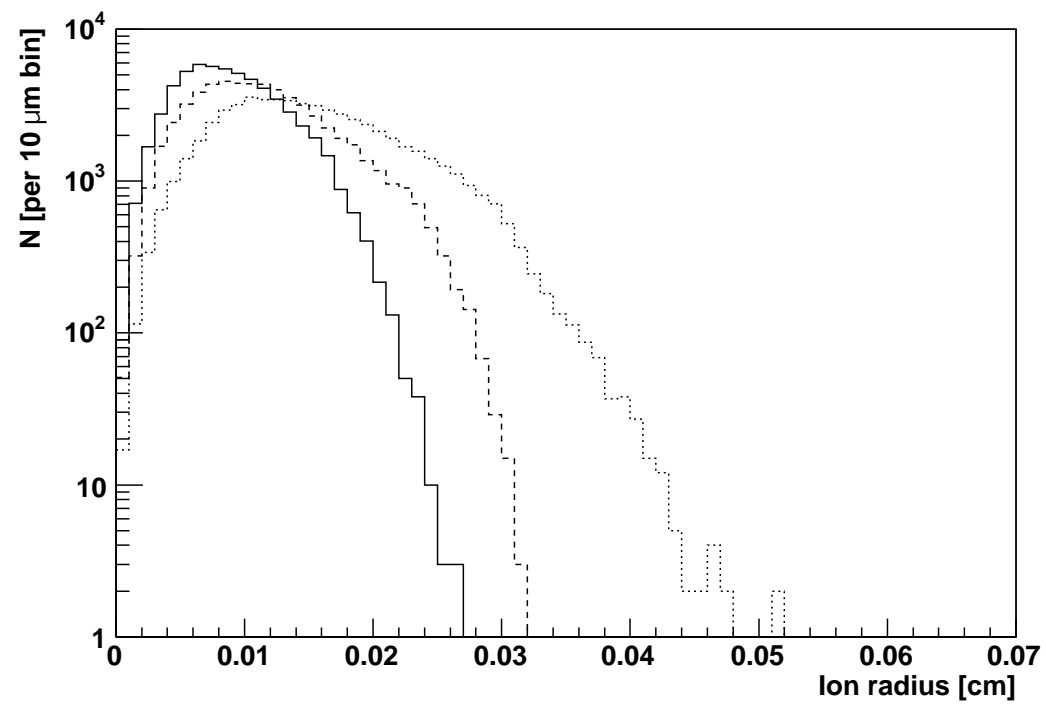

Figure 7.7: The ion radius as a function of RF voltage for a simulated trapped ion in 1 mTorr helium buffer. As the voltage increases from $360 V_{p k}$ (dotted line) to 480 $V_{p k}$ (dashed line) to $600 V_{p k}$ (solid line), the ion distribution concentrates in the trap center. 


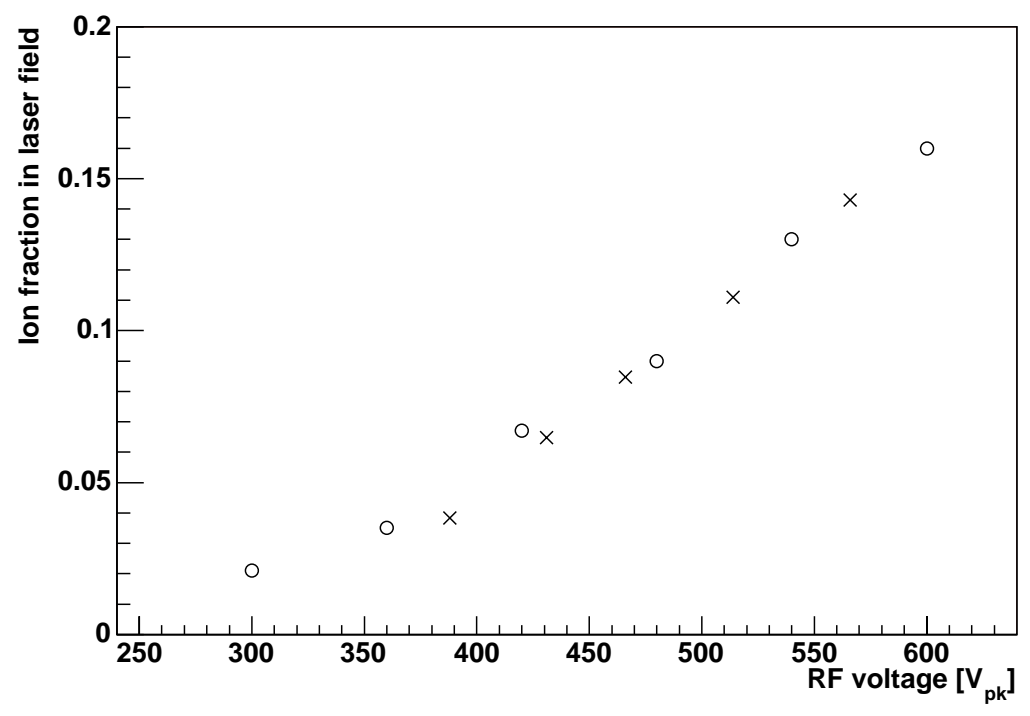

Figure 7.8: The simulated fraction of the ions inside a $100 \mu \mathrm{m}$ diameter sphere as a function of RF voltage (o) plotted with the data for barium ions in 1 mTorr helium $(x)$.

The fraction of the ion cloud within a $100 \mu \mathrm{m}$ diameter is plotted in Fig. 7.8 as a function of RF voltage. For comparison, the data from Fig. 6.12 is plotted on the same graph. The data is renormalized by a constant scaling factor chosen by setting the $V_{R F}=560 V_{p k}$ data point to the value calculated by interpolating the simulation results. The data and the simulation predict a similar linear dependence of the ion signal on $R F$ voltage, and both predict unloading around $V_{R F} \simeq 300 V_{p k}$. Specifically, when fit to a linear polynomial, the data suggests that the ion signal disappears at $V_{R F}=330 V_{p k}$ while the simulation predicts signal disappearance at $V_{R F}=290 V_{p k}$. Because of the renormalization required to compare the simulation to the data, only the slope retains physical significance. Nonetheless, the correspondence between the simulation and data is quite remarkable.

\subsubsection{Pressure dependent velocity spectra}

The spectra presented in $\$ 6.4 .3$ demonstrate a constant linewidth for an ion cloud trapped in helium gas ranging in pressure from $6.9 \times 10^{-5}$ torr to $6.2 \times 10^{-2}$ torr. 


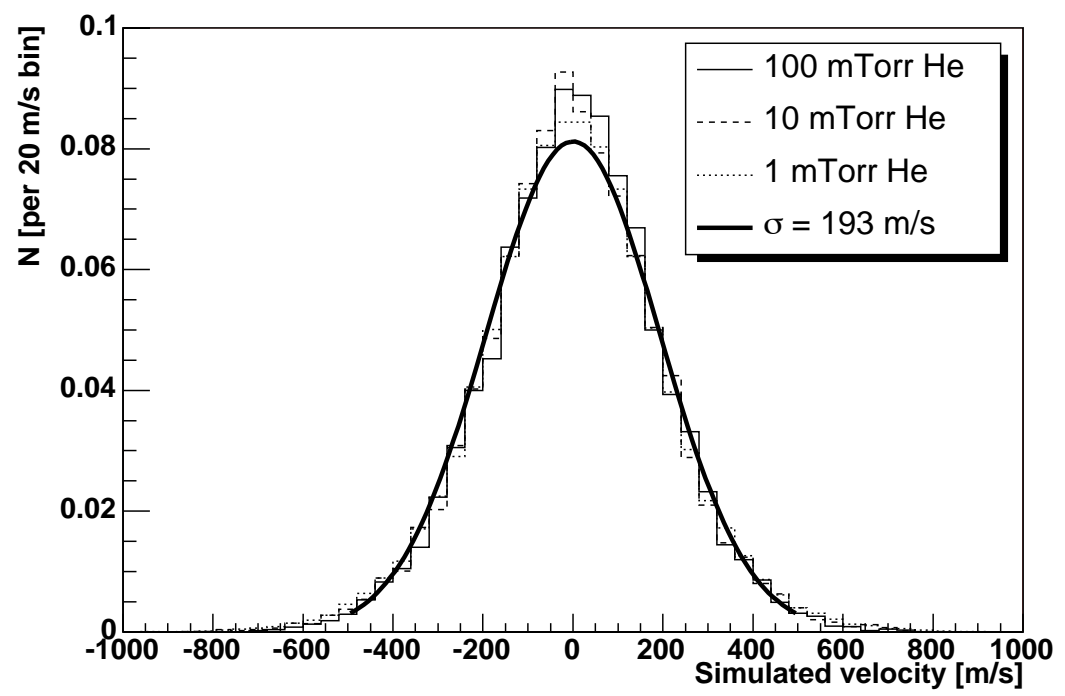

Figure 7.9: Simulated velocity spectra for ions trapped in helium gas at 100 (solid line), 10 (dashed line), and $1 \mathrm{mTorr}$ (dotted line). The $1 \mathrm{mTorr}$ simulation is fit to a gaussian with $\sigma_{v}=193 \mathrm{~m} / \mathrm{s}$. The simulated velocity distribution is constant as a function of pressure, in excellent agreement with experimental data.

The experimental spectra all have a line width of $\sigma_{V} \approx 240 \mathrm{~m} / \mathrm{s}$. We simulated the doppler line width as a function of helium pressure for $1 \times 10^{-5}, 1 \times 10^{-4}$, and $1 \times 10^{-3}$ torr of helium buffer gas. The results, plotted in Fig. 7.9 show a constant linewidth for all the pressures. The $1 \times 10^{-3}$ torr simulation is fit to a gaussian with a linewidth of $220 \mathrm{~m} / \mathrm{s}$, in good agreement with the experimental data.

The velocity spectra of the ions remains constant under all the conditions of voltage and pressure simulated here and observed in experiment. Although this result is somewhat counter-intuitive, it can be simply explained by considering the kinematics of the trapped ions, the effect of collisions, and the averaging effect of the measurements. During the course of a measurement, the ion experiences many collisions and hence undertakes many orbits, each with a different velocity along the laser axis. Each oscillating velocity causes a modulation in the absorption spectrum, as discussed in $\S 3.4$; the result of the collisions is to change the modulation depth. Averaged over many collisions, the observed velocity profile has a gaussian distribution. The effect of changing pressure changes the rate at which the collisions occur, but does not change 
the range of modulation depths that the ion experiences. Thus, the velocity profile is independent of pressure provided the observation averages over many collisions.

To explain the voltage independence of the velocity, we refer to the adiabatic approximation introduced in $\S 4.2$, for which the effective potential experienced by the ion is given by $\psi(\bar{z})=\frac{e}{4 m \Omega^{2}} E_{\Omega}^{2}$. The average velocity of an ion in the pseudo-potential approximation is given by $\bar{v}=z_{T} \times \omega_{z}$, where $z_{T}$ is the average ion displacement at a temperature $T$, and $\omega_{z} \propto V_{A C}$ is the oscillation voltage. In thermal equilibrium, the pseudo-potential energy is equal to the thermal energy,

$$
\psi(z)=\frac{e}{4 m \Omega^{2}} \frac{V_{A C}^{2} z_{T}^{2}}{z_{0}^{2}}=k T,
$$

from which we extract the average ion radius in thermal equilibrium,

$$
z_{T}=\sqrt{k T \frac{z_{0}^{2}}{V_{A C}^{2}} \frac{4 m \Omega^{2}}{e}} \propto \frac{1}{V_{A C}} .
$$

As the $\mathrm{RF}$ voltage to the trap increases, the average radius decreases while the average frequency of the ion motion increases. Taken together, the velocity, $\bar{v}=z_{T} \times \omega_{z}$, remains constant and the velocity distribution has no dependence on the RF voltage.

\subsection{Simulated decay time constant}

Instead of plotting the simulated radial distribution, we histogram the intervals between high radius excursions. Fig. 7.10 shows the time intervals between unloadings for a $5 \mathrm{~s}$ simulation of barium in xenon with an average time between collisions of 10 $\mu$ s (equivalent to 22 mTorr). The signal fades with an exponential decay time constant of $5.2 \mathrm{~ms}$. For practical reasons, these simulation results cannot be compared directly to data: we cannot observe $5 \mathrm{~ms}$ decay times in the laboratory, nor can we simulate the observed 10 - 1000 second decays with reasonable simulation durations.

Instead of directly reproducing the data using the simulation, we simulate the unloading time constant dependence on xenon and helium pressures, similar to the 


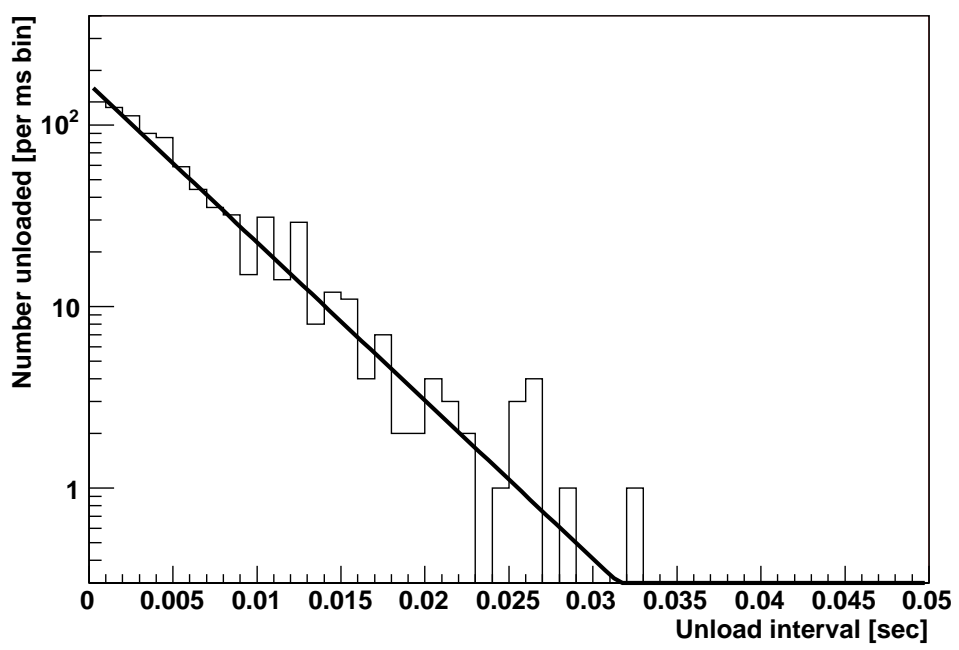

Figure 7.10: Interval between unloadings for a barium ion in xenon buffer. The mean time between collisions is $10 \mu \mathrm{s}$, and the ion orbit is simulated for $5 \mathrm{~s}$ during which there are 870 unloads with an average interval of $5.2 \mathrm{~ms}$.

data in Fig. 6.18. For a fixed helium collision rate, the exponential unloading time constant is calculated for a range of xenon collisions rates. The results are plotted on a log-log plot of decay time versus xenon partial pressure in Fig. 7.11. Single sided arrows indicate the lower limit of the time constant for the simulations with no unloading events. The lower limits are calculated for $95 \%$ confidence level using the Feldman-Cousins technique described in [83].

Recall from Table 5.2 that the collision cross section for an ion with xenon is 5 times larger than the cross section for an ion with helium. For the 1 mTorr helium simulation, the ion collides with xenon more frequently than with helium for all of the xenon pressures simulated in Fig. 7.11. As a result, the simulated decay time depends only on the xenon pressure, and the data are fit by an inverse pressure dependence,

$$
\tau(P)=(0.129 \pm .003) P^{-1.0} \quad[s] .
$$

The $P^{-1}$ scaling of the time constant arises because the unloading rate depends on the number of collisions; an ion unloads, on average, after $N_{\text {unload }}$ collisions. If the 


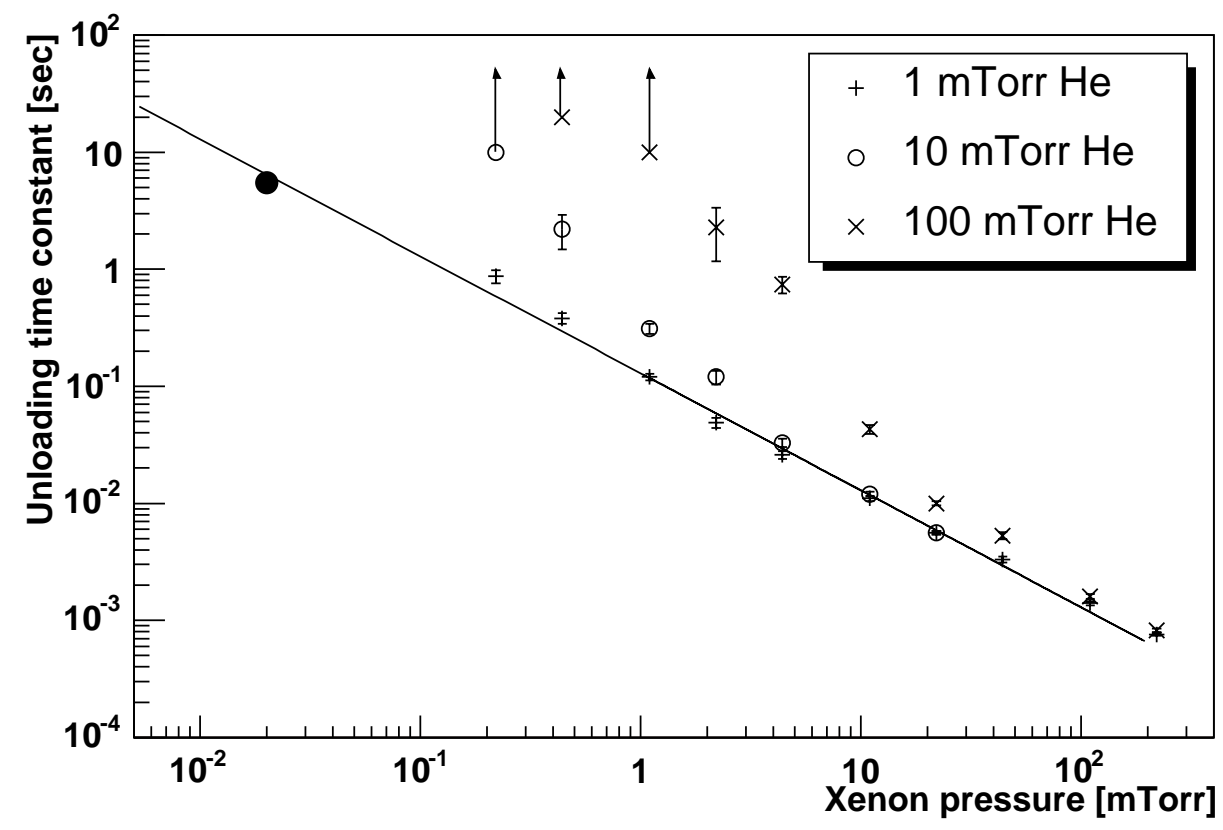

Figure 7.11: Simulated pressure dependence of the unloading time constant as a function of xenon pressure with 1 mTorr helium (+), 10 mTorr helium (o), and 100 mTorr helium $(\times)$. No unloading events were observed for the simulated data points marked with single arrows; the points are $95 \%$ lower limits. The $1 \mathrm{~m}$ Torr data is fit to $\tau=0.13 \times P^{-1}$ (solid line). The experimentally measured decay shown in Fig. 6.14 is plotted on the same graph for comparison (filled circle). 
pressure is high, then the ion experience more collisions per second and the unloading rate is faster, and vice versa.

When the ion collides with helium more frequently than it collides with xenon, the pressure dependence deviates from Eq. 7.3. For 10 mTorr of helium buffer, the ion collides more frequently with helium than with xenon when the xenon pressure is below 2 mTorr. Below $2 \mathrm{mTorr}$, the decay time constant is much larger than predicted by the $P^{-1}$ scaling. Similarly, the 100 mTorr helium buffer simulation deviates for xenon pressures below $\sim 20$ mTorr. Nonetheless, all three simulations tend to the same xenon pressure dependence when collisions with xenon predominate. When the trapped ion dynamics are dominated by helium, a different pressure scaling applies. Unfortunately, the time constants involved for high helium pressure simulations are so long that we can only place lower limits on the time constants.

We can compare the simulation in Fig. 7.11 with the data in Fig. 6.14 which shows a $5.5 \mathrm{~s}$ signal decay for $2.0 \pm .1 \times 10^{-5}$ torr xenon in $1 \times 10^{-6}$ torr helium. For this data point, the xenon pressure is substantially higher than helium so the $P^{-1}$ decay rate dependence should hold. Extrapolating the simulated decay rates to $2.0 \times 10^{-5}$ torr gives $6.45 \pm .36 \mathrm{~s}$, a slightly larger decay time than observed experimentally. The error on the calculated decay rate primarily results from uncertainty in the xenon pressure when the data in Fig. 6.14 was taken. The agreement between the simulation and experimental result is shown in Fig. 7.11, which includes the data (solid circle) and the simulated $P^{-1}$ dependence (solid line).

The simulation disagrees with the data involving large ion clouds presented in Fig. 6.18. For $10 \mathrm{~m}$ Torr of helium gas and $1 \mathrm{mTorr}$ of xenon gas, according to the data the ion signal fades with $\tau>10^{3} \mathrm{~s}$, while the simulation suggests the signal should fade with a time constant of $\tau \sim 1 \mathrm{~s}$. We believe the difference arises from the fact that the simulation neglects the long range contributions of the ion-ion coulombic potential and of the long range induced dipole potential. With its infinite range, the coulomb collision cross-section is much larger than the cross section for collisions between ion and neutrals. Consequently, the ion dynamics in the large clouds used in Fig. 6.18 are dominated by the ions themselves, with ion-ion collisions damping out the large amplitude, high velocity motion required for unloading. Ion-ion interactions might 


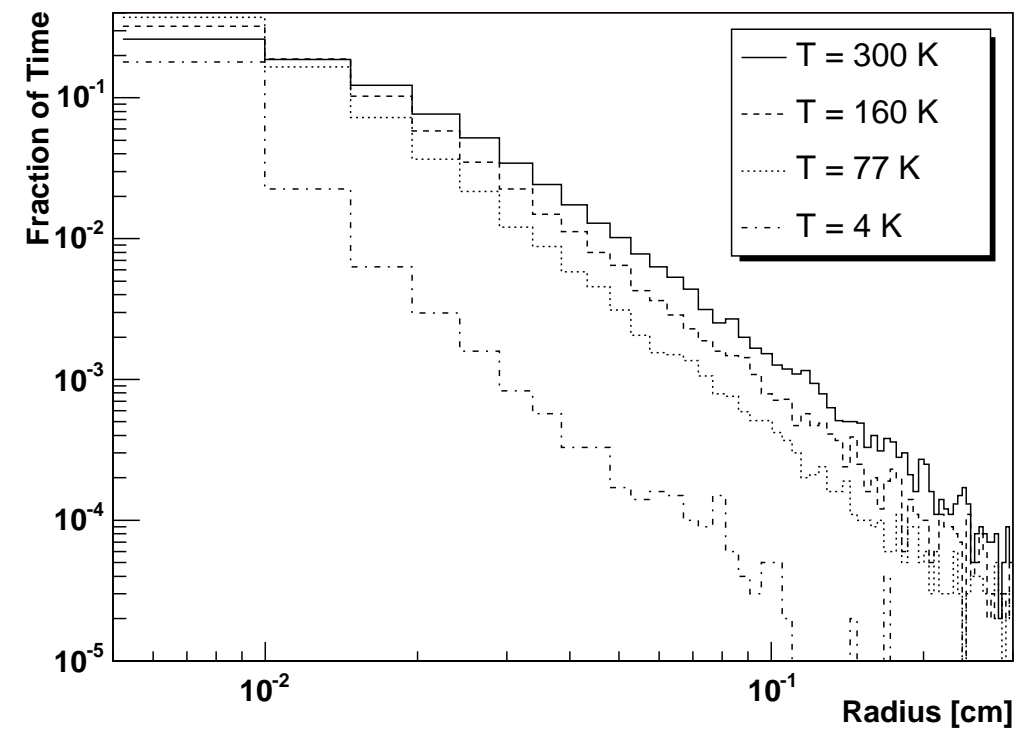

Figure 7.12: The spatial distribution for ions trapped in 22 mTorr of xenon gas at 300 $\mathrm{K}$ (solid line), $160 \mathrm{~K}$ (dashed line), $77 \mathrm{~K}$ (dotted line) and $4 \mathrm{~K}$ (dash-dot line). For ion radii larger than the thermal equilibrium radius, the distribution depends only on the mass of the buffer gas and not on the gas temperature. Thus, the ion cloud extends to large radius for all gas temperatures.

also be responsible for the observed exponential xenon pressure dependence instead of the simulated power-law dependence. Coulomb interactions have less impact for small numbers of ions, and thus the extrapolation of the simulated fading rate to the observed fading rate is still valid.

\subsubsection{Temperature dependent unloading}

One approach to limit the effect of unloading is to lower the temperature of the buffer gas thereby increasing the buffer gas cooling. We simulated the spatial distribution of ions in 22 mTorr of xenon gas at 4, 77, 160, and $300 \mathrm{~K}$ (corresponding to liquid helium, liquid nitrogen, liquid xenon, and room temperature). The results, plotted in Fig. 7.12, demonstrate that the spatial distribution depends only weakly on the xenon gas temperature. As the temperature changes from $300 \mathrm{~K}$ to $4 \mathrm{~K}$, the fraction of time 
the ion spends at high radius decreases by a factor of $\sim 10$. Lower temperatures change the equilibrium thermal radius as defined by Eq. 7.2 but does not change the fundamental nature of collisions in RF traps. As discussed in $\S 5.5$, when the ion has a higher velocity than the buffer gas, the ion radius changes during each collision as $\partial z \propto z$, independent of the velocity of the buffer gas. For very cold temperatures, the equilibrium ion radius is quite small and so many collisions are required to reach high radii. Once the ion is at a large radius however, the ion extends to even higher radius with exactly the same probability as ions trapped in warm buffer gases. In other words, the diffusive unloading cannot be mitigated by lowering the ion trap temperature. 


\section{Chapter 8}

\section{Conclusion}

In this work, we have applied the techniques of single ion spectroscopy to the developement of a high pressure, single ion detector for use in the Enriched Xenon Observatory neutrinoless double beta decay experiment. We developed a single ion barium spectroscopy cell capable of operation in 100 mtorr of helium buffer gas simultaneously with lower partial pressures of other inert gases. We used this system to systematically examine ion trapping in buffer gases as a function of the buffer gas mass. In addition to confirming the cooling effect of light buffer gases, we characterized the phenomenon diffusive unloading of barium ions by massive buffer gases, particularly as it applies to EXO.

Diffusive unloading, not previously studied in detail, destabilizes a trapped barium ion by extending the spatial distribution of the ion beyond the trapping volume. The ion's spatial extent depends on the mass of the buffer gas; helium $(\mathrm{m}=4)$ and argon $(\mathrm{m}=40)$ do not cause unloading, while krypton $(\mathrm{m}=83)$ and xenon $(\mathrm{m}=136)$ do. As demonstrated with a simple Monte Carlo simulation, collisions between a trapped ion and a buffer gas atom cause a random walk in the maximum ion radius; once the radius extends beyond the trapping volume, the ion unloads. The greater the mass of the buffer gas, the greater the size of each step in the random walk, and the greater the extent of the resulting spatial distribution. Helium gas cools the trapped ion and reduces the diffusive unloading rate. However, both experiment and simulation demonstrate that the unloading persists even with 10 mtorr of helium 
buffer gas and low pressure xenon gas. Diffusive unloading is a general feature of ion collisions in RF traps, and occurs at all of the parameters tested both in simulation and experimentally.

Although diffusive unloading complicates the incorporation of the single ion spectroscopic cell into the full scale EXO detector, several approaches minimize the impact. Most importantly, helium buffer gas cooling extends the lifetime of trapped ions. When the mean time between helium collisions is much shorter than the xenon time, the ion lifetime can be extended to hundreds and even thousands of seconds. We showed experimentally that a ratio of helium to xenon pressure of 10 to 1 is sufficient to extend the trapping time to 1,000 seconds. Although the simulation cannot be used to calculate such long time constants, it does indicate that unloading rates are longer than 10 seconds when the helium pressure is $\sim 100$ times greater than the xenon pressure. The helium pressure dependence sets a design criterion for the EXO barium tagging trap: the helium pressure must be greater than 100 times the xenon pressure during barium ion trapping. To be very conservative, a 1,000 to 1 helium to xenon ratio should ensure that the barium ion does not diffusively unload during the tagging operation.

In conclusion, we have demonstrated single ion trapping and spectroscopy for use in the EXO neutrino-less beta decay experiment. Although diffusive unloading prevents barium ion trapping in a pure xenon atmosphere, high helium partial pressures extend the ion lifetime and stabilize the ion trapping, allowing the incorporation of barium spectroscopy into the EXO detector. With the new tool of final state tagging, the EXO collaboration will achieve unsurpassed sensitivity to the Majorana neutrino mass and provide an invaluable window into physics beyond the standard model. 


\section{Appendix A}

\section{Experimental Setup}

Three major systems are required to trap and observe single barium ions: frequency stabilized lasers, a radio-frequency ion trap, and an ultra-high vacuum system. The construction and operation of each will be discussed in turn.

\section{A.1 Frequency stabilized laser systems}

The three level electronic structure of ionized barium needs two lasers to fluoresce continuously, a blue laser for the $4934 \AA 6 S_{1 / 2}$ to $6 P_{1 / 2}$ transition and a red laser for the $6496 \AA 6 P_{1 / 2}$ to $5 D_{3 / 2}$ transition. In order to maximize the barium ion signal, each laser must have a frequency bandwidth less than the corresponding excited state width, continuous frequency tuning for $>3 \mathrm{GHz}$ around the resonance center, and enough power to saturate the ion, typically $100 \mu \mathrm{W}$ in a $100 \mu \mathrm{m}$ beam. Since the excited state linewidth for the blue transition is $15.2 \mathrm{MHz}$ and the red transition width is $5.1 \mathrm{MHz}$, the laser linewidth requirement is similar for both lasers; the lasers must have a linewidth of $\sim 1 \mathrm{MHz}$. Once transmission losses are taken in to account, each laser must output at least $1 \mathrm{~mW}$ in order to saturate a small number of trapped ions in ultra-high vacuum. 


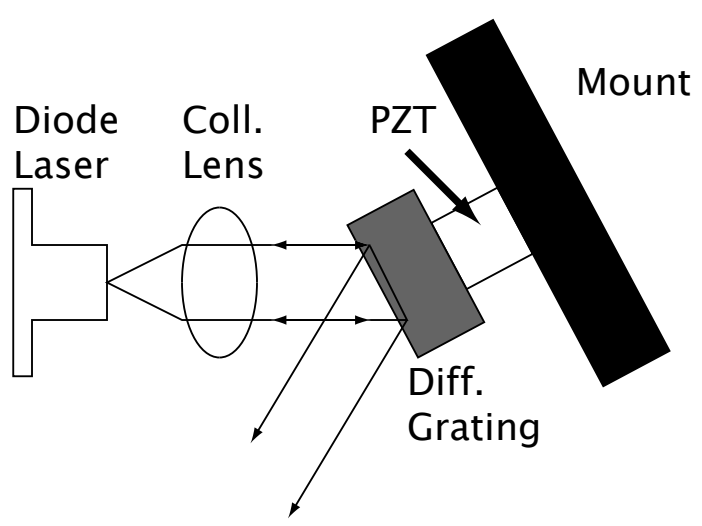

Figure A.1: The External Cavity Diode Laser (ECDL) configuration. The entire assembly is mounted on a TEC for thermal stability. Fine control of the grating position is provided by the piezoelectric transducer (PZT).

\section{A.1.1 External cavity diode lasers}

Both the red and blue lasers use External Cavity Diode Lasers (ECDL) to satisfy the requirements for frequency tuning and stability. This section will provide a brief overview of the lasers used in this experiment; for a more in depth treatment see Yariv[84] or Siegman[85]. In an ECDL, a wide band width semiconductor diode laser provides the lasing medium and the frequency feedback is provided by forming a cavity with an external diffraction grating as shown in Fig. A.1. The overall gain of the ECDL is a function of the semiconductor gain, diode cavity, and external feedback. The frequency bandwidth of an unmodified diode laser is determined only by the gain of the semiconductor materials and the cavity formed by the front and back facets of the diode. Since the diode is physically small, the Free Spectral Range (FSR) of the diode cavity is $c / 2 l \sim 15 \mathrm{GHz}$, where $c$ is the speed of light and $l$ is the optical length of the cavity. Since the reflectivity of the diode facets is relatively low, $\sim 30 \%$, the finesse of the diode cavity is low and does not provide high selectivity or stability. Since the gain of the semiconductor medium is quite broad $\left(\sim 5 \times 10^{5}\right.$ $\mathrm{GHz}$ ), the narrow FSR and low finesse allow the laser to run in multiple longitudinal modes in the absence of external feedback.

To narrow the spectrum and select a single longitudinal mode, the first-order 


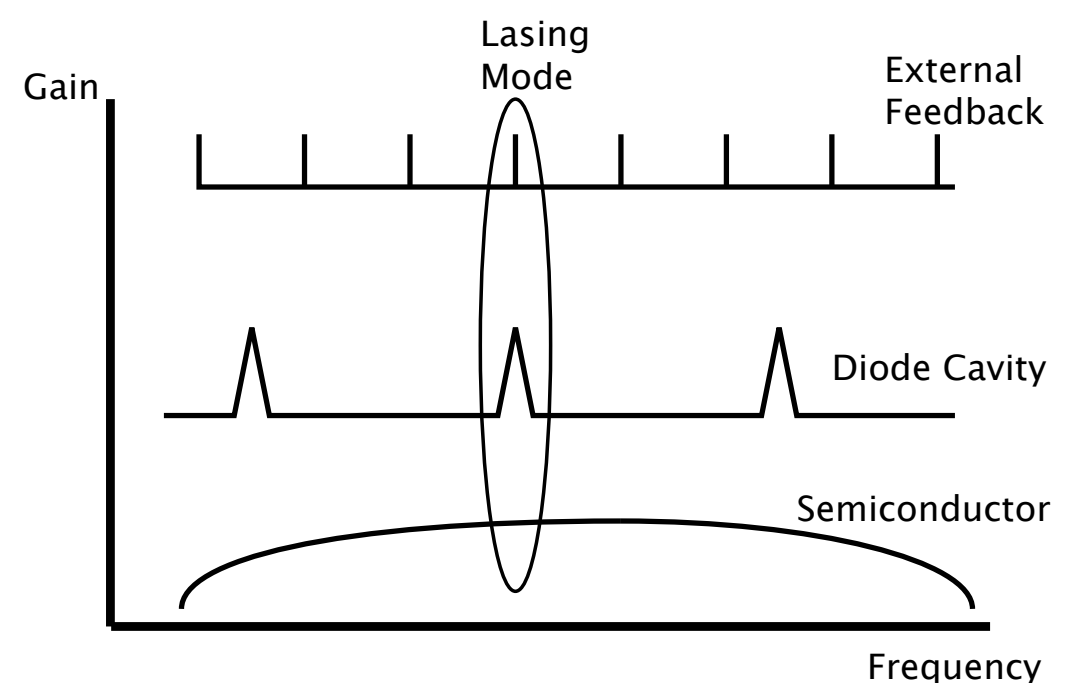

Figure A.2: The gain curves of an external cavity diode laser. The ECDL lases in the mode with the highest gain. The laser frequency can be tuned by changing the length of the external cavity.

diffracted beam from the grating is directed back into the laser diode. The retroreflected electromagnetic wave must satisfy the resonance condition as it travels from the diode laser rear facet to the grating and back, forming a standing wave with FSR of $c / 2 l \sim .5 \mathrm{GHz}$. The narrow FSR of the external cavity provides an additional gain which selects a single longitudinal diode mode. As the net gain of the ECDL is the product of each independent factor, the laser will only lase in the mode with the highest gain. The grating angle and length of the external cavity are tuned by a piezoelectric transducer (PZT) mounted on the grating and used to tune the laser frequency. The effective gains for an ECDL are drawn schematically (and not to scale) in Fig. A.2. A mode-hop-free scanning range of $3 \mathrm{GHz}$ is easily obtained by scanning the PZT alone. In order to achieve higher scanning ranges, the current to the laser diode must be tuned along with the PZT voltage in order to tune the cavity modes in step with the external modes. 


\section{A.1.2 Second harmonic generation}

At the time of the writing of this thesis, no commercially available narrowband laser directly outputs light at $4934 \AA$. Consequently, the light must be created by Second Harmonic Generation (SHG) of the output of a $9860 \AA$ ECDL. The $4930 \AA$ laser system used in this work is a Toptica Photonics SHG-100 capable of providing 20 $\mathrm{mW}$ of blue output. The layout of the SHG-100, as shown in Figure A.3, consists of a $9860 \AA$ ECDL followed by an optical isolator and a birefringent $\mathrm{KNbO}_{3}$ crystal in a doubling cavity. SHG takes advantage of the large non-linear index of refraction, $n(I)=n_{0}+n_{1} I+n_{2} I^{2} \ldots$ of $\mathrm{KNbO}_{3}$ to up-convert two photons of long wavelength into one photon of short wavelength. For SHG to proceed efficiently, the short and long wavelength photons must remain in phase as they travel through the crystal. At a temperature of $33 \mathrm{C}$, the $\mathrm{KNbO}_{3}$ crystal's birefringence matches the phase velocities of the $4930 \AA$ and $9860 \AA$ photons as they propagate along orthogonal crystal axes, allowing efficient conversion.

\section{A.1.3 Frequency stabilization}

We use a Burleigh WA-1000 wavemeter to set the laser frequency of both the red and blue lasers to $\pm 500 \mathrm{MHz}$. Although the precision of the wavemeter is $\pm 100 \mathrm{MHz}$, the helium-neon reference laser in the wavemeter drifts with temperature, limiting the measurement accuracy to the quoted value. The $500 \mathrm{MHz}$ accuracy of the wavemeter is sufficient to acquire an ion signal from which a more accurate frequency determination can be made. For short term stability, both lasers are locked to confocal Invar cavities with a FSR of $750 \mathrm{MHz}$ using a Pound-Drever locking scheme. In order to reduce the sensitivity of the reference cavities to environmental change, they are enclosed in an air-tight, heavy-walled aluminum box. The box rests on sorbothane pads as do the cavities within the box in order to decouple the cavities from table vibrations. Although cavity stabilization greatly improves the laser stability for times shorter than $\sim 100$ minutes, the laser frequencies drift over the course of several hours. In order to perform very long measurements in the future, the lasers will be locked to a temperature controlled zerodur cavity. 


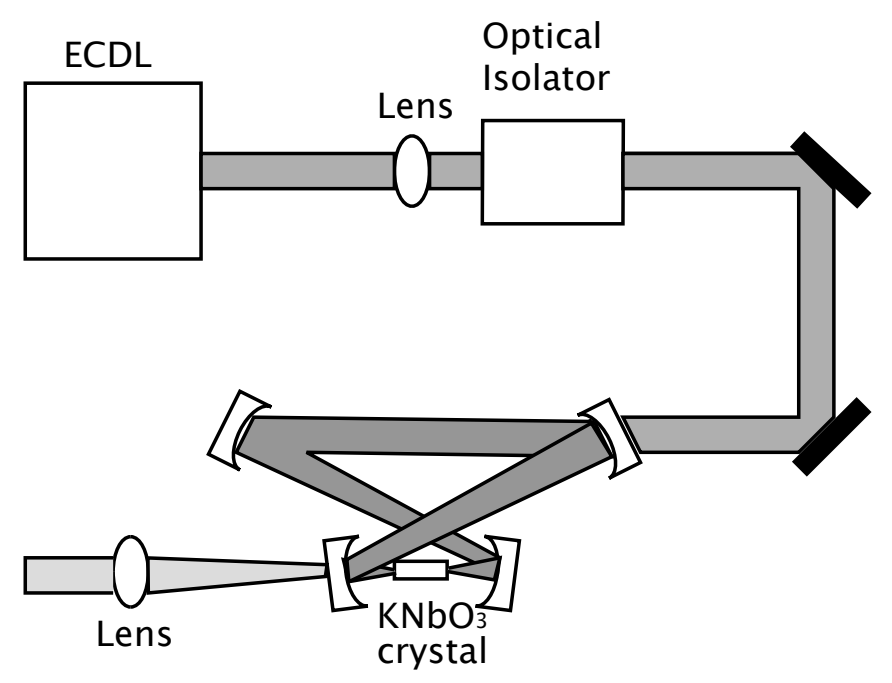

Figure A.3: Schematic layout of the Second Harmonic Generation cavity used to make the $4930 \AA$ laser wavelength. The $\mathrm{KNbO}_{3}$ crystal is temperature controlled, and the SHG ring cavity is locked to the ECDL wavelength. 


\section{A.2 Vacuum and gas handling}

Trapping ions in Ultra-High Vacuum (UHV) conditions requires a vacuum pressure better than $1 \times 10^{-9}$ torr. At higher pressures, collisions with the background molecules overwhelms the laser cooling and the ion either unloads or leaves the laser beams and cannot be seen. In practice, our system is capable of attaining an ultimate base pressure of $P \leq 2 \times 10^{-10}$ torr. A schematic of the vacuum and gas manifolds is shown in Fig. A.4. In order to achieve these low pressures, the vacuum system is made of Con-Flat UHV hardware and the gas manifold consists of a mixture of Con-Flat and metal gasket VCR fittings. A $55 \mathrm{l} / \mathrm{s}$ Starcell ion pump evacuates the vacuum tank, while oil-free roughing and turbo pumps are used for the initial pump down. All valves have metal seats and bonnet seals. The mechanical fittings inside the vacuum system are made of copper, stainless steel, Macor, and nickel in order to maintain the lowest base pressure, and all vacuum parts are ultrasonically cleaned in detergent, acetone, and ethanol before installation. Custom, in-situ ovens are used to bakeout the gas manifold and vacuum system to $150 \mathrm{C}$ for several days after exposure to air in order to remove adsorbed water and gas from the vacuum surfaces. The ovens greatly decrease the time and effort required to cycle the vacuum chamber from atmosphere to UHV, minimizing the cost in time and effort required to work on the ion trap internals. We cannot stress enough how important the ovens have been in minimizing the experiment turnaround and maximizing the experiments potential.

We have studied Ba-II trapping with helium, nitrogen, argon, krypton and xenon. The highly reactive Ba-II ions form chemical compounds with most other gas species such as oxygen, carbon monoxide, and carbon dioxide. In order to eliminate these trace impurities from the inert buffer gases, we flow the gas through a SAES hot zirconium getter before introducing the gas to the vacuum chamber. The pressure in the gas manifold is controlled using a mechanical 30/30 gauge and standard gas regulators. Both a cold-cathode magnetron gauge and an extended range Pirani gauge monitor the pressure in the vacuum tank. Depending on the gas used, the gauges provide coverage from $1 \times 10^{-10}$ torr to 1 atm, with a small gap at $1 \times 10^{-3}$ torr 


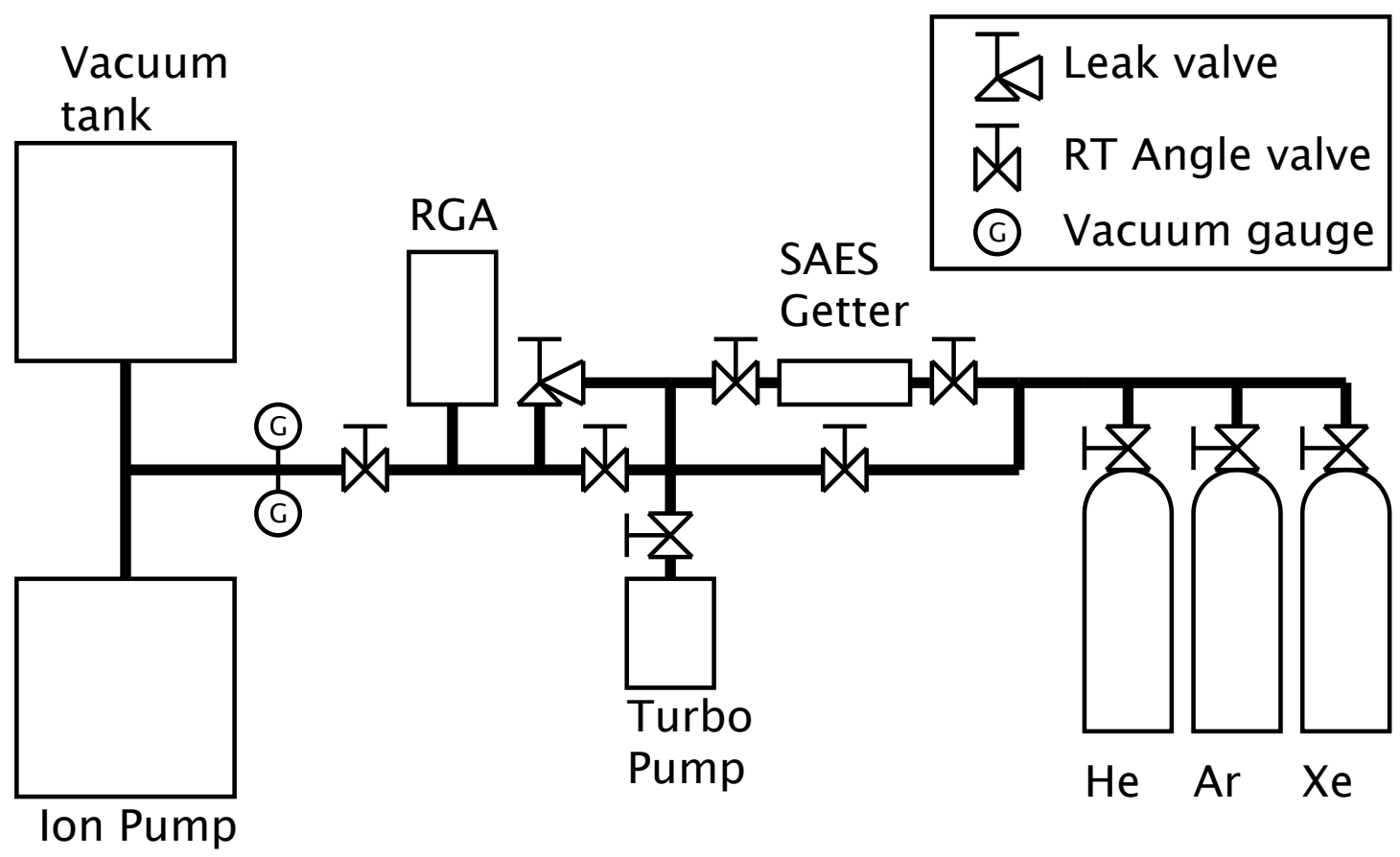

Figure A.4: Layout of the vacuum and gas handling systems. The ion pump, turbo pump, and leak valve control the pressure from $<2 \times 10^{-10}$ to 0.1 torr. Helium, argon, krypton, and xenon have been used. 
where the gauges do not overlap. Although thermocouple vacuum gauges such as the Pirani gauge have poor sensitivity to xenon pressure above $1 \times 10^{-2}$ torr, it was not an issue in this work. A Varian-style, sapphire seat leak valve controls the gas feed rate into the vacuum. In order to avoid distorting the ion trapping measurements, the ion pump is turned off before gas is introduced to the vacuum tank. Due to the large amounts of helium and argon the ion pump has pumped in the past, the vapor pressure in the vacuum system naturally rises to $\sim 1 \times 10^{-5}$ torr when the pump is turned off. A Stanford Research Systems RGA-300 residual gas analyzer provides leak checking and gas monitoring capabilities to ensure good vacuum and gas purity.

\section{A.3 RF quadrupole trap electronics}

A schematic of the RF electronics is shown in Fig. A.5. The RF quadrupole ion trap used in this work has a $15 \mathrm{pF}$ capacitance operating at $3000 V_{p p}$ and $8.5 \mathrm{MHz}$. The vacuum feedthru and wiring layout increase the trap capacitance to $\sim 25 \mathrm{pF}$, equivalent to $750 \Omega$ at the operating frequency. To avoid dissipating the $1.3 \mathrm{~kW}$ required to drive the $\mathrm{RF}$ trap directly, we configure the trap as the capacitive element in an $\mathrm{LC}$ resonator. Because of the low capacitance of the trap, the inductor is quite large, $\sim 10 \mu F$. Great care is taken to maximize the inductor quality factor (Q) so that the input power is minimized; $Q \sim 50$ was used for this work and was achieved with a 2-inch air-core toroid wrapped with 44 turns of 26 gage magnet wire. The RF signal is generated in a Hewlett-Packard function generator and amplified by a broadband, $100 \mathrm{~W}$ RF amplifier with $50 \Omega$ output impedance. A $\pi$-network impedance matches the RF amplifier to the LC-resonator and a small, variable capacitor in parallel to the trap capacitance allows the resonance frequency to be tuned from 6.8 to $9.8 \mathrm{MHz}$. The trap voltage is continuously monitored using a $0.3 \mathrm{pF}$ capacitive pick-off and divider. All of the components with high voltage RF are enclosed in a single heavy walled aluminum box mounted directly to the vacuum system in order to minimize RF pick-up in the laboratory. 


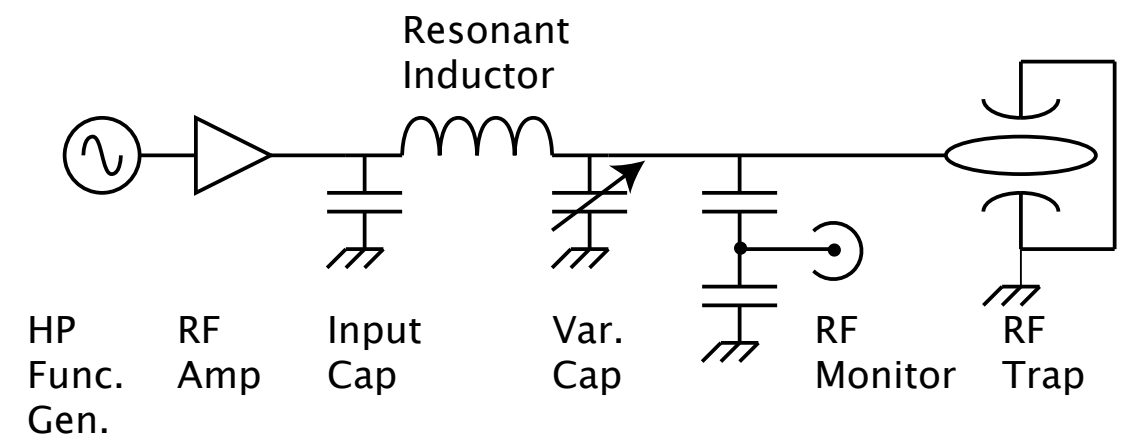

Figure A.5: Schematic of the RF electronics. The $3000 V_{p k p k}$ is applied to the ring via a function generator, $\mathrm{RF}$ amplifier, impedance matching $\pi$-network, and resonant LC circuit. The resonant frequency is controlled with a small variable capacitor and the $\mathrm{RF}$ voltage is monitored with a capacitive pickoff and divider. 


\section{Bibliography}

[1] Y. Ashie and et. al. Evidence for an oscillatory signature in atmospheric neutrino oscillations. Phys. Rev. Let., 93(10):101801/1-6, 2004.

[2] S. N. Ahmed and et.al. Measurement of the total active ${ }^{8} \mathrm{~B}$ solar neutrino flux at the sudbury neutrino observatory with endhanced neutral current sensitivity. Phys. Rev. Let., 92(18):181301/1-6, 2004.

[3] K. Eguchi and et. al. First results from KamLAND: evidence for reactor antineutrino disappearance. Phys. Rev. Lett., 90(2):021802/1-6, 2003.

[4] H.V. Klapdor-Kleingrothaus and et.al. Latest results from the HEIDELBERGMOSCOW double beta decay experiment. Eur. Phys. J. A, 12:147-154, 2001.

[5] M. K. Moe. New approach to the detection of neutrinoless double-beta dcay. Phys. Rev. C, (44):931-934, 1991.

[6] W. Neuhauser, M. Hohenstatt, P. E. Toschek, and H. Dehmelt. Localized visible $\mathrm{Ba}^{+}$mono-ion oscillator. Phys. Rev. A, 22:1137-1140, 1980.

[7] V. Barger and K. Whisnat. Majorana neutrino masses from neutrinoless double beta decay and cosmology. Phys. Lett. B, 456:194, 1999.

[8] J. Ellis and S. Lola. Can neutrinos be degenerate in mass? Phys. Lett. B, 458:310, 1999.

[9] D. O. Caldwell and R. N. Mohapatra. Neutrino mass explanations of solar and atmospheric neutrino deficits and hot dark matter. Phys. Rev. D, 48:3259, 1993. 
[10] S. M. Bilenki and et.al. Constraints from neutrino oscillation experiments on the effective majorana mass in neutrinoless double $\beta$-decay. Phys. Lett. B., 465:193, 1999.

[11] C. Weinheimer. Direct neutrino mass search. arXiv:hep-ex, (0210050v2), 2002.

[12] C. Weinheimer. KATRIN, a next generation tritium beta decay experiment in search for the absolute neutrino mass scale. Prog. Part. Nucl. Phys., (48):141$150,2002$.

[13] M. Moe. The status of two-neutrino and neutrinoless double beta decay searches. Int. J. Mod. Phys., E2:507, 1993.

[14] M. Doi, T. Kotani, and E. Takasugi. Double beta decay and Majorana neutrino. Prog. Theor. Phys., 83 (Suppl.):1, 1985.

[15] M. Moe and P. Vogel. Double beta decay. Ann. Rev. Nucl. Part. Sci., 44:247, 1994.

[16] R. D. McKeown and P. Vogel. Neutrino masses and oscillations: Triumphs and challenges. arXiv:hep-ph, (0402025), 2004.

[17] F. Boehm and P. Vogel. Physics of Massive Neutrinos. Cambridge University Press, 2 edition, 1992.

[18] C. E. Aalseth and et. al. Comment on "Evidence for neutrinoless double beta decay". Mod. Phys. Lett., A, 2002.

[19] M. Hirsch and H. V. Klapdor-Kleingrothaus. Some particle physics aspects of neutrinoless double beta decay. Prog. Part. Nucl. Phys., 40:323, 1998.

[20] M. Hirsch, H. V. Klapdor-Kleingrothaus, S. Kolb, and S. G. Kovalenko. Phenomenological implications of "Majorana" sneutrinos at future accelerators. Phys. Rev. D, 57:2020, 1998.

[21] E. Caurier, F. Nowacki, A. Poves, and J. Retamosa. Shell model study of the neutrinoless double beta decays. Nucl. Phys. A, 654:973c, 1999. 
[22] V. A. Rodin, A. Faessler, F. Simkovic, and P. Vogel. Uncertainty in the $0 \nu \beta \beta$ decay nuclear matrix elements. Phys. Rev. C, 68:0044302, 2003.

[23] K. You and et.al. A search for neutrinoless double $\beta$ decay of ${ }^{48}$ Ca. Physics Letters B, 265:53-56, 1991.

[24] X. Sarazin and et.al. Search for neutrinoless double beta decay with the NEMO-3 detector: first results. arXiv:hep-ex, (0412012v1), 2004.

[25] F. A. Danevich and et.al. Search for $2 \beta$ decay of cadmium and tungsten isotopes: Final results of the Solotvina experiment. Phys. Rev. C, 68:035501, 2003.

[26] C. Arnaboldi and et.al. A new limit on the neutrinoless $\beta \beta$-decay of ${ }^{130} \mathrm{Te}$. arXiv:hep-ex, (0501034), 2005.

[27] R. Bernabei and et. al. Investigation of $\beta \beta$ decay modes in ${ }^{134} \mathrm{Xe}$ and ${ }^{136} \mathrm{Xe}$. Phys. Let. B, 546:23-28, 2002.

[28] A. De Silva and et. al. Double $\beta$ decays of ${ }^{100} \mathrm{Mo}$ and ${ }^{150} \mathrm{Nd}$. Phys. Rev. C, 56:2451-2467, 1997.

[29] A. Staudt and et. al. Calculation of $2 \mathrm{nu}$ and $0 \mathrm{nu}$ double-beta decay rates. Europhys. Lett., 13:31, 1990.

[30] A. Staudt and et.al. Nuclear matrix elements for double positron emission. Phys. Lett. B, 268:312, 1991.

[31] E. Caurier and et.al. Shell model studies of the double beta decays of sup 76Ge, sup 82Se, and sup 136Xe. Phys. Rev. Lett., 77:1954, 1996.

[32] H. V. Klapdor-Kleingrothaus and et.al. Search for neutrinoless double beta decay with enriched 76Ge in Gran Sasso 1990-2003. Phys. Lett. B, 586:400, 2004.

[33] C. Arnaboldi and et. al. First results on neutrinoless double beta decay of ${ }^{130} \mathrm{Te}$ with the calorimetric CUORICINO experiment. Phys Lett B, 256:260, 2004. 
[34] R. Luescher and et. al. Search for $\beta \beta$ decay in ${ }^{136} \mathrm{Xe}$ : new results from the gotthard experiment. Phys. Lett. B, 434:407, 1998.

[35] Esch and Hime. private communication, 2000.

[36] E. Conti and et. al. Correlated fluctuations between luminescence and ionization in liquid xenon. Phys. Rev. B, 68:054201, 2003.

[37] J. Engel, P. Vogel, and M. R. Zirnbauer. Nuclear structure effects in double-beta decay. Phys. Rev. C, 37:731, 1988.

[38] R. L. Liboff. Introductory Quantum Mechanics. Addison-Wesley, 1991.

[39] J. J. Sakurai. Modern Quantum Mechanics. Addison-Wesley, 1994.

[40] A. Yariv. Quantum Electronics. John Wiley \& Sons, 1989.

[41] O. Svelto. Principles of Lasers. Plenum Press, 1989.

[42] R. D. Cowan. The Theory of Atomic Structure and Spectra. University of California Press, 1981.

[43] C. Cohen-Tannoudji, J. Dupont-Roc, and G. Grynberg. Atom-Photon Interactions. John Wiley \& Sons, 1992.

[44] G. R. Janik. Laser cooled single ion spectroscopy of magnesium and barium. $\mathrm{PhD}$ thesis, University of Washington, 1984.

[45] N. Yu, W. Nagourney, and H. Dehmelt. Radiative lifetime measurement of the $\mathrm{Ba}^{+}$metastable $\mathrm{D}_{3 / 2}$ state. Phys. Rev. Let., 78:4898-4901, 1997.

[46] D. Bellert and W. H. Breckenridge. Bonding in ground-sate and excited state $\mathrm{a}^{+} \cdot \mathrm{rg}$ van der waals ions $(\mathrm{a}=$ atom, $\mathrm{rg}=$ rare-gas atom): A model-potential analysis. Chem. Rev., 102:1595-1622, 2002.

[47] C. J. Kleinman, Y. Hahn, and L. Spruch. Dominant nonadiabatic contribution to the long-range electron-atom interaction. Phys. Rev., 165(1):53-62, 1968. 
[48] W. Demtroder. Laser Spectroscopy. Springer, 2003.

[49] E. Ehrlacher and J. Huennekens. Noble-gas broadening rates for the $6 s^{21} s_{0} \rightarrow$ $6 s 6 p^{13} p_{1}$ resonance and intercombination lines of barium. Phys. Rev. A, 47(4):3097 - 3104, 1993.

[50] A. Gallagher. Oscillator strengths of Ca II, Sr II, and Ba II. Phys. Rev., 157:24, 1967.

[51] R. Blümel, C. Kappler, W. Quint, and H. Wlather. Chaos and order of lasercooled ions in a Paul trap. Phys. Rev. A, 40(2):808-823, 1989.

[52] W. Paul. Electromagnetic traps for charged and neutral particles. Reviews of Modern Physics, 62(3):531-540, July 1990.

[53] W. Neuhauser, M. Hohenstatt, P. E. Toschek, and H. Dehmelt. Localized visible $\mathrm{Ba}^{+}$mono-ion oscillator. Physical Review A, 22(3):1137-1140, September 1980.

[54] R. F. Wuerker, H. Shelton, and R. V. Langmuir. Electrodynamic containment of charged particles. Journal of Applied Phyiscs, 30(3):342-349, March 1959.

[55] N. W. McLachlan. Theory and Application of Mathieu Functions. Dover Publications, 1964.

[56] H. G. Dehmelt. Radiofrequency spectroscopy of stored ions I: Storage. Adv. At. Mol. Phys., 3:53-72, 1967.

[57] L.D. Landau and Lifschitz. Mechanics. Pergman, Oxford, 1960.

[58] N. Yu, W. Nagourney, and H. Dehmelt. Demonstration of new Paul-Strauble trap for trapping single ions. J. Appl. Phys., 69:3779, 1990.

[59] J. D. Jackson. Classical Electrodynamics. John Wiley and Sons, 1999.

[60] Kotowski. Z. Angew. Math., Mech., 23:213, 1943.

[61] Y. Wang, J. Franzen, and K. P. Wanczek. The non-linear resonance ion trap. 2. Int. J. Mass Spec. and Ion Proc., 124(2):125-44, 1993. 
[62] R. Alheit, X. Z. Chu, M. Hoefer, M. Holzki, and G. Werth. Nonlinear collective oscillations of an ion cloud in a Paul trap. Phys. Rev. A, 56:4023, 1997.

[63] L. S. Cutler, R. P. Giffard, and M. D. McGuire. Thermalization of ${ }^{199} \mathrm{Hg}$ ion macromotion by a light background gas in an RF quadrupole trap. Appl. Phys. $B, 36: 137,1985$.

[64] F. G. Major, V. N. Gheorghe, and G. Werth. Charged particle traps. Springer, 2005.

[65] H. Straubel. Kurze originalmitteilungen. Die Naturwissenscahten, 42:506-507, 1955.

[66] J. S. Brodbelt. Effects of collisional cooling on ion detection. In R. E. March and J. F. J. Todd, editors, Practical aspects of Ion trap mass spectrometry, volume 1, pages 209-220. CRC Press, 1995.

[67] T. Hasegawa and K. Uehara. Dynamics of a single particle in a Paul trap in the presence of the damping force. Appl. Phys. B, 61:159-163, 1995.

[68] J. V. José and E. J. Saletan. Classical Dynamics: A contemporary approach. Cambridge University Press, 1998.

[69] M. Nasse and C. Foote. Influence of background pressure on the stability region of a Paul trap. Eur. J. Phys., 22:563-573, 2001.

[70] M. D. N. Lunney, F. Buchinger, and R. B. Moore. The temperature of buffer-gas cooled ions in a Paul trap. Journal of Modern Optics, 39:349-360, 1992.

[71] R. D. Smith A. V. Tolmachev, H. R. Udseth. Modeling the ion density distribution in collisional cooling RF multipole ion guides. Int. J. Mass Spec., 222:155-174, 2003.

[72] F. A. Londry, R. L. Alfred, and R. E. March. Computer simulation of single-ion trajectories in Paul-type ion traps. J. Am. Soc. Mass Spectrrometry, 4:687-705, 1993. 
[73] Y. Moriwaki and T. Shimizu. Effect of a heavy collision partner on ion loss from a radio frequency trap. Jpn. J. Appl. Phys., 37:334-348, 1998.

[74] J. Hoffnagle, R. G. DeVoe, L. Reyna, and R. G. Brewer. Order-chaos transition of two trapped ions. Phys. Rev. Lett., 61:255-258, 1988.

[75] R. Blümel, J. M. Chen, E. Peik, W. Quint, W. Schleich, Y. R. Shen, and H. Walther. Phase transitions of stored laser-cooled ions. Nature, 334:309-313, 1988.

[76] E. W. McDaniel. Atomic Collisions. John Wiley \& Sons, 1989.

[77] R. Blatt, P. Zoller, G. Holzmüller, and I. Siemers. Brownian motion of a parameteric oscillator: A model for ion confinement in radio frequency traps. Z. Phys. D, 4:121-126, 1986.

[78] N. G. Van Kampen. Stochastic processes in physics and chemistry. Elsevier Science, B.V., 1992.

[79] M. C. Wang and G. E. Uhlenbeck. On the theory of the Brownian motion II. Rev. Mod. Phys., 17:323-342, 1945.

[80] R. E. Johnson. Introduction to Atomic and Molecular Collisions. Plenum Press, 1982.

[81] P. Langevin. Ann. Chim. Phys. Ser., 8(5):245-288, 1905.

[82] Numerical Recipes in C. Cambridge University Press, 2 edition, 1992.

[83] Statistics. Physics Letters B, page 287, 2004.

[84] A. Yariv. Quantum Electronics. John Wiley \& Sons, 3 edition, 1989.

[85] A. E. Siegman. Lasers. University Science Books, 1986. 